\title{
Synthesis and reactivity of spirocarbocycles as scaffolds for nucleoside analogues
}

Jonas Verhoeven, ${ }^{*[a-b]}$ Xavier Deraet, ${ }^{[a]}$ Vineet Pande, ${ }^{[b]}$ Weimei Sun, ${ }^{[b]}$ Mercedes Alonso, ${ }^{[a]}$ Frank De Proft, ${ }^{[a]}$ Lieven Meerpoel, ${ }^{[b]}$ Jan Willem Thuring ${ }^{[b]}$ and Guido Verniest ${ }^{[a-b]}$

[a] J. Verhoeven, G. Verniest, Research Group of Organic Chemistry (ORGC), Department of Chemistry and Department of Bio-engineering Sciences, Faculty of Science and Bio-engineering Sciences, Vrije Universiteit Brussel (VUB), Pleinlaan 2, 1050 Brussels (Belgium) E-mail: jverhoe9@its.jnj.com

X. Deraet, M. Alonso, Prof. F. De Proft, Research Group of General Chemistry (ALGC), Vrije Universiteit Brussel (VUB), Pleinlaan 2, 1050 Brussels (Belgium)

[b] J. Verhoeven, V. Pande, L. Meerpoel, J. W. Thuring, G. Verniest, Janssen Pharmaceutica NV, Turnhoutseweg 30, 2340 Beerse (Belgium)

\section{Table of Contents}

Mass Chromatography - Method tables

Computational DFT calculations

Ketoreductase screening

In-vitro inhibition studies

Copies of ${ }^{1} \mathrm{H}$ and ${ }^{13} \mathrm{C}$ NMR spectra of all new compounds 
SUPPORTING INFORMATION

Mass Chromatography - Method tables

S2

\begin{tabular}{|c|c|c|c|c|c|c|c|}
\hline $\begin{array}{l}\text { Method } \\
\text { number }\end{array}$ & Instrument & Column & Mobile phase & Gradient & Flow & $\begin{array}{c}\text { Column } \\
\text { temp. }\end{array}$ & $\begin{array}{l}\text { Run } \\
\text { time }\end{array}$ \\
\hline 1 & $\begin{array}{l}\text { Waters: } \\
\text { Breeze } \\
\text { HPLC - } 2489 \\
\text { UVD }\end{array}$ & $\begin{array}{l}\text { YMC C18 }(5.0 \\
\mu m, \quad 4.6^{\star} 250 \\
\mathrm{~mm})\end{array}$ & $\begin{array}{l}\mathrm{A}: \mathrm{CH}_{3} \mathrm{CN} \\
+0.1 \% \text { TFA } \\
\text { B: } \mathrm{H}_{2} \mathrm{O}+0.1 \% \\
\text { TFA }(v / v)\end{array}$ & $\begin{array}{l}\text { From } 3 \% A \text { to } \\
97 \% A \text { in } 20 \mathrm{~min} .\end{array}$ & 0.3 & 25 & 21 \\
\hline 2 & $\begin{array}{l}\text { Waters: } \\
\text { Acquity }^{\circledR} \\
\text { UPLC - DAD } \\
\text { and SQD }\end{array}$ & $\begin{array}{l}\text { Waters: BEH } \\
\text { C18 }(1.7 \mu \mathrm{m}, \\
\left.2.1^{*} 50 \mathrm{~mm}\right)\end{array}$ & $\begin{array}{l}\mathrm{A}: 10 \mathrm{mM} \\
\mathrm{CH} 3 \mathrm{COONH} 4 \\
\text { in } 95 \% \mathrm{H}_{2} \mathrm{O}+ \\
5 \% \mathrm{CH} 3 \mathrm{CN} \\
\text { B: } \mathrm{CH} 3 \mathrm{CN}\end{array}$ & $\begin{array}{l}\text { From } 95 \% A \text { to } \\
5 \% A \text { in } 1.3 \mathrm{~min} \text {, } \\
\text { held for } 0.7 \mathrm{~min} \text {. }\end{array}$ & 0.8 & 55 & 2 \\
\hline 3 & $\begin{array}{l}\text { Waters: } \\
\text { Acquity }^{\circledR} \\
\text { UPLC - DAD } \\
\text { and SQD }\end{array}$ & $\begin{array}{l}\text { Waters: HSS } \\
\text { T3 (1.8 } \mu \mathrm{m} \text {, } \\
\left.2.1^{*} 100 \mathrm{~mm}\right)\end{array}$ & $\begin{array}{l}\mathrm{A}: 10 \mathrm{mM} \\
\mathrm{CH} \mathrm{COONH}_{4} \\
\text { in } 95 \% \mathrm{H}_{2} \mathrm{O}+ \\
5 \% \mathrm{CH}_{3} \mathrm{CN} \\
\mathrm{B}: \mathrm{CH}_{3} \mathrm{CN}\end{array}$ & $\begin{array}{l}\text { From } 100 \% A \text { to } \\
5 \% A \text { in } 2.10 \mathrm{~min} \text {, } \\
\text { to } 0 \% A \text { in } 0.90 \\
\text { min, to } 5 \% A \text { in } \\
0.5 \mathrm{~min}\end{array}$ & 0.7 & 55 & 3.5 \\
\hline 4 & $\begin{array}{l}\text { Waters: } \\
\text { Acquity }^{\circledR} \\
\text { UPLC - DAD } \\
\text { and SQD }\end{array}$ & $\begin{array}{l}\text { Waters: BEH } \\
\text { C18 }(1.8 \mu \mathrm{m}, \\
\left.2.1^{*} 100 \mathrm{~mm}\right)\end{array}$ & $\begin{array}{l}\mathrm{A}: 10 \mathrm{mM} \\
\mathrm{CH} 3 \mathrm{COONH} 4 \\
\text { in } 95 \% \mathrm{H}_{2} \mathrm{O}+ \\
5 \% \mathrm{CH} 3 \mathrm{CN} \\
\mathrm{B}: \mathrm{CH}_{3} \mathrm{CN}\end{array}$ & $\begin{array}{l}\text { From } 100 \% A \text { to } \\
5 \% A \text { in } 2.10 \mathrm{~min} \text {, } \\
\text { to } 0 \% A \text { in } 0.90 \\
\text { min, to } 5 \% A \text { in } \\
0.5 \text { min }\end{array}$ & 0.7 & 55 & 3.5 \\
\hline
\end{tabular}

Table S1. Description of analytical LC-MS method, flow expressed in $\mathrm{ml} / \mathrm{min}$; column temperature $(\mathrm{T})$ in ${ }^{\circ} \mathrm{C}$; Run time in minutes.

\begin{tabular}{|c|c|c|c|c|c|c|c|}
\hline $\begin{array}{l}\text { Method } \\
\text { number }\end{array}$ & Column & $\begin{array}{l}\text { Mobile } \\
\text { phase }\end{array}$ & Gradient & Flow & $\begin{array}{l}\text { Column } \\
\text { temp. }\end{array}$ & Run time & BPR \\
\hline 1 & 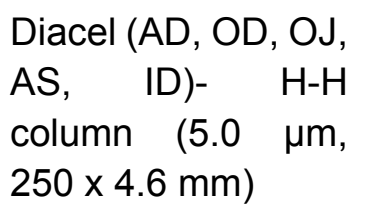 & $\begin{array}{l}\mathrm{A}: \mathrm{CO}_{2} \\
\mathrm{~B}: \mathrm{EtOH}\end{array}$ & $\begin{array}{l}10 \%-55 \% \mathrm{~B} \text { in } 4 \\
\mathrm{~min}, 55-50 \% \text { in } \\
0.45 \mathrm{~min} \text { hold } \\
2.55 \mathrm{~min}\end{array}$ & 5 & 40 & 7 & 110 \\
\hline
\end{tabular}

Table S2. Description of analytical SFC-MS method, flow expressed in $\mathrm{ml} / \mathrm{min}$; column temperature $(\mathrm{T})$ in ${ }^{\circ} \mathrm{C}$; Run time in minutes; Backpressure (BPR) in bars. 


\section{Computational DFT calculations}

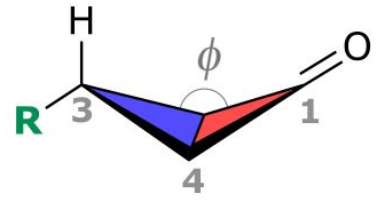

Puckering angle
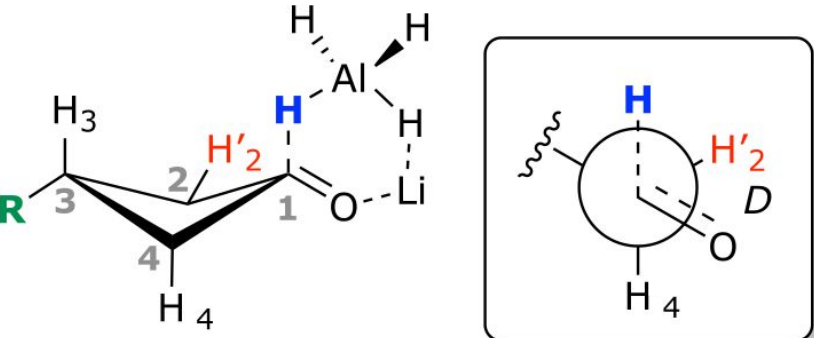

$D\left(\mathrm{O}-\mathrm{C}_{1}-\mathrm{C}_{2}-\mathrm{H}_{2}^{\prime}\right)$

Figure S1. Schematic representation of the puckering angle $\phi$ and dihedral angle $D$ used to establish the influence of torsional strain and eclipsed interactions on the observed stereoselectivity of the reduction reactions. Figure taken from reference 15 in the publication.
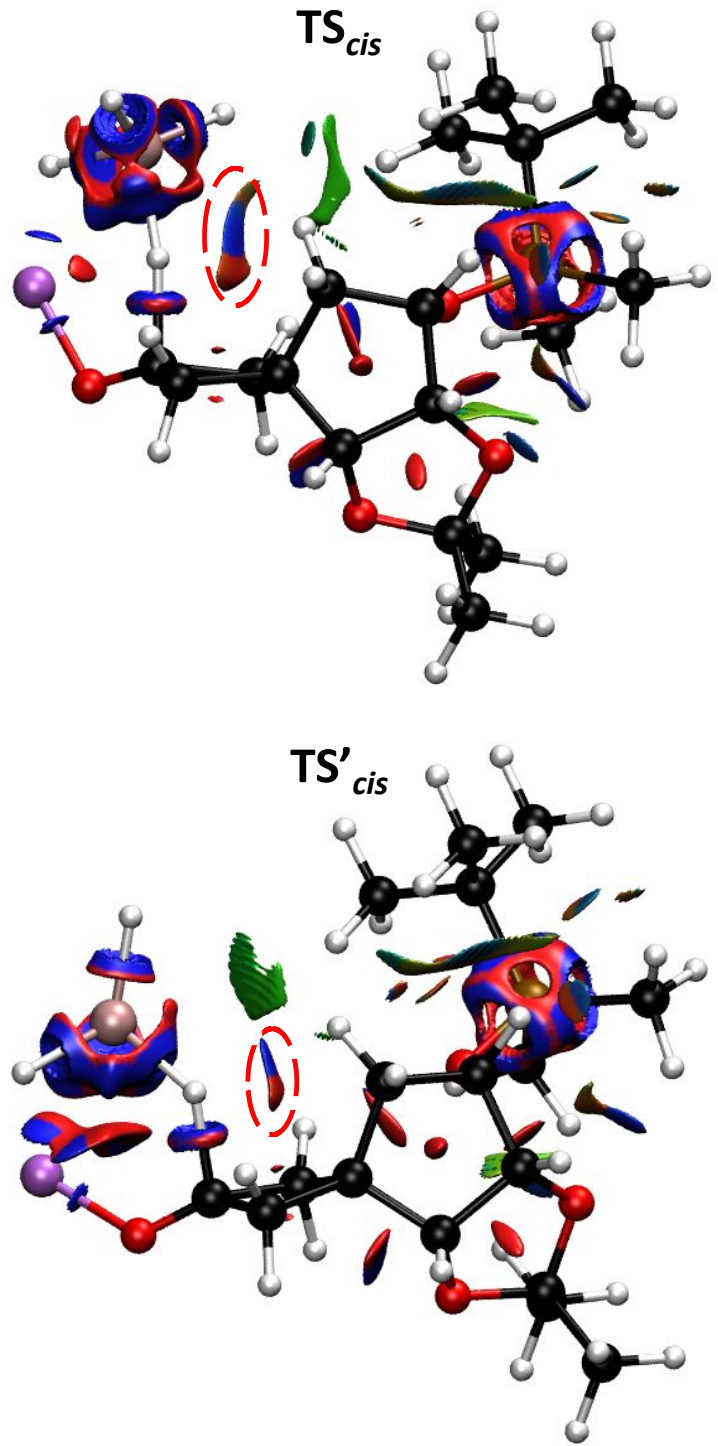

TS

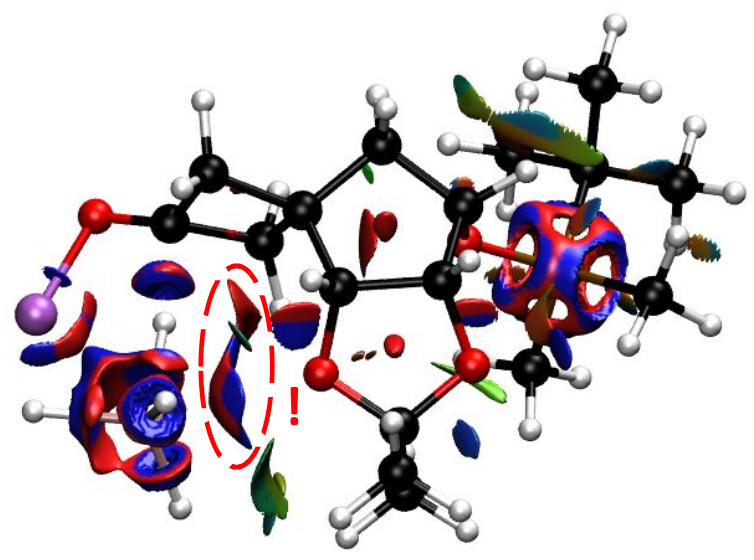

$\mathrm{TS}_{\text {trans }}$

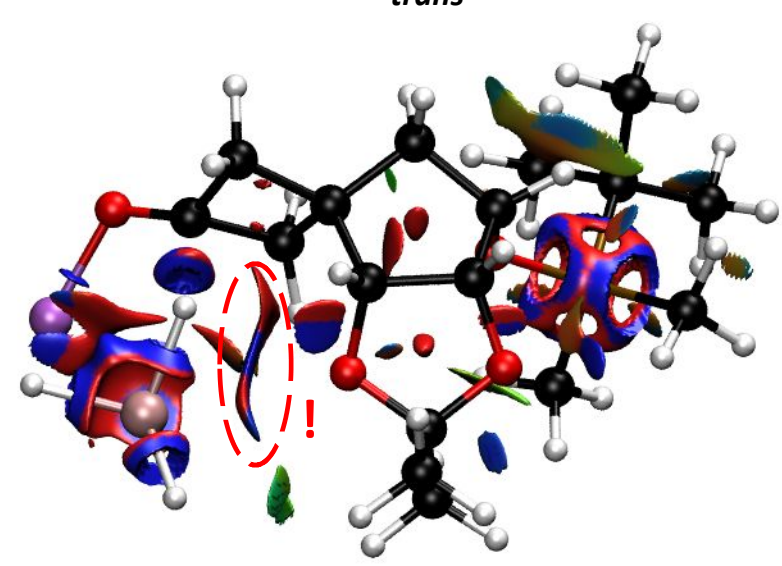

Figure S2. Gradient isosurfaces (isovalue $=0.45$ ) representing the non-covalent interactions occurring in the various transition states describing the reduction of compound 17 with $\mathrm{LiAlH}_{4}$. These isosurfaces are coloured on a RGB scale according to sign $\left(\lambda_{2}\right) \rho$ over the range -0.04 a.u. to 0.04 a.u. The red circle marks the repulsive interactions of interest for the observed selectivity. 

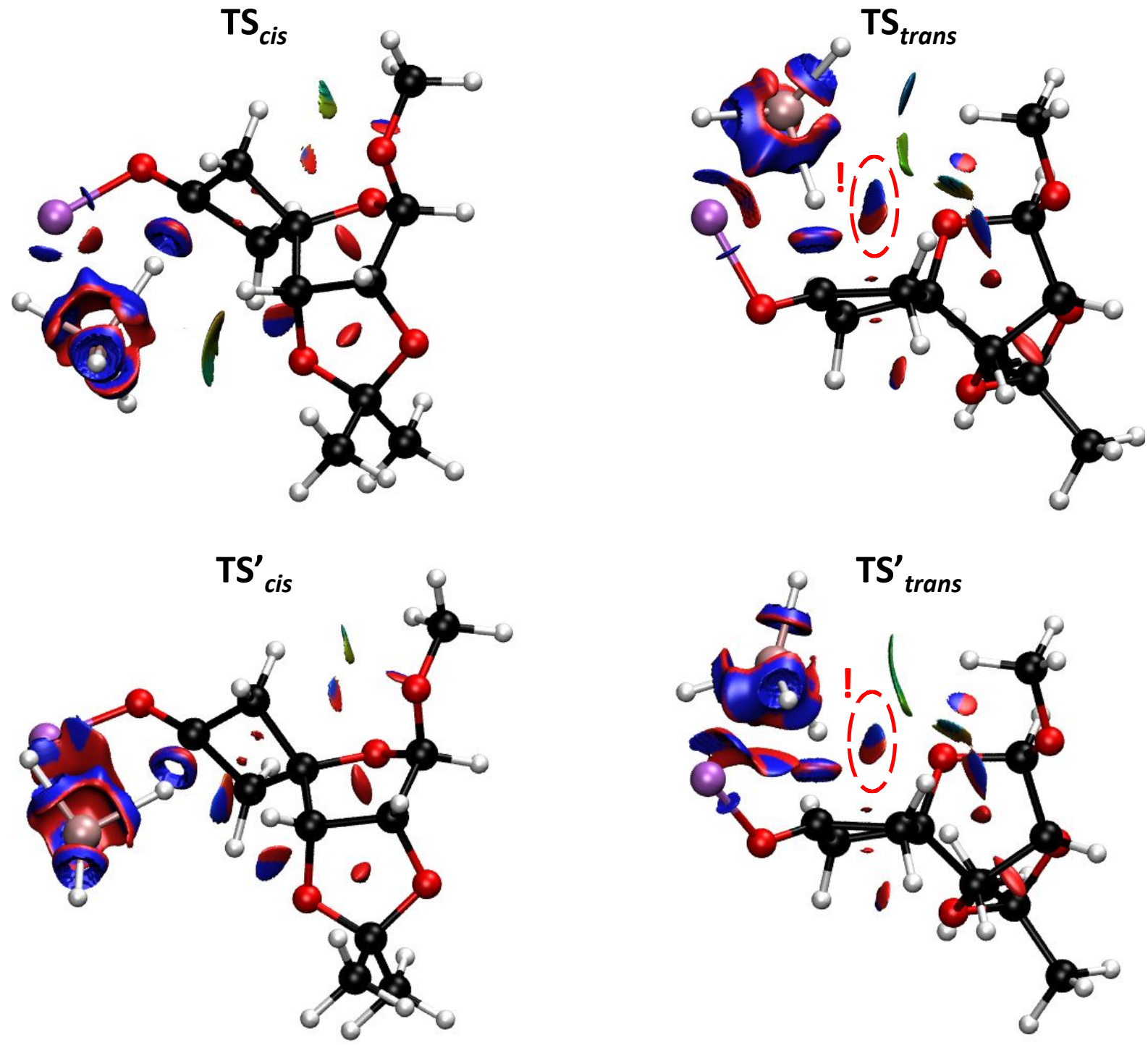

Figure S3. Gradient isosurfaces (isovalue $=0.45$ ) representing the non-covalent interactions occurring in the various transition states describing the reduction of compound 19 with $\mathrm{LiAlH}_{4}$. These isosurfaces are coloured on a RGB scale according to sign $\left(\square_{2}\right) \square \square$ over the range -0.04 a.u. to 0.04 a.u. The red circle marks the repulsive interactions of interest for the observed selectivity.

\section{Reduction of 3- and 3,3-(di)substituted cyclobutanones with $\mathrm{LiAlH}_{4}$}

The reduction of 3-butylcyclobutanone (S1), 3-butoxycyclobutanone (S2), 3tbutylcyclobutanone (S3), 3-butoxy-3-butylcyclobutanone (S4) and 3-butoxy-3tbutylcyclobutanone (S5) (Scheme S1) with the non-sterically hindered $\mathrm{LiAlH}_{4}$ reducing agent was described considering the two previously mentioned bidentate transition $\left(\mathbf{T S}_{\text {cis }}\right.$ and $\mathbf{T S}_{\text {trans }}$ ) states as well as their tridentate (TS' ${ }_{\text {cis }}$ and $\mathbf{T S}{ }_{\text {trans }}$ ) analogues. 


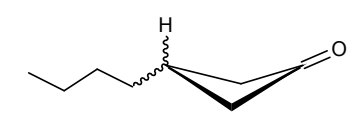

3-butylcyclobutanone (S1)

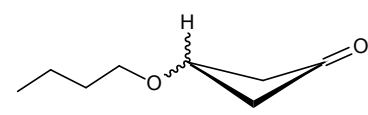

3-butoxycyclobutanone (S2)

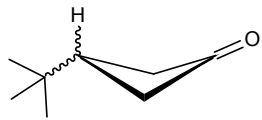

3-tbutylcyclobutanone (S3)

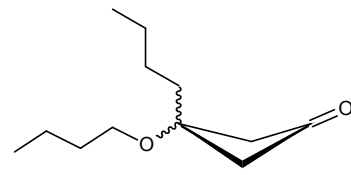

3-butoxy-3-butylcyclobutanone (S4)

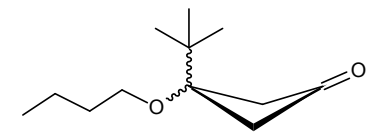

3-butoxy-3-tbutylcyclobutanone (S5)

Scheme S1. Schematic representation of the investigated 3-substituted (S1-S3) and 3,3-disubstituted cyclobutanones (S4-S5).

As indicated in Scheme S2, the cis cyclobutanol isomer of the considered compounds S1 to S5 will be obtained by an anti-facial attack of the hydride relative to the R-substituent for the 3substituted cyclobutanones and the alkoxy-substituent for the 3,3-disubstituted cyclobutanones via transition states $\mathbf{T S}_{\text {cis }}$ and $\mathbf{T S}{ }^{\text {cis. }}$. On the other hand, a syn-facial approach of the reducing agent described by the transition states $\mathbf{T S}_{\text {trans }}$ and $\mathbf{T S}$ ' ${ }_{\text {trans }}$ will give rise to the trans alcohol products. The corresponding relative Gibbs free energies of the different transition states in THF at room and reaction temperature (298.15 and $195.15 \mathrm{~K}$ ) obtained at the B2PLYP-D3/aug-ccpVTZ// $\omega$ B97X-D/cc-pVDZ level of theory are summarized in Table S3.

$\mathrm{TS}_{\text {cis }}$
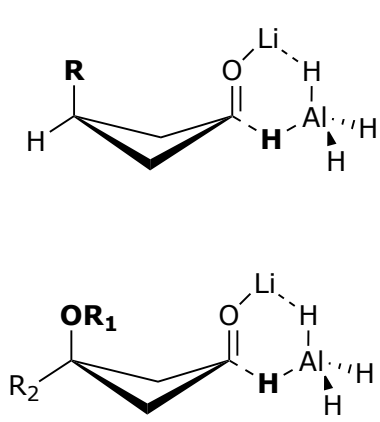

$\mathrm{TS}_{\text {trans }}$
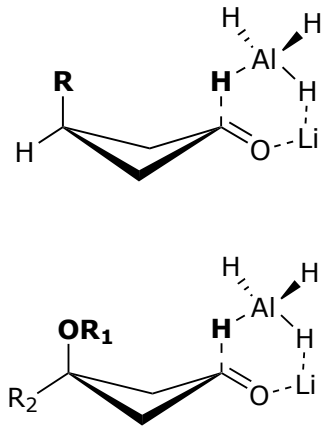

$\mathbf{T S}^{\prime}{ }_{\text {is }}$
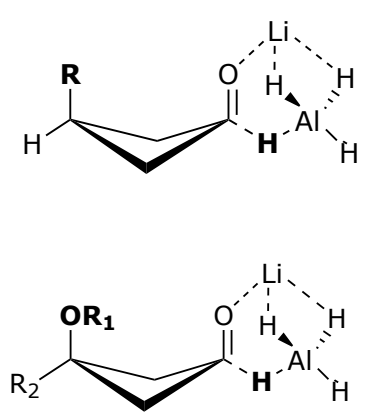

$\mathbf{T S}_{\text {trans }}$
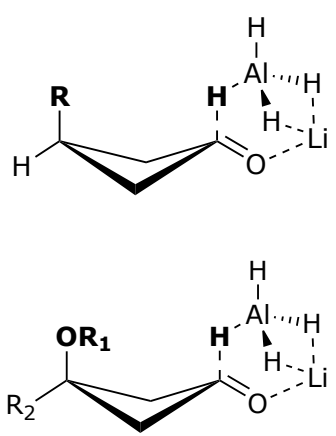

Scheme S2. Schematic representation of the transition states involved in the reduction of 3-substituted (R= butyl, Obutyl and tbutyl) cyclobutanones (top) and 3,3-disubstituted $\left(R_{1}=\right.$ butyl and $R_{2}=$ butyl or tbutyl) cyclobutanones (bottom) with $\mathrm{LiAlH}_{4}$.

From Table S3, it can be observed that for 3-substituted cyclobutanones, regardless of the substituent, the bidentate transition state $\mathbf{T S}_{\text {cis }}$ is characterized by the lowest relative Gibbs free energy. This consequently implies that the reduction of the considered compounds with $\mathrm{LiAlH}_{4}$ will preferentially occur via a transition state in which the hydride approaches the cyclobutanone from the opposite face with respect to the substituent. A preference for such facial approach is also retrieved for the tridentate transition states. Accordingly, these reduction reactions show a pronounced selectivity for the cis alcohol product, as demonstrated by the estimated averaged Boltzmann cis populations ranging between $97 \%$ and $99 \%$. The explicit preference for an antifacial hydride attack towards 3-butyl- and 3-tbutylcyclobutanone appears to be fully attributable to the relief in torsional strain characterized by an increase in puckering angle of $5.5^{\circ}$ and $4.1^{\circ}$, respectively. In addition, the decrease in eclipsed interactions appears to be more distinct in the 


\section{SUPPORTING INFORMATION}

case of a pathway involving the bidentate $\mathbf{T S}_{\text {cis }}$ transition state $\left(\Delta D_{s 1}=-5.7^{\circ}, \Delta D_{s 3}=-6.6^{\circ}\right)$, which probably explains the energetic difference with its tridentate $\mathbf{T S}^{\prime}{ }_{\text {cis }}$ analogue. These structural changes seem not to be recovered via the transition states $\mathbf{T S}_{\text {trans }}$ and $\mathbf{T S}_{\text {trans }}$ since a clear decrease in puckering angle is observed. As such, the four-membered ring structure of 3-tbutylcyclobutanone appears to adopt an almost planar geometry, which can probably be associated to a steric clash between the $t$-butyl substituent and reducing agent upon a syn-facial approach. However, unlike the reduction of the 3-alkyl substituted cyclobutanones, a more puckered butterfly conformation and hence a lowered torsional strain is obtained for all transition states describing the reduction of 3-butoxycyclobutanone, except for the tridentate TS' $_{\text {trans }}$ structure. This increase in puckering angle still appears to be the most pronounced for an antifacial attack of the hydride $\left(\Delta \phi=+3.3^{\circ}\right)$ though it can no longer be considered as the sole driving force behind the pronounced selectivity of this reduction. 
Table S3. Relative Gibbs free energies (in kcal mol-1) together with selected torsional parameters $\left(\phi\right.$ and $D$ in $\left.{ }^{\circ}\right)$ for the different transition states involved in the reduction of compounds S1-S5 with $\mathrm{LiAlH}_{4}$.

\begin{tabular}{|c|c|c|c|c|c|}
\hline 3-butyl (S1) & ref & $\mathbf{T S}_{c i s}$ & $\mathbf{T S}_{\text {trans }}$ & $\mathbf{T S}^{\prime}{ }_{\text {cis }}$ & $\begin{array}{c}\text { TS }_{\text {tran }}^{\prime} \\
s\end{array}$ \\
\hline$\Delta \boldsymbol{G}_{r e e^{[a]}}$ & - & 0.00 & 2.51 & 2.35 & 3.89 \\
\hline$\Delta G_{r e e^{[b]}}$ & - & 0.00 & 2.30 & 1.99 & 3.54 \\
\hline$\phi$ & 13.9 & 19.4 & 11.7 & 19.3 & 11.7 \\
\hline$D$ & 79.7 & 74.0 & 84.2 & 77.6 & 86.7 \\
\hline 3-Obutyl (S2) & ref & $\mathbf{T S}_{\text {cis }}$ & $\mathbf{T S}_{\text {trans }}$ & $\mathbf{T S}^{\prime}{ }_{\text {cis }}$ & $\begin{array}{c}\text { TS }_{\text {tran }}^{\prime} \\
s\end{array}$ \\
\hline$\Delta G_{r e e^{[a]}}$ & - & 0.00 & 4.88 & 2.29 & 3.48 \\
\hline$\Delta G_{r e e^{[b]}}$ & - & 0.00 & 4.15 & 1.98 & 3.17 \\
\hline$\phi$ & 16.6 & 19.8 & 18.7 & 19.7 & 13.6 \\
\hline$D$ & 73.1 & 72.7 & 74.5 & 76.9 & 83.7 \\
\hline 3-tbutyl (S3) & ref & $\mathbf{T S}_{\text {cis }}$ & $\mathbf{T S}_{\text {trans }}$ & $\mathbf{T S}^{\prime}{ }_{\text {cis }}$ & $\begin{array}{c}\text { TS }_{\text {tran }}^{\prime} \\
s\end{array}$ \\
\hline$\Delta G_{r e l}^{[a]}$ & - & 0.00 & 2.20 & 1.90 & 3.57 \\
\hline$\Delta G_{r e e^{[b]}}$ & - & 0.00 & 1.99 & 1.68 & 3.32 \\
\hline$\phi$ & 16.2 & 20.3 & 0.2 & 20.0 & 1.5 \\
\hline$D$ & 80.9 & 74.3 & 96.7 & 77.7 & 96.8 \\
\hline 3-OButyl/Butyl (S4) & ref & $\mathbf{T S}_{c i s}$ & $\mathrm{TS}_{\text {trans }}$ & $\mathbf{T S}^{\prime}{ }_{\text {cis }}$ & $\begin{array}{c}\text { TS }_{\text {'tran }} \\
s\end{array}$ \\
\hline$\Delta G_{r e l}^{[a]}$ & - & 0.00 & 2.72 & 1.54 & 2.42 \\
\hline$\Delta G_{r e l^{[b]}}$ & - & 0.00 & 2.22 & 1.34 & 2.15 \\
\hline$\phi$ & 16.5 & 12.3 & 15.7 & 12.8 & 13.9 \\
\hline$D$ & 83.7 & 82.3 & 80.5 & 84.4 & 85.0 \\
\hline 3-OButyl/tbutyl (S5) & ref & $\mathbf{T S}_{c i s}$ & $\mathbf{T S}_{\text {trans }}$ & $\mathbf{T S}_{\text {cis }}^{\prime}$ & $\begin{array}{c}\text { TS }_{\text {tran }}^{\prime} \\
s\end{array}$ \\
\hline$\Delta G_{\text {rel }}{ }^{[a]}$ & - & 0.00 & 3.12 & 2.54 & 0.88 \\
\hline$\Delta G_{r e l^{[b]}}$ & - & 0.00 & 2.81 & 2.42 & 0.92 \\
\hline$\phi$ & 19.3 & 12.3 & 12.8 & 0.9 & 0.4 \\
\hline$D$ & 84.0 & 101.1 & 87.8 & 97.9 & 99.7 \\
\hline
\end{tabular}

[a] Energetic data computed at $298.15 \mathrm{~K}$ with the [SMD(THF)]/B2PLYP-D3/aug-cc-pVTZ// $\omega B$ B7X-D/cc-pVDZ level of theory. [b] Energetic data computed at $195.15 \mathrm{~K}$ with the $[\mathrm{SMD}(\mathrm{THF})] / \mathrm{B} 2 \mathrm{PLYP}-\mathrm{D} 3 /$ aug-cc-pVTZ// $\omega$ B97X-D/cc-pVDZ level of theory.

The analysis of the gradient isosurfaces representing the noncovalent interactions (Figure S4) occurring during the reduction of 3-butoxycyclobutanone revealed a pronounced repulsive interaction (red surface) between the $\mathrm{AlH}_{4}^{-}$moiety of the reducing agent and the oxygen atom emanating from the butoxy-substituent for the syn-facial attack via $\mathbf{T S}_{\text {trans }}$. This isosurface is to a lesser extent also present for the tridentate $\mathbf{T S}$ 'trans structure, altough a more flattened conformation with increased eclipsed interactions is obtained for the latter. On the other hand, the anti-facial hydride approach via both transition states $\mathbf{T S}_{\text {cis }}$ and $\mathbf{T} \mathbf{S}{ }_{c i s}$ does not appear to be 


\section{SUPPORTING INFORMATION}

influenced by any repulsive interaction originating from the substituent and also entails a lowering of the torsional strain. Therefore, it can be concluded that the strong selectivity of the reduction of 3-butoxycyclobutanone towards its cis cyclobutanol product is influenced by a subtle synergy between both torsional strain and electrostatic repulsive interactions originating from the substituent. The pronounced preference for a pathway in which the hydride approaches 3-tbutylcyclobutanone via the side opposite to the $t$-butyl substituent is driven by minimizing the repulsive interactions in addition to the increase in ring puckering. In this respect, Figure S6 clearly demonstrates that a syn-facial attack via the transition states $\mathbf{T S}_{\text {trans }}$ and $\mathbf{T S}_{\text {'trans }}$ exhibits a very distinct steric interference between the substituent and the reducing agent, while in the case of an anti-facial approach this is no longer the case. A similar, but less pronounced, effect is also noticeable for 3-butylcyclobutanone (Figure S8). Consequently, it can be assumed that the stereochemical outcome of 3-alkyl substituted cyclobutanone reductions is mainly driven by the reduction of torsional strain as described in the Felkin-Ahn model and to a lesser extent by steric hindrance.

In order to determine which of the above-mentioned effects has the greatest influence on the selectivity, the reduction of both 3-butoxy-3-butylcyclobutanone (S4) and 3-butoxy3tbutylcyclobutanone (S5) with $\mathrm{LiAlH}_{4}$ was studied. As expected, $\mathbf{T S}_{\text {cis }}$ corresponds to the lowest energy structure favouring as such the anti-facial approach of the hydride with respect to the alkoxy-moiety. Whereas the reduction of 3-butoxy-3-butylcyclobutanone at room temperature still gives rise to a very high amount of cis isomer (96\%), it turns out that the selectivity of the reduction of 3-butoxy-3-tbutylcyclobutanone is significantly less pronounced $(82 \%)$ suggesting a possible interplay of different effects. Nevertheless, unlike the previous 3substituted cyclobutanones, the torsional strain of the four-membered ring structure does not appear to play any role in the selectivity of these reductions, since the puckering angle $\phi$ decreases with respect to the initial reagents regardless of an anti- or syn-facial hydride attack. Furthermore, Table S3 reveals that for both the reduction of compounds S4 $\left(\Delta \phi=-0.8^{\circ}\right)$ and S5 $\left(\Delta \phi=-6.5^{\circ}\right)$ the least pronounced flattening of the butterfly conformation will occur when the reduction follows a pathway involving $\mathbf{T S}_{\text {trans }}$, while this transition structure is characterized by the highest relative Gibbs free energy of $2.22 \mathrm{kcal} \mathrm{mol}^{-1}$ and $2.81 \mathrm{kcal} \mathrm{mol}^{-1}$, respectively.

In addition, the isosurfaces (Figure S10 and S11) for the bidentate and tridentate $\mathbf{T S}_{\text {cis }}$ transition states always exhibit repulsive interactions attributable to the bulk of the alkyl-substituent, with the impact of the tert-butyl group being more significant. Furthermore, a syn-facial hydride attack of the hydride seems to be always confronted with the repulsive electrostatic interactions induced by the proximity of the electronegative oxygen atom of the alkoxy-substituent and the reducing agent. However, from the two-dimensional $\mathrm{NCl}$ plot (Figure S12) representing the interactions occurring in the lowest energy transition state $\mathbf{T S}_{\text {cis }}$ for the reduction of 3-butoxy-3butylcyclobutanone (green surface) and 3-butoxy-3-tbutylcyclobutanone (red surface), it can be 


\section{SUPPORTING INFORMATION}

observed that the reduction of the latter compound is accompanied by repulsive interactions that are spread over a wider range as compared to the reduction of 3-butoxy-3butylcyclobutanone. Consequently, it can be hypothesized that one specific type of repulsive interaction, i.e. the electrostatic repulsion originating from the alkoxy-substituent, mainly controls the stereochemical outcome of the reduction of 3-butoxy-3-butylcyclobutanone, whereas the reduction of 3-butoxy-3-tbutylcyclobutanone requires a trade-off between a minimal steric hindrance, on the one hand, and minimal electrostatic repulsion, on the other.
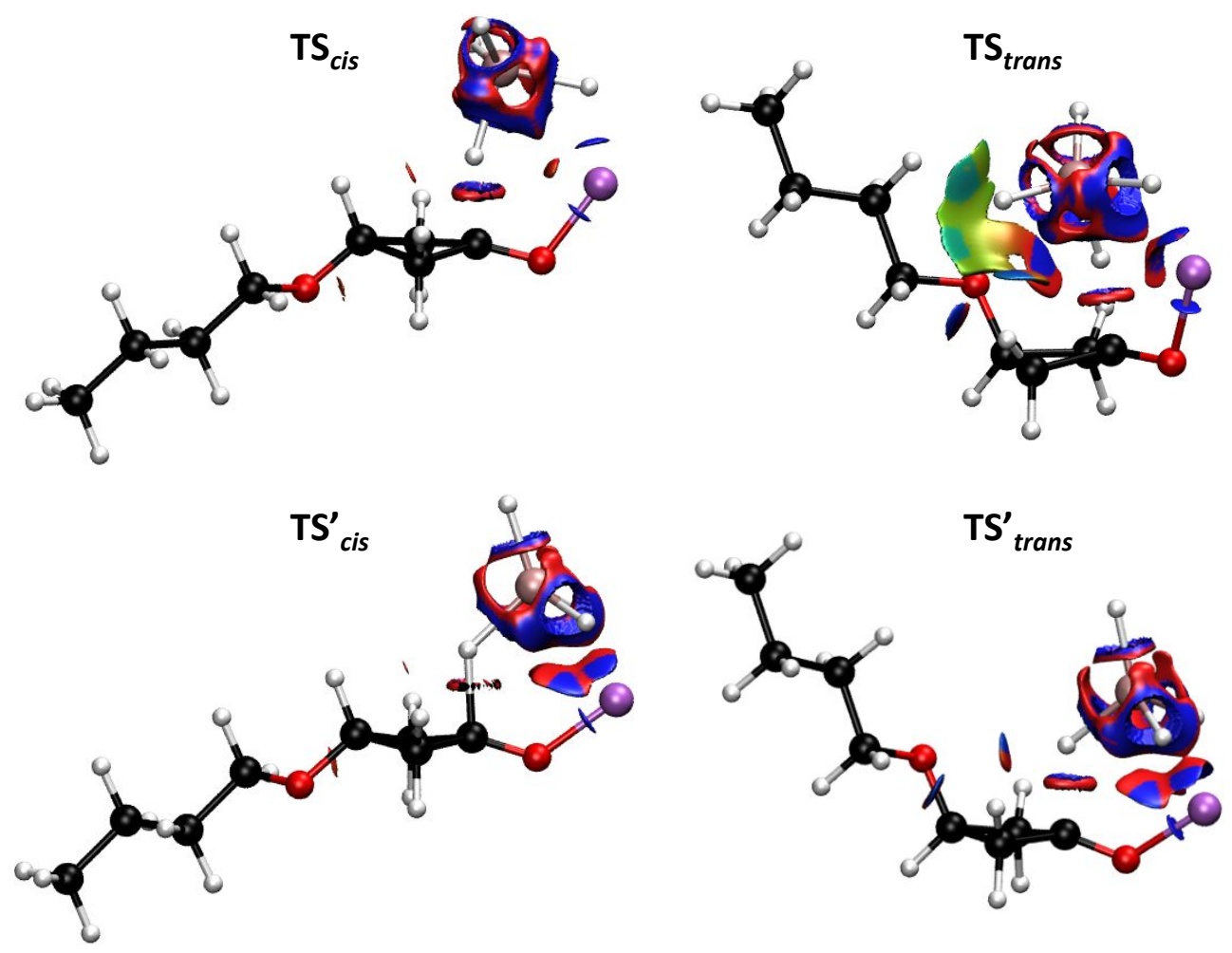

Figure S4. Gradient isosurfaces (isovalue $=0.45$ ) representing the non-covalent interactions occurring in the various transition states describing the reduction of 3-butoxycyclobutanone with $\mathrm{LiAlH}_{4}$. These isosurfaces are coloured on a RGB scale according to $\operatorname{sign}\left(\lambda_{2}\right) \rho$ over the range 0.04 a.u. to 0.04 a.u. 


\section{SUPPORTING INFORMATION}

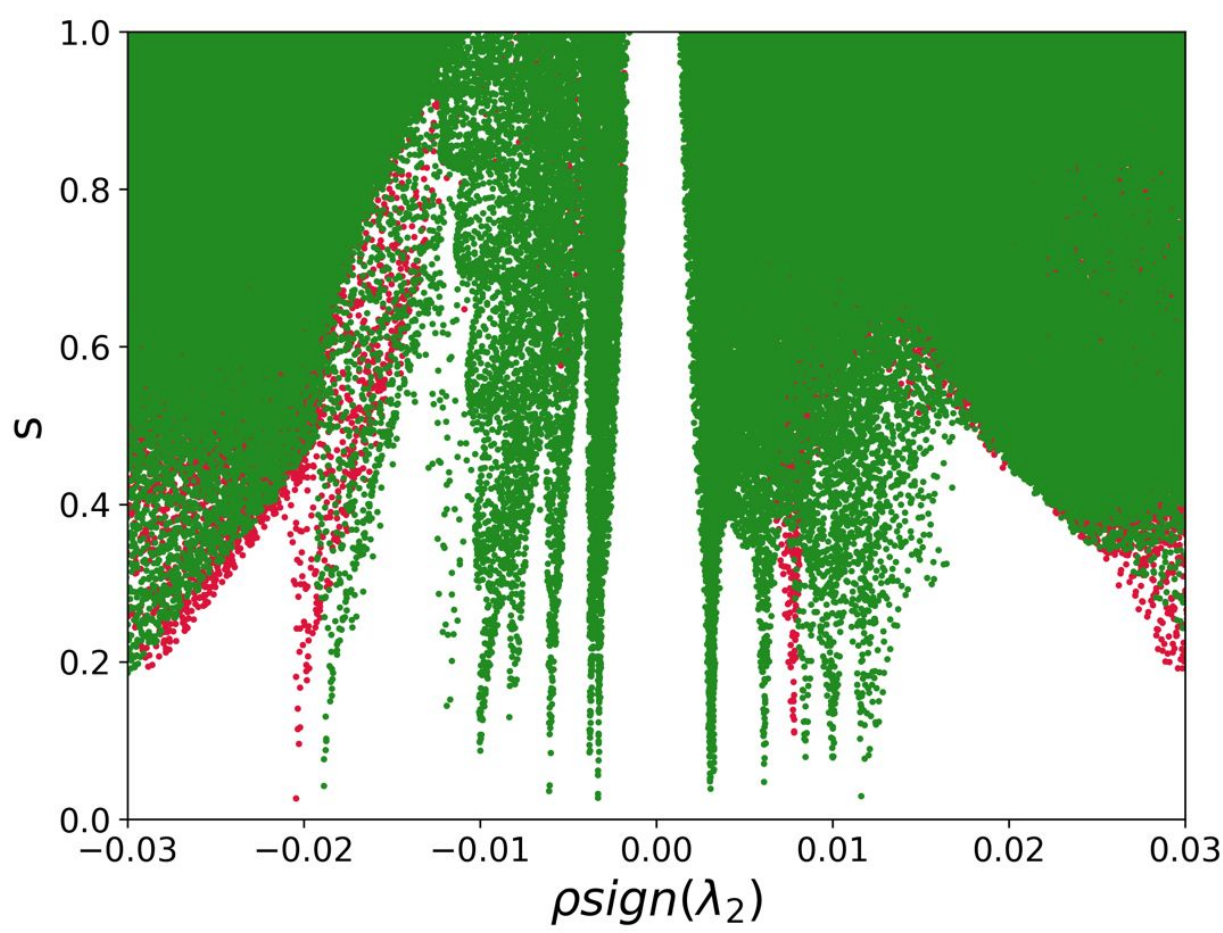

Figure S5. Bidimensional plot of the reduced density gradient $v s$ the electron density multiplied by the sign of the second Hessian eigenvalue comparing the strength of the attractive and repulsive interactions in the bidentate transition states $\mathbf{T S}_{\text {cis }}$ (red) and $\mathbf{T} \mathbf{S}_{\text {trans }}$ ( $(\mathrm{green})$ for the reduction of 3-butoxycyclobutanone with $\mathrm{LiAlH}_{4}$.
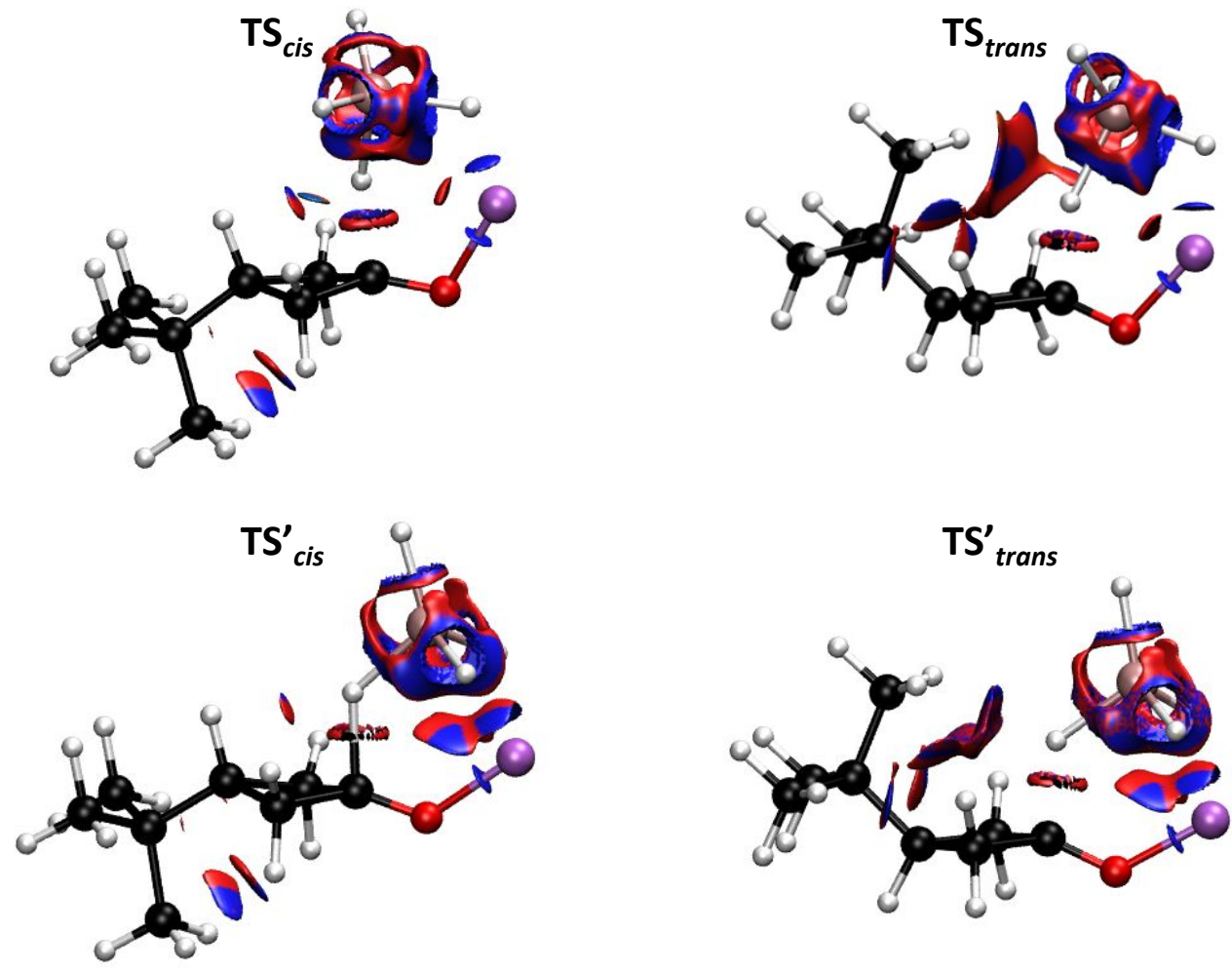

Figure S6. Gradient isosurfaces (isovalue $=0.45$ ) representing the non-covalent interactions occurring in the various transition states describing the reduction of 3-tbutylcyclobutanone with $\mathrm{LiAlH}_{4}$. These isosurfaces are coloured on a RGB scale according to sign $\left(\lambda_{2}\right) \rho$ over the range -0.04 a.u. to 0.04 a.u. 


\section{SUPPORTING INFORMATION}

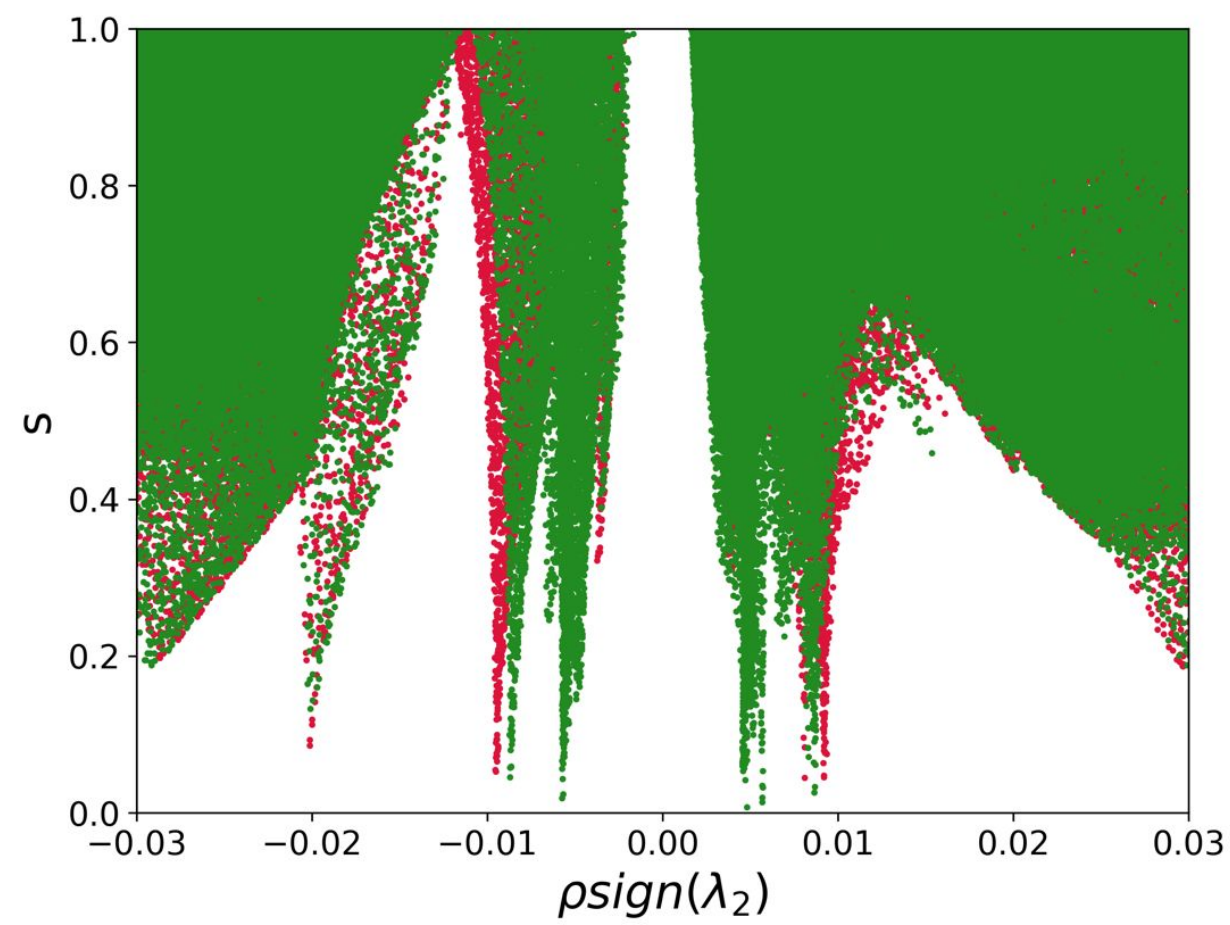

Figure S7. Bidimensional plot of the reduced density gradient $v s$ the electron density multiplied by the sign of the second Hessian eigenvalue comparing the strength of the attractive and repulsive interactions in the bidentate transition states $\mathbf{T S}_{\text {cis }}$ (red) and $\mathbf{T \mathbf { S } _ { \text { trans } }}$ (green) for the reduction of 3-tbutylcyclobutanone with $\mathrm{LiAlH}_{4}$.
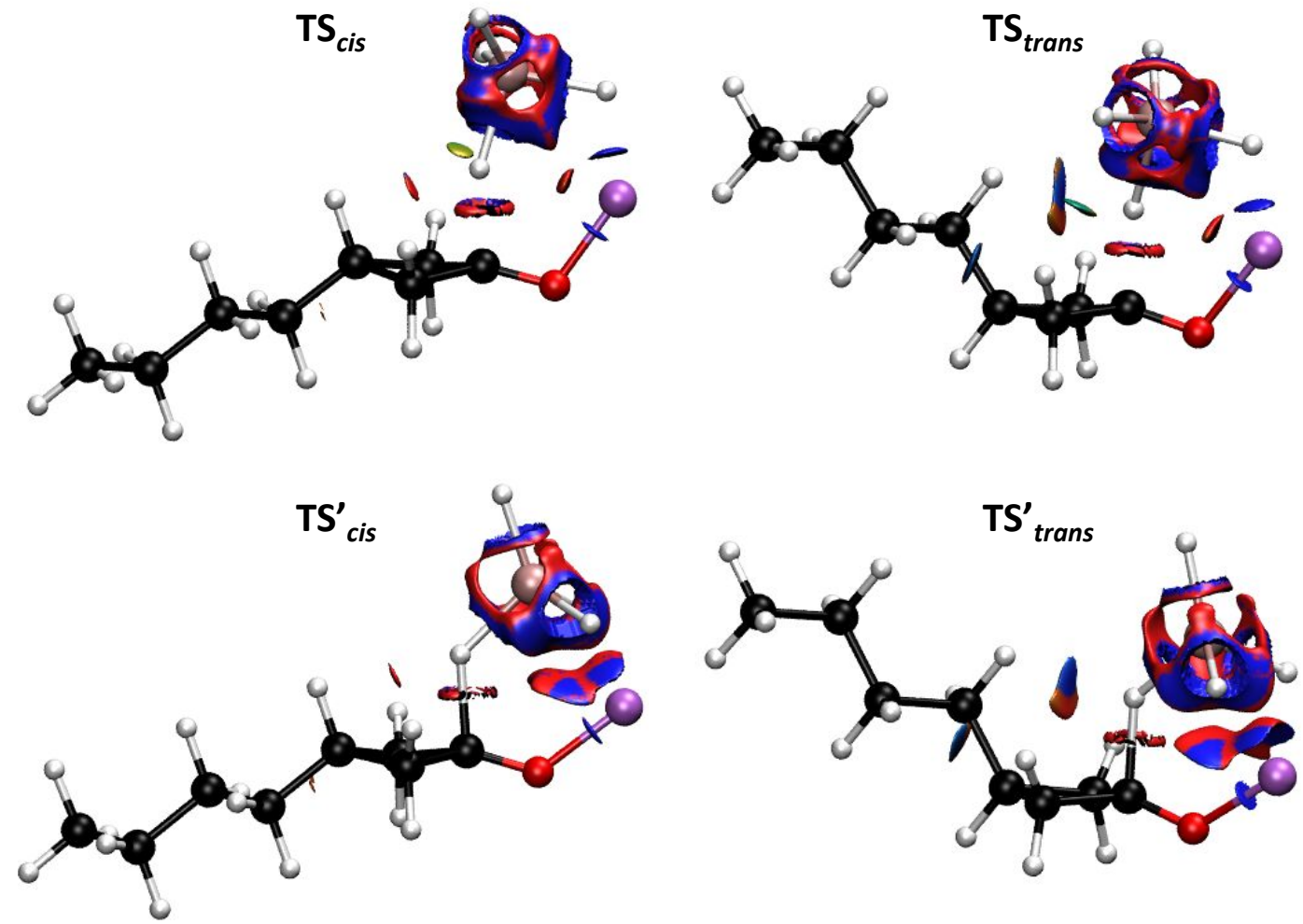

Figure S8. Gradient isosurfaces (isovalue $=0.45$ ) representing the non-covalent interactions occurring in the various transition states describing the reduction of 3-butylcyclobutanone with $\mathrm{LiAlH}_{4}$. These isosurfaces are coloured on a RGB scale according to sign $\left(\lambda_{2}\right) \rho$ over the range -0.04 a.u. to 0.04 a.u. 


\section{SUPPORTING INFORMATION}

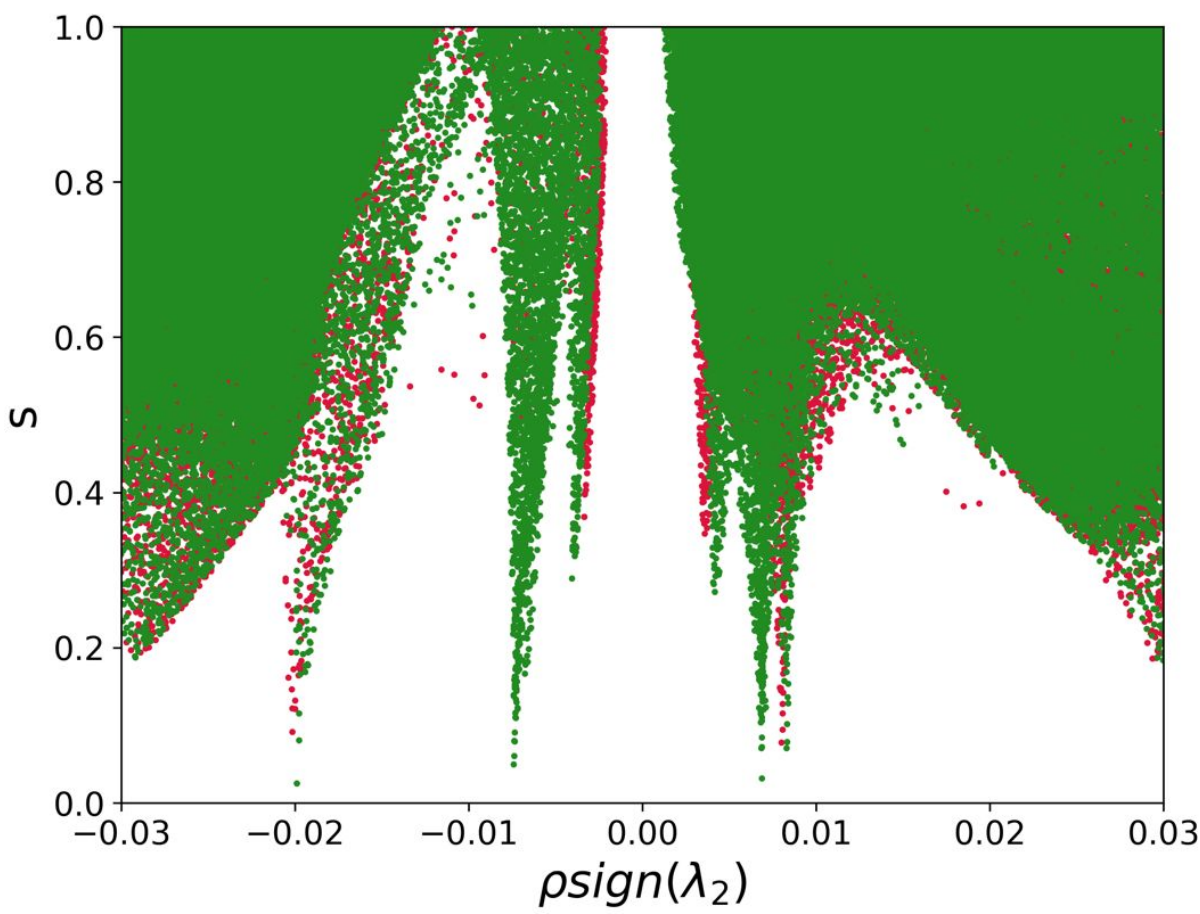

Figure S9. Bidimensional plot of the reduced density gradient $v$ s the electron density multiplied by the sign of the second Hessian eigenvalue comparing the strength of the attractive and repulsive interactions in the bidentate transition states $\mathbf{T S}_{\text {cis }}$ (red) and $\mathbf{T} \mathbf{S}_{\text {trans }}$ ( $\mathrm{green}$ ) for the reduction of 3-butylcyclobutanone with $\mathrm{LiAlH}_{4}$.
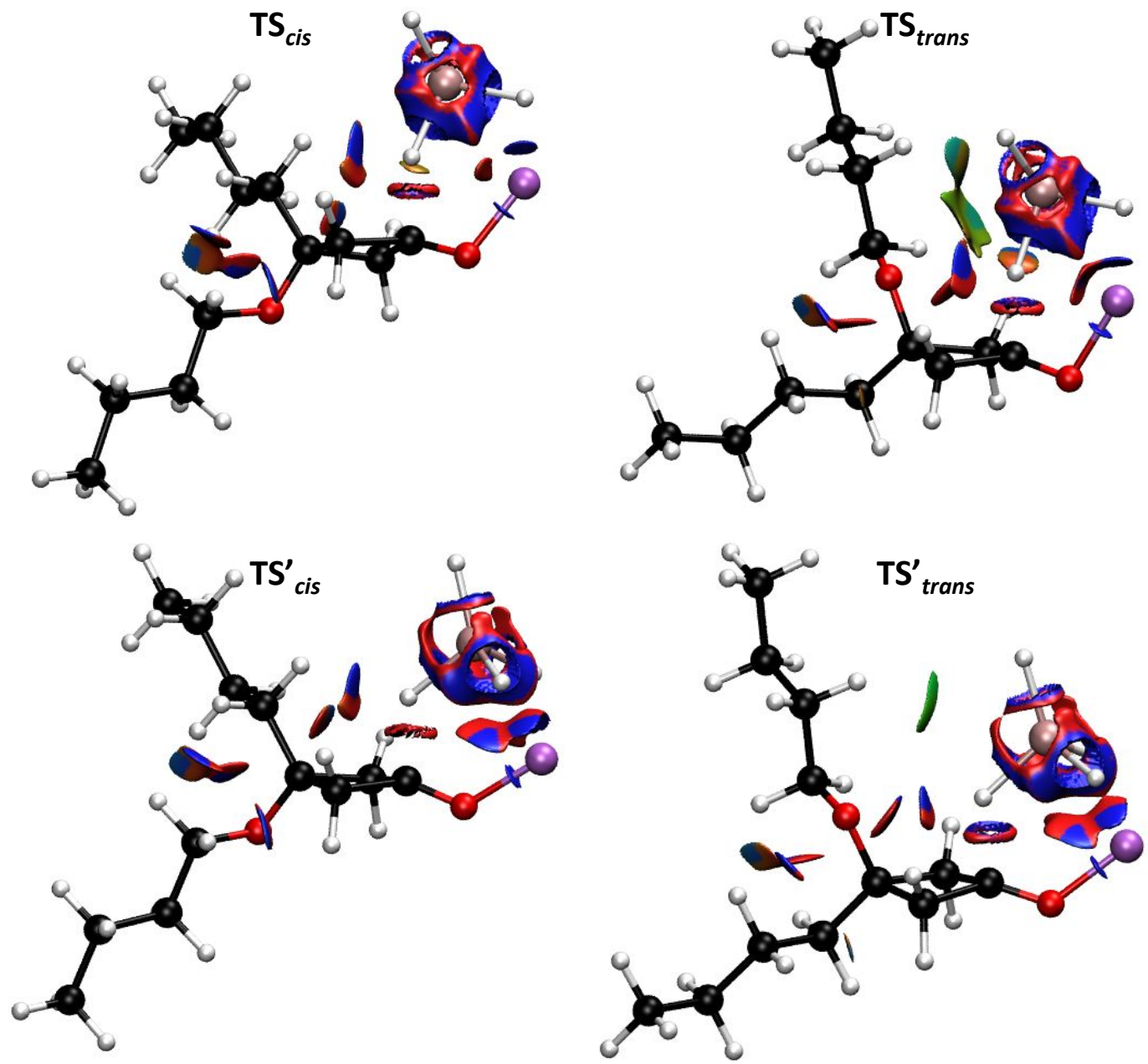


\section{SUPPORTING INFORMATION}

Figure S10. Gradient isosurfaces (isovalue $=0.45$ ) representing the non-covalent interactions occurring in the various transition states describing the reduction of 3-butoxy-3-butylcyclobutanone with $\mathrm{LiAlH}_{4}$. These isosurfaces are coloured on a $\mathrm{RGB}$ scale according to $\operatorname{sign}\left(\lambda_{2}\right) \rho$ over the range -0.04 a.u. to 0.04 a.u.
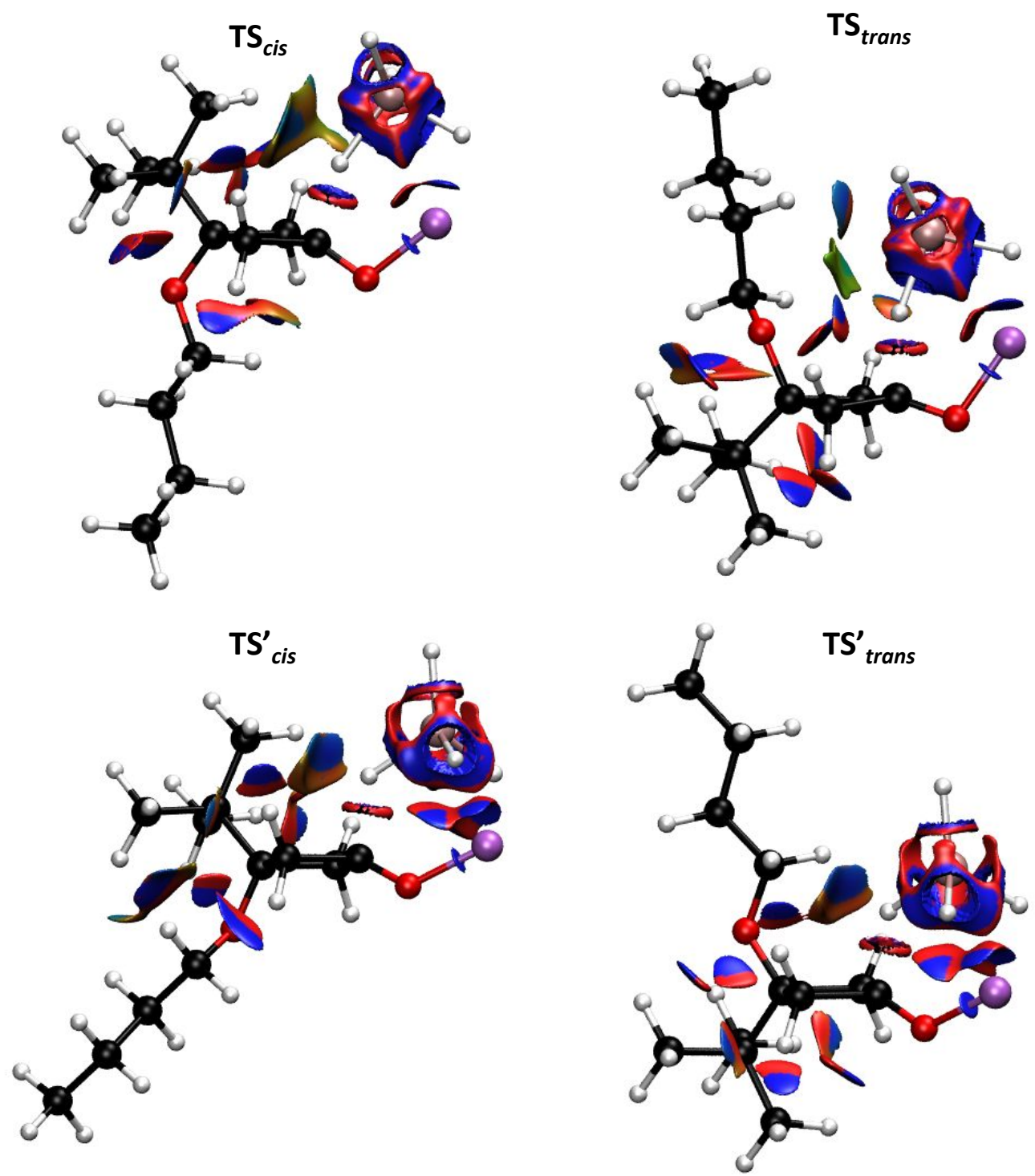

Figure S11. Gradient isosurfaces (isovalue $=0.45$ ) representing the non-covalent interactions occurring in the various transition states describing the reduction of 3-butoxy-3-tbutylcyclobutanone with $\mathrm{LiAlH}_{4}$. These isosurfaces are coloured on a RGB scale according to $\operatorname{sign}\left(\lambda_{2}\right) \rho$ over the range -0.04 a.u. to 0.04 a.u. 


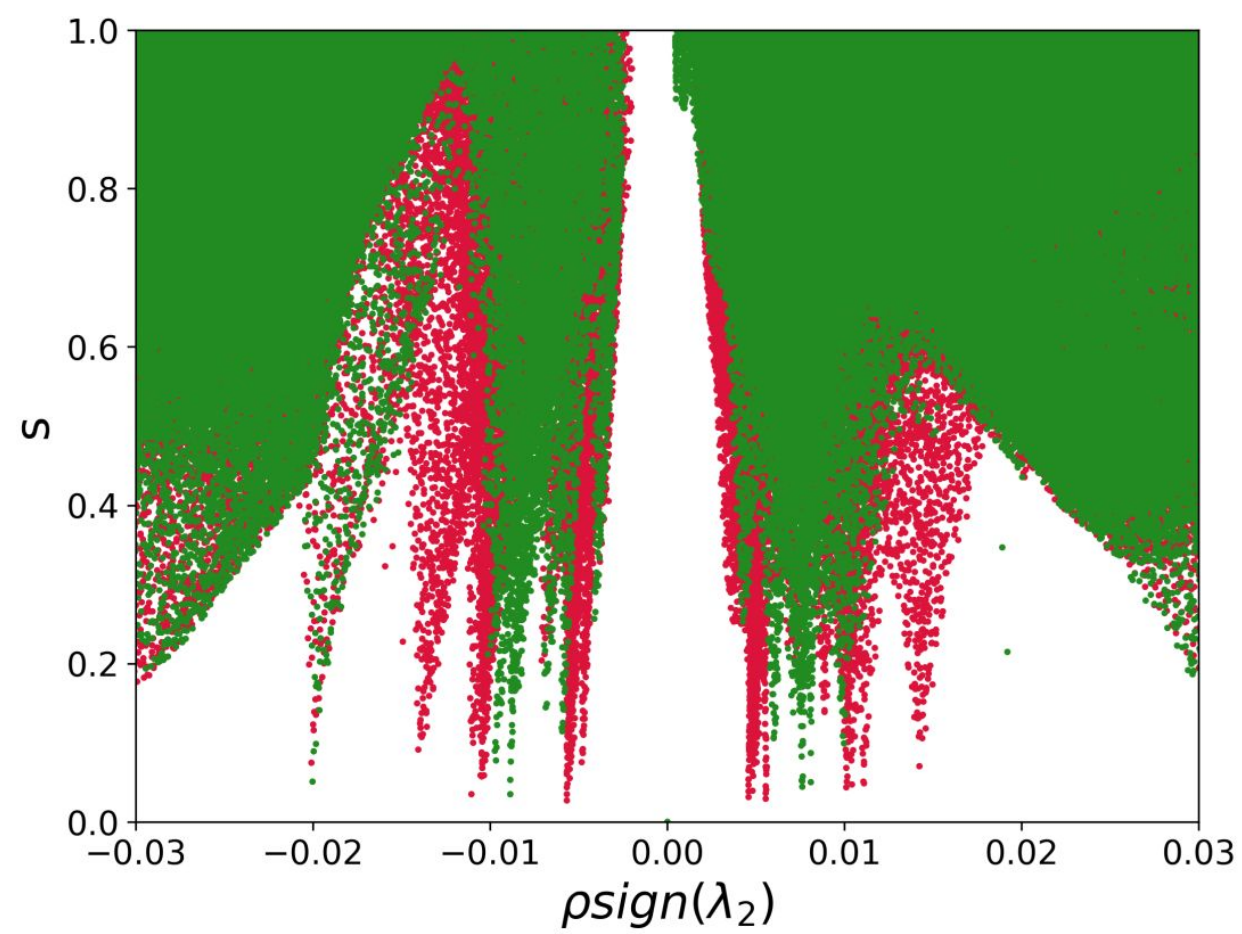

Figure S12. Bidimensional plot of the reduced density gradient $v s$ the electron density multiplied by the sign of the second Hessian eigenvalue comparing the strength of the attractive and repulsive interactions in the lowest energy transition state $\mathbf{T S}_{\text {cis }}$ for the reduction of 3-butoxy-3butylcyclobutanone (green) and 3-butoxy-3-tbutylcyclobutanone (red) with $\mathrm{LiAlH}_{4}$.

\section{$\underline{\mathrm{XYZ} \text {-coordinates for the various transition states: }}$}

\begin{tabular}{llll}
\multicolumn{1}{l}{ TS $_{\text {trans_ }}$ Bbutyl } \\
$\mathrm{C}$ & -0.79158300 & -0.99909800 & -1.05831700 \\
$\mathrm{H}$ & -0.40078600 & -0.10864200 & -1.56848800 \\
$\mathrm{H}$ & -0.99369600 & -1.78184300 & -1.80593500 \\
$\mathrm{C}$ & -1.35464800 & -1.52517600 & 1.00653600 \\
$\mathrm{H}$ & -1.79508100 & -2.52786900 & 1.12102800 \\
$\mathrm{H}$ & -1.36114500 & -1.01634000 & 1.97905900 \\
$\mathrm{C}$ & -2.03232300 & -0.81911300 & -0.17606000 \\
$\mathrm{O}$ & -3.24341700 & -0.81834100 & -0.50873300 \\
$\mathrm{Li}$ & -3.90551300 & 0.85687700 & -0.45053600 \\
$\mathrm{H}$ & -1.71547500 & 0.70129900 & 0.33587900 \\
$\mathrm{Al}$ & -1.64862200 & 2.40597700 & 0.17846700 \\
$\mathrm{H}$ & -1.40948900 & 3.04732300 & 1.62313600 \\
$\mathrm{H}$ & -0.65330300 & 2.67773800 & -1.04738800 \\
$\mathrm{H}$ & -3.25075200 & 2.56259500 & -0.30235000 \\
$\mathrm{C}$ & -0.02454600 & -1.51373100 & 0.19764200 \\
$\mathrm{H}$ & 0.39651200 & -2.52013800 & 0.05371200 \\
$\mathrm{C}$ & 1.05836500 & -0.58584200 & 0.73798800
\end{tabular}




\section{SUPPORTING INFORMATION}

$\begin{array}{lrrr}\mathrm{C} & 2.28269800 & -0.49302800 & -0.16781000 \\ \mathrm{H} & 1.36524300 & -0.94479800 & 1.73652300 \\ \mathrm{H} & 0.63817600 & 0.42322500 & 0.89091200 \\ \mathrm{C} & 3.34910200 & 0.46577500 & 0.35765500 \\ \mathrm{H} & 2.72225200 & -1.49957000 & -0.29493000 \\ \mathrm{H} & 1.97069700 & -0.16649600 & -1.17633500 \\ \mathrm{C} & 4.57151200 & 0.55499800 & -0.55064800 \\ \mathrm{H} & 2.90282900 & 1.46817900 & 0.47856600 \\ \mathrm{H} & 3.65959500 & 0.14557400 & 1.36788600 \\ \mathrm{H} & 5.32181700 & 1.25298200 & -0.15001900 \\ \mathrm{H} & 5.05520500 & -0.42881600 & -0.66440300 \\ \mathrm{H} & 4.29189200 & 0.90528000 & -1.55737100\end{array}$

1 imaginary frequency: $-396.9468 \mathrm{~cm}^{-1}$

TC[G] $(\omega B 97 X-D / c c-p V D Z$ at $298.15 \mathrm{~K})=0.192767 \mathrm{au}$

TC[G] $(\omega B 97 X-D / c c-p V D Z$ at $195.15 \mathrm{~K})=0.211222$ au

$E$ (B2PLYP-D3/ aug-cc-pVTZ in gas phase $)=-639.963931$ au

E (B2PLYP-D3/ aug-cc-pVTZ in THF) $=-639.982573$ au

\section{TS cis_3butyl $_{3}$}

$\begin{array}{lrrr}\text { C } & -0.38645200 & -0.59120400 & -0.92447800 \\ \text { H } & -0.46538300 & 0.06195000 & -1.80161300 \\ \text { H } & 0.13851000 & -1.52006500 & -1.20927200 \\ \mathrm{C} & -0.98948900 & -0.75646500 & 1.17286100 \\ \mathrm{H} & -0.65853500 & -1.73618300 & 1.55861300 \\ \mathrm{H} & -1.54700900 & -0.22672000 & 1.95505200 \\ \mathrm{C} & -1.66494500 & -0.99012800 & -0.18087600 \\ \mathrm{O} & -2.56854000 & -1.79821400 & -0.50244200 \\ \mathrm{Li} & -4.14928900 & -0.95809100 & -0.71741200 \\ \mathrm{H} & -2.33698100 & 0.51165200 & -0.29605500 \\ \mathrm{Al} & -3.53086500 & 1.69567900 & 0.01953100 \\ \mathrm{H} & -3.47879000 & 1.95987300 & 1.59877900 \\ \mathrm{H} & -3.38619800 & 2.87822700 & -1.04680800 \\ \mathrm{H} & -4.82691600 & 0.69699300 & -0.36699900 \\ \mathrm{C} & 0.14947000 & -0.01820500 & 0.41824100 \\ \mathrm{H} & -0.01390400 & 1.06975600 & 0.45634400 \\ \mathrm{C} & 1.58336500 & -0.34127800 & 0.80198200 \\ \mathrm{C} & 2.61254300 & 0.32710000 & -0.10656600 \\ \mathrm{H} & 1.72799400 & -1.43748900 & 0.77776200 \\ \mathrm{H} & 1.75693000 & -0.03120300 & 1.84754200\end{array}$




\section{SUPPORTING INFORMATION}

$\begin{array}{lrrr}\mathrm{C} & 4.05681300 & -0.00132800 & 0.26579100 \\ \mathrm{H} & 2.42862100 & 0.02340800 & -1.15352900 \\ \mathrm{H} & 2.46663400 & 1.42221600 & -0.07849900 \\ \mathrm{C} & 5.07720800 & 0.67055200 & -0.64797600 \\ \mathrm{H} & 4.23903900 & 0.30204100 & 1.31174000 \\ \mathrm{H} & 4.19818800 & -1.09621300 & 0.23779100 \\ \mathrm{H} & 6.10782300 & 0.41702100 & -0.35787800 \\ \mathrm{H} & 4.93729200 & 0.35905100 & -1.69577800 \\ \mathrm{H} & 4.97905100 & 1.76757500 & -0.61318800\end{array}$

1 imaginary frequency: $-387.0274 \mathrm{~cm}^{-1}$

TC[G] ( $\omega B$ B97X-D/cc-pVDZ at $298.15 \mathrm{~K})=0.191610 \mathrm{au}$ TC[G] ( $\omega B$ B97X-D/cc-pVDZ at $195.15 \mathrm{~K})=0.210420 \mathrm{au}$ $\mathrm{E}$ (B2PLYP-D3/ aug-cc-pVTZ in gas phase) $=-639.966215$ au E (B2PLYP-D3/ aug-cc-pVTZ in THF) $=-639.985427$ au

\section{TS' trans_3butyl}

$\begin{array}{llll}\mathrm{C} & -0.57034900 & -0.97985000 & -1.04478000 \\ \mathrm{H} & -0.21837600 & -0.09469100 & -1.59042400 \\ \mathrm{H} & -0.73109800 & -1.79962900 & -1.76183600 \\ \mathrm{C} & -1.11358200 & -1.45972500 & 1.03813300 \\ \mathrm{H} & -1.50166100 & -2.47898900 & 1.18800200 \\ \mathrm{H} & -1.14677100 & -0.91975700 & 1.99312900 \\ \mathrm{C} & -1.82543400 & -0.82783100 & -0.16948100 \\ \mathrm{O} & -3.02577100 & -0.94485200 & -0.50910200 \\ \mathrm{Li} & -4.21842300 & 0.40355400 & -0.67464000 \\ \mathrm{H} & -1.51599000 & 0.69409300 & 0.27068400 \\ \mathrm{Al} & -2.46183400 & 2.08686200 & 0.18940800 \\ \mathrm{H} & -1.75692100 & 3.43892200 & 0.64773800 \\ \mathrm{H} & -3.00919900 & 1.93136500 & -1.36287400 \\ \mathrm{H} & -3.79650000 & 1.53523200 & 0.99345200 \\ \mathrm{C} & 0.21604100 & -1.41163000 & 0.23015900 \\ \mathrm{H} & 0.68644200 & -2.40084900 & 0.12291800 \\ \mathrm{C} & 1.25261500 & -0.41452800 & 0.73769000 \\ \mathrm{C} & 2.48895500 & -0.32447100 & -0.15262700 \\ \mathrm{H} & 1.55678500 & -0.70883600 & 1.75810500 \\ \mathrm{H} & 0.78943300 & 0.58315900 & 0.82942300 \\ \mathrm{C} & 3.51637100 & 0.69300300 & 0.33937500 \\ \mathrm{H} & 2.96328500 & -1.32074700 & -0.22217600 \\ \mathrm{H} & 2.18397600 & -0.06168600 & -1.18202500\end{array}$




\section{SUPPORTING INFORMATION}

$\begin{array}{llrr}\mathrm{C} & 4.75015200 & 0.77713800 & -0.55406500 \\ \mathrm{H} & 3.03809300 & 1.68585100 & 0.40599400 \\ \mathrm{H} & 3.82061200 & 0.43254800 & 1.36845900 \\ \mathrm{H} & 5.47196600 & 1.51729000 & -0.17740400 \\ \mathrm{H} & 5.26583000 & -0.19517600 & -0.61191200 \\ \mathrm{H} & 4.47676000 & 1.06821200 & -1.58124300\end{array}$

1 imaginary frequency: $-501.9764 \mathrm{~cm}^{-1}$

TC[G] ( $\omega B$ B7X-D/cc-pVDZ at $298.15 \mathrm{~K})=0.193791 \mathrm{au}$ TC[G] ( $\omega B$ 97X-D/cc-pVDZ at $195.15 \mathrm{~K})=0.212045 \mathrm{au}$ $E$ (B2PLYP-D3/ aug-cc-pVTZ in gas phase) $=-639.964553$ au E (B2PLYP-D3/ aug-cc-pVTZ in THF) $=-639.981409$ au

\begin{tabular}{lrrr}
\multicolumn{4}{c}{ TS $^{\prime}{ }_{\text {is_ }}$ 3butyl } \\
$\mathrm{C}$ & -0.88951400 & -1.00131400 & -1.06271400 \\
$\mathrm{H}$ & -1.37127200 & -0.77629600 & -2.02176200 \\
$\mathrm{H}$ & -0.58838200 & -2.06283200 & -1.04899500 \\
$\mathrm{C}$ & -0.39432800 & -0.11210300 & 0.87593000 \\
$\mathrm{H}$ & 0.06620400 & -0.89012200 & 1.50948000 \\
$\mathrm{H}$ & -0.48505600 & 0.81503800 & 1.45433600 \\
$\mathrm{C}$ & -1.65419400 & -0.71413200 & 0.23746700 \\
$\mathrm{O}$ & -2.57524200 & -1.37115600 & 0.77455000 \\
$\mathrm{Li}$ & -4.29607500 & -0.84996800 & 0.94518800 \\
$\mathrm{H}$ & -2.20099600 & 0.71680400 & -0.27720900 \\
$\mathrm{Al}$ & -3.74573500 & 1.39147400 & -0.21769500 \\
$\mathrm{H}$ & -3.90257800 & 2.84398900 & -0.84950700 \\
$\mathrm{H}$ & -4.60087500 & 0.12483600 & -0.84740100 \\
$\mathrm{H}$ & -4.06719900 & 1.15245200 & 1.38585400 \\
$\mathrm{C}$ & 0.24397300 & -0.07636600 & -0.54168700 \\
$\mathrm{H}$ & 0.14496200 & 0.93029800 & -0.97630900 \\
$\mathrm{C}$ & 1.67709800 & -0.56100900 & -0.68290000 \\
$\mathrm{C}$ & 2.69103900 & 0.35469300 & -0.00114600 \\
$\mathrm{H}$ & 1.92551500 & -0.65334800 & -1.75515400 \\
$\mathrm{H}$ & 1.75907100 & -1.57937200 & -0.25947700 \\
$\mathrm{C}$ & 4.13282900 & -0.13286300 & -0.12755800 \\
$\mathrm{H}$ & 2.60917600 & 1.37100200 & -0.42775800 \\
$\mathrm{H}$ & 2.43265200 & 0.45351000 & 1.06915100 \\
$\mathrm{C}$ & 5.13799700 & 0.78815000 & 0.55731200 \\
$\mathrm{H}$ & 4.21035500 & -1.14857600 & 0.29884600 \\
$\mathrm{H}$ & 4.38929800 & -0.23197500 & -1.19704800
\end{tabular}


SUPPORTING INFORMATION

$\begin{array}{llll}\mathrm{H} & 6.16711300 & 0.41348800 & 0.45110400 \\ \mathrm{H} & 5.10398700 & 1.80218600 & 0.12722500 \\ \mathrm{H} & 4.92333300 & 0.87856700 & 1.63447000\end{array}$

1 imaginary frequency: $-500.7488 \mathrm{~cm}^{-1}$

TC[G] ( $\omega B$ 97X-D/cc-pVDZ at $298.15 \mathrm{~K})=0.193791 \mathrm{au}$ TC[G] ( $\omega B$ 97X-D/cc-pVDZ at $195.15 \mathrm{~K})=0.212025 \mathrm{au}$

$E$ (B2PLYP-D3/ aug-cc-pVTZ in gas phase) $=-639.966756$ au

E (B2PLYP-D3/ aug-cc-pVTZ in THF) $=-639.983858 \mathrm{au}$

$\begin{array}{lrrr}\text { TS }_{\text {trans_3butoxy }} & & \\ \mathrm{C} & -1.63916400 & -0.93930100 & -1.09212300 \\ \mathrm{H} & -1.08531000 & -0.23799200 & -1.72887700 \\ \mathrm{H} & -2.15089300 & -1.68513200 & -1.72157800 \\ \mathrm{C} & -2.11413100 & -1.29113000 & 1.02033700 \\ \mathrm{H} & -2.78852100 & -2.15623100 & 1.11729500 \\ \mathrm{H} & -1.90075300 & -0.86888900 & 2.00886000 \\ \mathrm{C} & -2.65011400 & -0.37175800 & -0.08806500 \\ \mathrm{O} & -3.79001700 & 0.10876800 & -0.27563800 \\ \mathrm{Li} & -3.48404900 & 1.91220900 & -0.14352300 \\ \mathrm{H} & -1.73504300 & 0.92953000 & 0.39801500 \\ \mathrm{Al} & -0.79353300 & 2.32585300 & 0.10066300 \\ \mathrm{H} & -0.12771700 & 2.83539600 & 1.45926500 \\ \mathrm{H} & 0.07557300 & 2.00024600 & -1.20637800 \\ \mathrm{H} & -2.15948200 & 3.23162700 & -0.30511200 \\ \mathrm{C} & -0.89699900 & -1.58838600 & 0.11575900 \\ \mathrm{H} & -0.64428900 & -2.65607300 & -0.01458700 \\ \mathrm{C} & 1.35410000 & -0.99847400 & -0.25670800 \\ \mathrm{C} & 2.47736100 & -0.16955800 & 0.33431200 \\ \mathrm{H} & 1.64922000 & -2.06607400 & -0.32729500 \\ \mathrm{H} & 1.12586400 & -0.64945300 & -1.28300000 \\ \mathrm{C} & 3.76156000 & -0.25136300 & -0.48706300 \\ \mathrm{H} & 2.14111000 & 0.87900400 & 0.40026300 \\ \mathrm{H} & 2.66273400 & -0.50953200 & 1.36718200 \\ \mathrm{H} & 4.08572400 & -1.30462300 & -0.56188600 \\ \mathrm{H} & 3.55593300 & 0.08109500 & -1.51969000 \\ \mathrm{C} & 4.89063600 & 0.58893300 & 0.10213600 \\ \mathrm{H} & 5.13812500 & 0.25919600 & 1.12391500 \\ \mathrm{H} & 5.80526600 & 0.51735000 & -0.50529200 \\ \mathrm{H} & 4.60443100 & 1.65124500 & 0.15706500\end{array}$


SUPPORTING INFORMATION

$\mathrm{O}$

$0.21709200-0.87091600 \quad 0.57217200$

1 imaginary frequency: $-406.9138 \mathrm{~cm}^{-1}$

TC[G] $(\omega B 97 X-D / c c-p V D Z$ at $298.15 \mathrm{~K})=0.197603 \mathrm{au}$

TC[G] $(\omega B 97 X-D / c c-p V D Z$ at $195.15 \mathrm{~K})=0.216249$ au

$E$ (B2PLYP-D3/ aug-cc-pVTZ in gas phase $)=-715.083032$ au

$E(B 2 P L Y P-D 3 /$ aug-cc-pVTZ in THF) $=-715.103289$ au

\section{TS $_{\text {cis_- }} 3$ butoxy}

$\begin{array}{llrr}\text { C } & -0.89996700 & -0.59997300 & -0.96654900 \\ \text { H } & -1.05589900 & -0.00335800 & -1.87264700 \\ \text { H } & -0.34439300 & -1.52122800 & -1.20953500 \\ \mathrm{C} & -1.35923700 & -0.67711300 & 1.17485500 \\ \mathrm{H} & -0.93308100 & -1.61617000 & 1.56372100 \\ \mathrm{H} & -1.87435900 & -0.13653200 & 1.97695600 \\ \mathrm{C} & -2.11250400 & -0.99360900 & -0.11809600 \\ \mathrm{O} & -3.01638200 & -1.83231200 & -0.34102300 \\ \mathrm{Li} & -4.64341600 & -1.08134500 & -0.52678500 \\ \mathrm{H} & -2.83131100 & 0.49127300 & -0.25283100 \\ \mathrm{Al} & -4.06464800 & 1.64943900 & -0.00962300 \\ \mathrm{H} & -4.01280800 & 2.03122000 & 1.54514700 \\ \mathrm{H} & -3.96730300 & 2.75442400 & -1.16133600 \\ \mathrm{H} & -5.33152800 & 0.58560900 & -0.30893800 \\ \mathrm{C} & -0.30659300 & 0.05380000 & 0.31807100 \\ \mathrm{H} & -0.44674000 & 1.14970900 & 0.31997100 \\ \mathrm{C} & 1.96753400 & 0.35382600 & -0.14687300 \\ \mathrm{C} & 3.34784200 & -0.08265100 & 0.30453500 \\ \mathrm{H} & 1.81148300 & 0.09196700 & -1.21373100 \\ \mathrm{H} & 1.86298400 & 1.45542400 & -0.06566600 \\ \mathrm{C} & 4.46707100 & 0.56519200 & -0.50694800 \\ \mathrm{H} & 3.46177500 & 0.16532800 & 1.37333200 \\ \mathrm{H} & 3.41189900 & -1.18136700 & 0.22841500 \\ \mathrm{H} & 4.33390400 & 0.32178400 & -1.57587400 \\ \mathrm{H} & 4.38607000 & 1.66384200 & -0.43151300 \\ \mathrm{C} & 5.85583400 & 0.12471500 & -0.05370900 \\ \mathrm{H} & 6.64527800 & 0.60417200 & -0.65140000 \\ \mathrm{H} & 6.02856700 & 0.38515000 & 1.00277500 \\ \mathrm{H} & 5.97644500 & -0.96621700 & -0.14995100 \\ \mathrm{O} & 1.00260900 & -0.28504100 & 0.66062900\end{array}$




\section{SUPPORTING INFORMATION}

1 imaginary frequency: $-386.0528 \mathrm{~cm}^{-1}$

TC[G] ( $\omega B 97 X-D / c c-p V D Z$ at $298.15 \mathrm{~K})=0.194036 \mathrm{au}$

TC[G] ( $\omega B$ 97X-D/cc-pVDZ at 195.15 K) $=0.0213842 \mathrm{au}$

$E$ (B2PLYP-D3/ aug-cc-pVTZ in gas phase) $=-715.086307$ au

E (B2PLYP-D3/ aug-cc-pVTZ in THF) $=-715.107494$ au

$\begin{array}{crcc}\text { TS }^{\prime} \text { trans_3butoxy } & & \\ \mathrm{C} & 1.22557800 & -1.10638500 & 1.06515100 \\ \mathrm{H} & 0.76702200 & -0.33249700 & 1.69198400 \\ \mathrm{H} & 1.58713000 & -1.92794200 & 1.70396500 \\ \mathrm{C} & 1.66554400 & -1.40553900 & -1.07297300 \\ \mathrm{H} & 2.20219600 & -2.34249500 & -1.28666900 \\ \mathrm{H} & 1.49801500 & -0.85050900 & -2.00259600 \\ \mathrm{C} & 2.34790600 & -0.68801800 & 0.10172000 \\ \mathrm{O} & 3.56480600 & -0.54294100 & 0.33119800 \\ \mathrm{Li} & 4.42270500 & 1.05258900 & 0.50337600 \\ \mathrm{H} & 1.64697600 & 0.79264000 & -0.25582100 \\ \mathrm{Al} & 2.28826600 & 2.33286000 & -0.13801500 \\ \mathrm{H} & 1.31618200 & 3.55543600 & -0.44719800 \\ \mathrm{H} & 2.98181800 & 2.21707500 & 1.36524500 \\ \mathrm{H} & 3.65980900 & 2.12889700 & -1.04674200 \\ \mathrm{C} & 0.42356000 & -1.60511000 & -0.17690400 \\ \mathrm{H} & 0.06248300 & -2.64699000 & -0.09540000 \\ \mathrm{C} & -1.76320200 & -0.83943700 & 0.21299700 \\ \mathrm{C} & -2.79545900 & 0.13732300 & -0.31652200 \\ \mathrm{H} & -2.16373200 & -1.87498200 & 0.19958900 \\ \mathrm{H} & -1.51968600 & -0.60182100 & 1.26852100 \\ \mathrm{C} & -4.09422700 & 0.11613300 & 0.48556900 \\ \mathrm{H} & -2.35821500 & 1.14987400 & -0.30440200 \\ \mathrm{H} & -2.99826400 & -0.10483100 & -1.37344300 \\ \mathrm{H} & -4.51495500 & -0.90500600 & 0.47711200 \\ \mathrm{H} & -3.87630400 & 0.34815900 & 1.54309200 \\ \mathrm{O} & -0.61042500 & -0.75805600 & -0.59353800 \\ \mathrm{C} & -5.13265100 & 1.09907900 & -0.04705800 \\ \mathrm{H} & -5.39404500 & 0.87070100 & -1.09272300 \\ \mathrm{H} & -6.05894500 & 1.06711200 & 0.54579200 \\ \mathrm{H} & -4.75038500 & 2.13192800 & -0.01940600\end{array}$

1 imaginary frequency: $-491.8495 \mathrm{~cm}^{-1}$

TC[G] ( $\omega B$ 97X-D/cc-pVDZ at $298.15 \mathrm{~K})=0.195893 \mathrm{au}$ 


\section{SUPPORTING INFORMATION}

TC[G] ( $\omega B$ 97X-D/cc-pVDZ at $195.15 \mathrm{~K})=0.215199 \mathrm{au}$

$E$ (B2PLYP-D3/ aug-cc-pVTZ in gas phase $)=-715.084273$ au

$E(B 2 P L Y P-D 3 /$ aug-cc-pVTZ in THF) $=-715.103804$ au

\section{TS' ${ }_{\text {cis_-3butoxy }}$}

$\begin{array}{lrrr}\text { C } & 1.28447600 & -0.80719600 & 1.14767700 \\ \text { H } & 1.74162500 & -0.49322300 & 2.09255100 \\ \text { H } & 0.88815000 & -1.83059100 & 1.24452500 \\ \text { C } & 0.89685100 & -0.15971900 & -0.91083400 \\ \text { H } & 0.39284600 & -0.99552700 & -1.42371400 \\ \text { H } & 1.05429700 & 0.66963400 & -1.61007700 \\ \mathrm{C} & 2.10173500 & -0.73092400 & -0.14906900 \\ \mathrm{O} & 3.00428600 & -1.49576300 & -0.55978900 \\ \mathrm{Li} & 4.75060700 & -1.08682600 & -0.76769000 \\ \mathrm{H} & 2.71012500 & 0.72152500 & 0.19181300 \\ \mathrm{Al} & 4.28636200 & 1.31657000 & 0.06994300 \\ \mathrm{H} & 4.49352300 & 2.83432100 & 0.50151000 \\ \mathrm{H} & 5.07219400 & 0.11151000 & 0.88158500 \\ \mathrm{H} & 4.61079000 & 0.84736700 & -1.48050600 \\ \mathrm{C} & 0.22984900 & 0.09277900 & 0.47406800 \\ \mathrm{H} & 0.31467900 & 1.15077500 & 0.78214500 \\ \mathrm{C} & -2.03681400 & 0.41832600 & -0.01040900 \\ \mathrm{C} & -3.41057700 & -0.17452800 & 0.23715800 \\ \mathrm{H} & -1.99288200 & 1.46305400 & 0.36170000 \\ \mathrm{H} & -1.82474700 & 0.45438000 & -1.09892600 \\ \mathrm{C} & -4.52730100 & 0.61918300 & -0.43640300 \\ \mathrm{H} & -3.41432000 & -1.21632500 & -0.12579200 \\ \mathrm{H} & -3.58166000 & -0.22087400 & 1.32596200 \\ \mathrm{H} & -4.50622300 & 1.66206700 & -0.07395600 \\ \mathrm{H} & -4.33771800 & 0.66977000 & -1.52317700 \\ \mathrm{O} & -1.07485800 & -0.37301500 & 0.65065700 \\ \mathrm{C} & -5.90917200 & 0.02175600 & -0.18805400 \\ \mathrm{H} & -6.13860700 & -0.01087300 & 0.88905800 \\ \mathrm{H} & -6.69701200 & 0.60991300 & -0.68182000 \\ \mathrm{H} & -5.96930200 & -1.00967000 & -0.57081600\end{array}$

1 imaginary frequency: $-500.7441 \mathrm{~cm}^{-1}$

TC[G] ( $\omega B$ 97X-D/cc-pVDZ at $298.15 \mathrm{~K})=0.195905 \mathrm{au}$ TC[G] $(\omega B 97 X-D / c c-p V D Z$ at $195.15 \mathrm{~K})=0.215209 \mathrm{au}$ $E(B 2 P L Y P-D 3 /$ aug-cc-pVTZ in gas phase $)=-715.086782$ au 
SUPPORTING INFORMATION

E (B2PLYP-D3/ aug-cc-pVTZ in THF) $=-715.105711$ au

$\begin{array}{lrrr}\text { TS }_{\text {trans_3tbutyl }} & & \\ \mathrm{C} & -0.01598600 & -1.24096900 & -1.03293100 \\ \mathrm{H} & -0.07313300 & -0.49799800 & -1.83763200 \\ \mathrm{H} & 0.06147100 & -2.24315300 & -1.47933800 \\ \mathrm{C} & -0.16343500 & -0.91146500 & 1.15468600 \\ \mathrm{H} & -0.16414800 & -1.73637900 & 1.88218900 \\ \mathrm{H} & -0.31427300 & 0.03412800 & 1.69031500 \\ \mathrm{C} & -1.14111700 & -1.19970200 & 0.00952800 \\ \mathrm{O} & -2.18517500 & -1.89740500 & 0.04553800 \\ \mathrm{Li} & -3.64446600 & -0.84701700 & -0.07923600 \\ \mathrm{H} & -1.64405800 & 0.33582400 & -0.29222000 \\ \mathrm{Al} & -2.58054300 & 1.74618300 & -0.04591400 \\ \mathrm{H} & -2.17949400 & 2.27452400 & 1.41317400 \\ \mathrm{H} & -2.48172400 & 2.67448300 & -1.34374800 \\ \mathrm{H} & -4.05204700 & 0.93347500 & 0.00529000 \\ \mathrm{C} & 0.99839900 & -0.95142300 & 0.11524700 \\ \mathrm{H} & 1.64286400 & -1.82678500 & 0.28965500 \\ \mathrm{C} & 1.92021500 & 0.27855500 & -0.00769300 \\ \mathrm{C} & 2.87928800 & 0.04322400 & -1.18324900 \\ \mathrm{C} & 1.14569400 & 1.58198000 & -0.24243300 \\ \mathrm{C} & 2.72780900 & 0.40267700 & 1.29212300 \\ \mathrm{H} & 3.46531900 & -0.87918500 & -1.04066300 \\ \mathrm{H} & 2.33299400 & -0.04716600 & -2.13566200 \\ \mathrm{H} & 3.58752400 & 0.88062800 & -1.28430100 \\ \mathrm{H} & 0.46954200 & 1.81591000 & 0.59464100 \\ \mathrm{H} & 1.84735000 & 2.42514100 & -0.33969100 \\ \mathrm{H} & 0.54560700 & 1.54794400 & -1.16496500 \\ \mathrm{H} & 3.42582000 & 1.25302700 & 1.23962900 \\ \mathrm{H} & 2.06848700 & 0.56623400 & 2.15934500 \\ \mathrm{H} & 3.31855000 & -0.50798600 & 1.48259200\end{array}$

1 imaginary frequency: $-386.2853 \mathrm{~cm}^{-1}$

TC[G] $(\omega B 97 X-D / c c-p V D Z$ at $298.15 \mathrm{~K})=0.193277 \mathrm{au}$ TC[G] ( $\omega B$ 97X-D/cc-pVDZ at $195.15 \mathrm{~K})=0.210990 \mathrm{au}$ $E$ (B2PLYP-D3/ aug-cc-pVTZ in gas phase) $=-639.963226$ au E (B2PLYP-D3/ aug-cc-pVTZ in THF) $=-639.981017$ au

TS $_{\text {cis__3tbutyl }}$ 


\section{SUPPORTING INFORMATION}

$\begin{array}{llrr}\text { C } & 0.22607200 & 0.37495400 & -1.06140700 \\ \mathrm{H} & 0.69125900 & -0.30999500 & -1.78084300 \\ \mathrm{H} & -0.29751700 & 1.17684400 & -1.60748900 \\ \mathrm{C} & 0.06821400 & 0.77725800 & 1.08496800 \\ \mathrm{H} & -0.50581400 & 1.70844800 & 1.22199700 \\ \mathrm{H} & 0.40494500 & 0.41476400 & 2.06336600 \\ \mathrm{C} & 1.11021200 & 1.04169000 & -0.00565100 \\ \mathrm{O} & 1.93655400 & 1.97830100 & -0.12286700 \\ \mathrm{Li} & 3.62929100 & 1.36017900 & -0.06539100 \\ \mathrm{H} & 1.99914200 & -0.30082900 & 0.35277800 \\ \mathrm{Al} & 3.25084200 & -1.43142500 & 0.06760800 \\ \mathrm{H} & 3.49854900 & -2.28499000 & 1.39659600 \\ \mathrm{H} & 2.90216300 & -2.12122800 & -1.33586100 \\ \mathrm{H} & 4.44861400 & -0.26818400 & -0.12961300 \\ \mathrm{C} & -0.62001500 & -0.21164600 & 0.10279900 \\ \mathrm{H} & -0.26295900 & -1.23092900 & 0.31900400 \\ \mathrm{C} & -2.15327200 & -0.26502600 & -0.00073700 \\ \mathrm{C} & -2.70626400 & -0.78666100 & 1.33303800 \\ \mathrm{C} & -2.77705800 & 1.10523400 & -0.29887100 \\ \mathrm{C} & -2.52314600 & -1.23929300 & -1.12805000 \\ \mathrm{H} & -2.29189800 & -1.77880200 & 1.57362300 \\ \mathrm{H} & -2.45490900 & -0.10591000 & 2.16229700 \\ \mathrm{H} & -3.80334300 & -0.87875600 & 1.29564100 \\ \mathrm{H} & -2.42688100 & 1.52010300 & -1.25669100 \\ \mathrm{H} & -3.87256600 & 1.01366500 & -0.36564500 \\ \mathrm{H} & -2.56024900 & 1.84001800 & 0.49195500 \\ \mathrm{H} & -3.61629300 & -1.34284700 & -1.21620200 \\ \mathrm{H} & -2.13963600 & -0.88856300 & -2.09959400 \\ \mathrm{H} & -2.10231800 & -2.24027700 & -0.94165700\end{array}$

1 imaginary frequency: $-393.2560 \mathrm{~cm}^{-1}$

TC[G] $(\omega B 97 X-D / c c-p V D Z$ at $298.15 \mathrm{~K})=0.192358 \mathrm{au}$ TC[G] $(\omega B 97 X-D / c c-p V D Z$ at $195.15 \mathrm{~K})=0.210417 \mathrm{au}$

$E$ (B2PLYP-D3/ aug-cc-pVTZ in gas phase) $=-639.965455$ au E (B2PLYP-D3/ aug-cc-pVTZ in THF) $=-639.983608$ au

TS' ${ }_{\text {trans_3 } 3 \text { tbutyl }}$
C
$0.05773300-0.96405000$
1.10870400
$\mathrm{H}$
$0.09709300-0.12175200 \quad 1.80992400$
$\mathrm{H}$
$0.08247200-1.90212500 \quad 1.68227000$ 


\section{SUPPORTING INFORMATION}

$\mathrm{C}$

$0.05815500 \quad-0.96318700-1.10895200$

$\mathrm{H} \quad 0.08310300 \quad-1.90082300 \quad-1.68322700$

H $\quad \begin{array}{llll}0.09778900 & -0.12035600 & -1.80951500\end{array}$

$\begin{array}{llll}\text { C } & 1.12198600 & -0.98652500 & 0.00007200\end{array}$

$\begin{array}{lllll}\mathrm{O} & 2.20445900 & -1.61658100 & 0.00003800\end{array}$

Li $\quad \begin{array}{llll}3.85923900 & -0.88676100 & -0.00071700\end{array}$

$\begin{array}{llll}\mathrm{H} & 1.40656400 & 0.61962700 & 0.00082000\end{array}$

$\begin{array}{lllll}\text { Al } & 2.84708100 & 1.48624700 & 0.00023600\end{array}$

$\begin{array}{llll}\mathrm{H} & 2.72004300 & 3.07359300 & 0.00056600\end{array}$

H $\quad 3.60795300 \quad 0.72415300 \quad 1.25654300$

H $\quad 3.60663500 \quad 0.72463800 \quad-1.25716900$

C $\quad-1.03711200-0.91155000-0.00031100$

$\mathrm{H} \quad-1.63287200 \quad-1.83744300-0.00078500$

C $\quad-2.03187600 \quad 0.26722900 \quad-0.00001500$

C $\quad \begin{array}{llll}-2.91259700 & 0.15121300 & 1.25226000\end{array}$

C $\quad-1.33649800 \quad 1.63473500 \quad 0.00065200$

C $\quad-2.91218000 \quad 0.15222000 \quad-1.25267400$

$\mathrm{H} \quad \begin{array}{llll}-3.44616700 & -0.81279100 & 1.27545900\end{array}$

$\mathrm{H} \quad \begin{array}{llll}\mathrm{H} & -2.31272300 & 0.22722400 & 2.17309700\end{array}$

$\begin{array}{llll}\mathrm{H} & -3.66585900 & 0.95450900 & 1.27785000\end{array}$

$\mathrm{H} \quad-0.70181000 \quad 1.77554000 \quad-0.88740500$

$\begin{array}{llll}\mathrm{H} & -2.08820400 & 2.43972200 & 0.00095200\end{array}$

$\begin{array}{llll}\mathrm{H} & -0.70194700 & 1.77474200 & 0.88893200\end{array}$

$\mathrm{H} \quad-3.66539700 \quad 0.95557100 \quad-1.27789700$

$\mathrm{H} \quad-2.31199200 \quad 0.22891600 \quad-2.17325000$

H $\quad-3.44578700 \quad-0.81174100 \quad-1.27679600$

1 imaginary frequency: $-477.0276 \mathrm{~cm}^{-1}$

TC[G] ( $\omega B$ 97X-D/cc-pVDZ at 298.15 K) $=0.194027$ au

TC[G] $(\omega B 97 X-D / c c-p V D Z$ at $195.15 \mathrm{~K})=0.211687 \mathrm{au}$

$E$ (B2PLYP-D3/ aug-cc-pVTZ in gas phase) $=-639.963478$ au

E (B2PLYP-D3/ aug-cc-pVTZ in THF) $=-639.979594$ au

TS' ${ }_{\text {cis__ }}$ 3tbutyl

$\begin{array}{lrrr}\mathrm{C} & 0.12482600 & 0.37743800 & -1.09548400 \\ \mathrm{H} & 0.46827300 & -0.20012100 & -1.96189700 \\ \mathrm{H} & -0.33336400 & 1.31618300 & -1.44696600 \\ \mathrm{C} & 0.12483000 & 0.37742000 & 1.09547500 \\ \mathrm{H} & -0.33336000 & 1.31615900 & 1.44697500 \\ \mathrm{H} & 0.46828300 & -0.20015200 & 1.96187700\end{array}$




\section{SUPPORTING INFORMATION}

$\begin{array}{llll}\text { C } & 1.13583300 & 0.74240400 & -0.00000300 \\ \text { O } & 2.00869800 & 1.64056600 & 0.00000200 \\ \text { Li } & 3.79743900 & 1.38919300 & 0.00002500 \\ \text { H } & 1.82789800 & -0.72156300 & -0.00001700 \\ \text { Al } & 3.45271700 & -1.17094800 & -0.00000400 \\ \text { H } & 3.74937800 & -2.73492400 & -0.00000600 \\ \text { H } & 3.97708300 & -0.23505700 & -1.25757900 \\ \text { H } & 3.97705700 & -0.23507400 & 1.25759600 \\ \text { C } & -0.72188400 & -0.32872400 & -0.00000800 \\ \text { H } & -0.47787000 & -1.40288900 & -0.00001800 \\ \text { C } & -2.25457700 & -0.19917400 & 0.00000000 \\ \text { C } & -2.79605200 & -0.89936400 & 1.25432900 \\ \text { C } & -2.72710400 & 1.26113600 & 0.00003200 \\ \text { C } & -2.79606400 & -0.89931700 & -1.25434900 \\ \text { H } & -2.48992600 & -1.95752700 & 1.28143700 \\ \text { H } & -2.42281200 & -0.41684400 & 2.17191200 \\ \text { H } & -3.89687700 & -0.86544400 & 1.28109800 \\ \text { H } & -2.38117000 & 1.80744600 & -0.89102000 \\ \text { H } & -3.82781600 & 1.30135100 & 0.00002500 \\ \text { H } & -2.38118200 & 1.80740200 & 0.89111500 \\ \text { H } & -3.89689000 & -0.86541400 & -1.28109600 \\ \text { H } & -2.42284900 & -0.41675200 & -2.17191700 \\ \text { H } & -2.48992300 & -1.95747500 & -1.28150800\end{array}$

1 imaginary frequency: $-504.0093 \mathrm{~cm}^{-1}$

TC[G] $(\omega B 97 X-D / c c-p V D Z$ at $298.15 \mathrm{~K})=0.193874 \mathrm{au}$

TC[G] ( $\omega B$ 97X-D/cc-pVDZ at $195.15 \mathrm{~K})=0.211571 \mathrm{au}$

$E$ (B2PLYP-D3/ aug-cc-pVTZ in gas phase) $=-639.966005$ au

E (B2PLYP-D3/ aug-cc-pVTZ in THF) $=-639.982092$ au

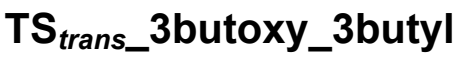

$\begin{array}{lrrr}\mathrm{C} & 0.50811600 & -1.63886900 & -1.26246400 \\ \mathrm{H} & -0.01164800 & -2.21497300 & -2.04477800 \\ \mathrm{H} & 1.29995200 & -1.03368100 & -1.72007400 \\ \mathrm{C} & -0.27147800 & -2.09470300 & 0.74081900 \\ \mathrm{H} & -0.07302900 & -1.79985500 & 1.77702800 \\ \mathrm{H} & -1.07916900 & -2.84319100 & 0.70823300 \\ \mathrm{C} & -0.48046500 & -0.93841000 & -0.27443600 \\ \mathrm{C} & 0.89404000 & -2.58758700 & -0.12461100 \\ \mathrm{C} & -1.90400300 & -0.73596700 & -0.78254300\end{array}$




\section{SUPPORTING INFORMATION}

C $\begin{array}{lll}-2.84943300 & -0.18725800 & 0.28175900\end{array}$

$\mathrm{H} \quad-2.27709100 \quad-1.70549100 \quad-1.15496100$

H $\quad-1.89009200 \quad-0.06456500 \quad-1.65891400$

$\begin{array}{lllll}\text { C } & -4.27239600 & 0.02419400 & -0.22927100\end{array}$

$\begin{array}{llll}\mathrm{H} & -2.86717800 & -0.87735600 & 1.14478600\end{array}$

$\begin{array}{llll}\mathrm{H} & -2.44706600 & 0.76364900 & 0.66947300\end{array}$

C $\quad-5.21194200 \quad 0.57634500 \quad 0.83835200$

$\mathrm{H} \quad-4.24841400 \quad 0.71207400 \quad-1.09302100$

$\mathrm{H} \quad-4.66902500 \quad-0.93212000-0.61389100$

$\mathrm{H} \quad-6.22901300 \quad 0.72317100 \quad 0.44514700$

$\mathrm{H} \quad \begin{array}{llll}-5.28018200 & -0.10841500 & 1.69903100\end{array}$

$\begin{array}{llll}\mathrm{H} & -4.85388800 & 1.54744300 & 1.21642300\end{array}$

C $\quad 0.21230800 \quad 1.34684400 \quad-0.53449300$

$\begin{array}{llll}\text { C } & 0.96055100 & 2.42308900 & 0.22854800\end{array}$

$\begin{array}{lllll}\mathrm{H} & 0.77327400 & 1.06960400 & -1.44794100\end{array}$

$\mathrm{H} \quad-0.77586500 \quad 1.73118000 \quad-0.85495000$

C $\quad 1.17614100 \quad 3.68587200 \quad-0.60141000$

$\begin{array}{llll}\mathrm{H} & 0.39741100 & 2.66169400 & 1.14674900\end{array}$

$\begin{array}{llll}\mathrm{H} & 1.93449100 & 2.01846500 & 0.55076000\end{array}$

$\mathrm{H} \quad 1.73496900 \quad 3.42761700 \quad-1.51800500$

$\mathrm{H} \quad 0.20009200 \quad 4.07993000 \quad-0.93659100$

$\begin{array}{lllll}\text { C } & 1.92860800 & 4.77039100 & 0.16383100\end{array}$

$\begin{array}{llll}\mathrm{H} & 2.07707700 & 5.66949300 & -0.45283600\end{array}$

$\begin{array}{llll}\mathrm{H} & 1.37805000 & 5.07042500 & 1.06990900\end{array}$

$\begin{array}{llll}\mathrm{H} & 2.92033400 & 4.41193100 & 0.48223200\end{array}$

$\begin{array}{lllll}\mathrm{O} & 0.06172000 & 0.22734000 & 0.31598100\end{array}$

$\begin{array}{lllll}0 & 1.44336300 & -3.71325500 & -0.17625800\end{array}$

Li $\quad 3.16035700 \quad-3.38388800 \quad 0.34228500$

H $\quad 2.02073500 \quad-1.61167300 \quad 0.59732700$

Al $\quad 3.44757600 \quad-0.66932900 \quad 0.58076100$

$\mathrm{H} \quad 4.43810400 \quad-2.02668800 \quad 0.44385600$

$\begin{array}{llll}\mathrm{H} & 3.64003800 & 0.06355900 & 1.98641200\end{array}$

$\begin{array}{llll}\mathrm{H} & 3.40663600 & 0.14439500 & -0.80042100\end{array}$

1 imaginary frequency: $-416.7726 \mathrm{~cm}^{-1}$

TC[G] ( $\omega B 97 X-D / c c-p V D Z$ at $298.15 \mathrm{~K})=0.303519 \mathrm{au}$

TC[G] $(\omega B 97 X-D / c c-p V D Z$ at $195.15 \mathrm{~K})=0.326042 \mathrm{au}$

$E(B 2 P L Y P-D 3 /$ aug-cc-pVTZ in gas phase $)=-872.019414$ au

E (B2PLYP-D3/ aug-cc-pVTZ in THF) $=-872.041890$ au 


\section{SUPPORTING INFORMATION}

TS cis_3butoxy_3butyl

$\begin{array}{lrrr}\text { C } & -1.17961100 & -0.82758600 & 1.21674200 \\ \text { H } & -0.63264300 & -1.30421300 & 2.04374600 \\ \text { H } & -1.88551500 & -0.08598100 & 1.61003100 \\ \mathrm{C} & -0.60705900 & -1.65250400 & -0.74257000 \\ \mathrm{H} & -0.88947800 & -1.51843300 & -1.79376400 \\ \mathrm{H} & 0.13929400 & -2.45704200 & -0.65202200 \\ \mathrm{C} & -0.19862500 & -0.39575800 & 0.09495100 \\ \mathrm{C} & -1.71648300 & -1.91160300 & 0.28198800 \\ \mathrm{C} & 2.14407000 & -0.30223800 & -0.35894300 \\ \mathrm{C} & 3.45822300 & -0.69659000 & 0.28898600 \\ \mathrm{H} & 1.94298800 & -0.94565200 & -1.23951300 \\ \mathrm{H} & 2.20310100 & 0.74150900 & -0.72486200 \\ \mathrm{C} & 4.65018500 & -0.54701800 & -0.65295300 \\ \mathrm{H} & 3.60539300 & -0.07339900 & 1.18738500 \\ \mathrm{H} & 3.38010900 & -1.73925600 & 0.64082400 \\ \mathrm{H} & 4.48488600 & -1.16206100 & -1.55526700 \\ \mathrm{H} & 4.71176500 & 0.49835000 & -1.00345200 \\ \mathrm{C} & -0.28100800 & 2.14681300 & 0.32065600 \\ \mathrm{C} & -0.64121500 & 3.46747600 & -0.35550300 \\ \mathrm{H} & -0.86772500 & 2.04306700 & 1.25059200 \\ \mathrm{H} & 0.77707500 & 2.16235500 & 0.63297400 \\ \mathrm{H} & -0.05982200 & 3.56833300 & -1.28925700 \\ \mathrm{H} & -1.70259900 & 3.44181700 & -0.65822200 \\ \mathrm{O} & -2.28892200 & -2.99099500 & 0.56975000 \\ \mathrm{Li} & -4.05882800 & -2.90663900 & 0.24893600 \\ \mathrm{H} & -2.86156000 & -1.00247900 & -0.47860000 \\ \mathrm{Al} & -4.38453500 & -0.22553700 & -0.55982500 \\ \mathrm{H} & -5.26758300 & -1.59373300 & -0.14830300 \\ \mathrm{H} & -4.66421600 & 0.21165100 & -2.07199100 \\ \mathrm{H} & -4.38099100 & 0.83461800 & 0.64201000 \\ \mathrm{C} & -0.52995000 & 0.93655900 & -0.57320300 \\ \mathrm{H} & -1.58624400 & 0.91198800 & -0.88631100 \\ \mathrm{H} & 0.05456400 & 1.02901200 & -1.50562800 \\ \mathrm{H} & -0.39153700 & 4.67943000 & 0.53660400 \\ \mathrm{H} & 0.66861600 & 4.74385800 & 0.83090200 \\ \mathrm{H} & -0.65624900 & 5.61777300 & 0.02659900 \\ & 5.97870700 & 4.61996100 & 1.46074100 \\ \mathrm{H} & 5.95042900 & -1.99498800 & 0.32879000\end{array}$




\section{SUPPORTING INFORMATION}
$\mathrm{H}$
$6.81519500-0.82759200-0.69791400$
$\mathrm{H}$
$6.17834400-0.32272100 \quad 0.88461700$
$\mathrm{O}$
$1.11839100 \quad-0.44180000 \quad 0.60085400$

1 imaginary frequency: $-407.3471 \mathrm{~cm}^{-1}$

TC[G] $(\omega B 97 X-D / c c-p V D Z$ at $298.15 \mathrm{~K})=0.301067 \mathrm{au}$ TC[G] $(\omega B 97 X-D / c c-p V D Z$ at $195.15 \mathrm{~K})=0.324386 \mathrm{au}$

$E(B 2 P L Y P-D 3 /$ aug-cc-pVTZ in gas phase $)=-872.020888$ au

$E$ (B2PLYP-D3/ aug-cc-pVTZ in THF) $=-872.043770 \mathrm{au}$

\section{TS' ${ }_{\text {trans_3butoxy_3butyl }}$}

C

$\begin{array}{lll}-0.79144000 & -1.21358000 & 1.30753000\end{array}$

$\mathrm{H}$

$\begin{array}{lll}-0.43432200 & -1.85494800 & 2.12925100\end{array}$

$\mathrm{H}$

$\begin{array}{lll}-1.39689400 & -0.39946100 & 1.72177700\end{array}$

C

$-0.18409200-1.94453100-0.67832700$

$\mathrm{H}$

$-0.32743000 \quad-1.63371700-1.71864400$

$\mathrm{H}$

$\begin{array}{llll}0.40573000 & -2.87398500 & -0.63936700\end{array}$

C

$\begin{array}{lll}0.33887800 & -0.84938400 & 0.28934600\end{array}$

C

$\begin{array}{lll}-1.41687000 & -2.09670300 & 0.22018200\end{array}$

C

$2.81481200-0.80397300-0.30182800$

C

$\begin{array}{lll}4.24913600 & -0.94083000 & 0.20280300\end{array}$

$\mathrm{H}$

$2.64118100-1.52973500-1.11686100$

$\mathrm{H}$

2.67159800

0.19174300

$-0.75415600$

$\mathrm{H}$

$\begin{array}{lll}4.41728200 & -0.21451700 & 1.01771800\end{array}$

$\mathrm{H}$

$4.38446700-1.93978000$

0.65398800

C

$0.29135300 \quad 1.54205200$

0.45761600

C

$-0.39627700$

$2.70601100-0.23189900$

$\mathrm{H}$

$\begin{array}{lll}-0.14602200 & 1.38494900 & 1.46311700\end{array}$

$\mathrm{H}$

1.36720300

1.76550200

0.60342800

$\mathrm{H}$

0.02199400

2.80772400

$-1.24763700$

$\mathrm{H}$

$-1.46406000$

$2.45874900-0.35710300$

$\mathrm{O}$

$-2.17633300-3.07869600$

0.34909800

$\mathrm{Li}$

$-3.98792800$

$-3.01661600$

0.17389800

$\mathrm{H}$

$-2.24094500-0.83255500$

$-0.50915300$

$\mathrm{H}$

$\begin{array}{lll}-4.36547800 & 0.80116700 & -1.27472300\end{array}$

$\mathrm{H}$

$-4.41168800-1.10687300$

0.77328800

$\mathrm{H}$

$-4.23969800$

$-1.56575800$

Al

$-3.88272400$

$-1.99285900$

$-0.72077900$

C

$-0.24489400$

4.01934000

0.53111100

$\mathrm{H}$

$-0.65266400$

3.90072500

1.55072300 


\section{SUPPORTING INFORMATION}

$\begin{array}{lrrr}\text { H } & 0.82778300 & 4.25101000 & 0.65628400 \\ \mathrm{C} & -0.94110600 & 5.18703900 & -0.16177700 \\ \mathrm{H} & -0.82039900 & 6.12229100 & 0.40510400 \\ \mathrm{H} & -0.53048900 & 5.34951400 & -1.17118500 \\ \mathrm{H} & -2.02039600 & 4.99524500 & -0.27075600 \\ \mathrm{O} & 0.12547600 & 0.39472500 & -0.34703800 \\ \mathrm{C} & 1.76989700 & -1.01322500 & 0.79011800 \\ \mathrm{H} & 1.87306200 & -2.02305100 & 1.22296200 \\ \mathrm{H} & 1.94805300 & -0.30944900 & 1.62254200 \\ \mathrm{C} & 5.28847100 & -0.72885100 & -0.89372600 \\ \mathrm{H} & 6.31296000 & -0.82839800 & -0.50488900 \\ \mathrm{H} & 5.16421400 & -1.46401700 & -1.70517400 \\ \mathrm{H} & 5.19380700 & 0.27432400 & -1.33971600\end{array}$

1 imaginary frequency: $-497.0056 \mathrm{~cm}^{-1}$

TC[G] ( $\omega B$ 97X-D/cc-pVDZ at 298.15 K) $=0.302706$ au TC[G] $(\omega B 97 X-D / c c-p V D Z$ at $195.15 \mathrm{~K})=0.325602 \mathrm{au}$ $E$ (B2PLYP-D3/ aug-cc-pVTZ in gas phase) $=-872.020019$ au E (B2PLYP-D3/ aug-cc-pVTZ in THF) $=-872.041560 \mathrm{au}$

\section{TS' ${ }_{\text {cis_3butoxy_3butyl }}$}

$\begin{array}{lrrr}\text { C } & -1.25330500 & -0.51408100 & 1.25942600 \\ \text { H } & -0.76633100 & -1.05390400 & 2.08475700 \\ \text { H } & -1.84152300 & 0.32171000 & 1.65630300 \\ \text { C } & -0.81441100 & -1.43575700 & -0.69174300 \\ \text { H } & -1.07925500 & -1.28219600 & -1.74487300 \\ \text { H } & -0.18226800 & -2.33130000 & -0.58992800 \\ \mathrm{C} & -0.23469800 & -0.23395900 & 0.12339900 \\ \mathrm{C} & -1.94702500 & -1.52659800 & 0.34040400 \\ \mathrm{C} & 2.09219500 & -0.46753300 & -0.35733600 \\ \mathrm{C} & 3.35151500 & -1.01978500 & 0.28426200 \\ \mathrm{H} & 1.79362900 & -1.09813300 & -1.21912000 \\ \mathrm{H} & 2.28584500 & 0.54997700 & -0.75058200 \\ \mathrm{H} & 3.59433800 & -0.40229400 & 1.16572700 \\ \mathrm{H} & 3.13988600 & -2.03452200 & 0.66183800 \\ \mathrm{C} & 0.04981300 & 2.29763900 & 0.30335400 \\ \mathrm{C} & -0.11339000 & 3.64611000 & -0.39402800 \\ \mathrm{H} & -0.52945000 & 2.30059900 & 1.24395400 \\ \mathrm{H} & 1.10234400 & 2.15778300 & 0.60264600 \\ \mathrm{H} & 0.46456200 & 3.64004200 & -1.33522600\end{array}$




\section{SUPPORTING INFORMATION}

$\begin{array}{lrrr}\text { H } & -1.16948000 & 3.77939800 & -0.68734400 \\ \text { O } & -2.62399800 & -2.53047000 & 0.65993100 \\ \text { Li } & -4.41380800 & -2.71894900 & 0.49071200 \\ \text { H } & -2.89541900 & -0.43523100 & -0.39839100 \\ \text { H } & -5.13561400 & 0.89319800 & -1.36854600 \\ \text { H } & -5.05872300 & -0.79490400 & 0.87406400 \\ \text { H } & -4.73189600 & -1.88841000 & -1.36798600 \\ \text { Al } & -4.56043800 & -0.40795900 & -0.65367500 \\ \text { C } & 4.53847600 & -1.05222400 & -0.67513700 \\ \text { H } & 4.27843800 & -1.65861100 & -1.56080800 \\ \text { H } & 4.73455000 & -0.03238400 & -1.05066700 \\ \mathrm{C} & 5.80441000 & -1.60936300 & -0.03080400 \\ \text { H } & 5.64741900 & -2.64062200 & 0.32392500 \\ \text { H } & 6.64549800 & -1.62232800 & -0.73996100 \\ \text { H } & 6.10611000 & -1.00278100 & 0.83808300 \\ \mathrm{C} & -0.39265300 & 1.12199500 & -0.56225100 \\ \text { H } & -1.45037300 & 1.23979400 & -0.84925100 \\ \text { H } & 0.17677300 & 1.11726200 & -1.50858800 \\ \mathrm{C} & 0.33095600 & 4.82124900 & 0.47138000 \\ \mathrm{H} & 1.39236200 & 4.72717000 & 0.75262400 \\ \mathrm{H} & 0.20624400 & 5.78017000 & -0.05364600 \\ \mathrm{H} & -0.25470500 & 4.87045500 & 1.40356100 \\ \mathrm{O} & 1.07185500 & -0.44525100 & 0.61725800\end{array}$

1 imaginary frequency: $-510.1098 \mathrm{~cm}^{-1}$

TC[G] $(\omega B 97 X-D / c c-p V D Z$ at $298.15 \mathrm{~K})=0.302440 \mathrm{au}$

TC[G] ( $\omega B$ 97X-D/cc-pVDZ at $195.15 \mathrm{~K})=0.325436 \mathrm{au}$

$E$ (B2PLYP-D3/ aug-cc-pVTZ in gas phase $)=-872.021620$ au

E (B2PLYP-D3/ aug-cc-pVTZ in THF) $=-872.042690$ au

\section{TS trans_3butoxy_3tbutyl}

$\begin{array}{lrrr}\mathrm{C} & -1.17439700 & 0.75011600 & 1.01563300 \\ \mathrm{H} & -2.04820000 & 0.64142900 & 1.67607600 \\ \mathrm{H} & -0.27405100 & 0.86155500 & 1.63099100 \\ \mathrm{C} & -1.68708100 & 0.80104100 & -1.12396000 \\ \mathrm{H} & -1.11283000 & 0.90764700 & -2.05017400 \\ \mathrm{H} & -2.75974000 & 0.74106300 & -1.34880600 \\ \mathrm{C} & -1.14311300 & -0.29032000 & -0.15225600 \\ \mathrm{C} & -1.47897700 & 1.83628300 & -0.01584600 \\ \mathrm{C} & 1.14750300 & -1.09898900 & 0.22529600\end{array}$




\section{SUPPORTING INFORMATION}

C

$2.51051500 \quad-0.84148700 \quad-0.39069300$

$\mathrm{H}$

$\begin{array}{lll}1.09569200 & -0.65328200 & 1.23628200\end{array}$

$\mathrm{H}$

$0.99780300-2.18954400$

0.33383300

C

$3.65180400-1.37480800$

0.47052900

$\mathrm{H}$

$2.53863100-1.30381100-1.39206300$

$\mathrm{H}$

$\begin{array}{llll}2.63850200 & 0.24245700 & -0.54435600\end{array}$

$\mathrm{H}$

$\begin{array}{lll}3.60553200 & -0.90024400 & 1.46638000\end{array}$

$\mathrm{H}$

$3.51615700-2.45836500$

0.63777000

C

$5.02234200-1.12038400-0.14978700$

$\mathrm{H}$

$5.83148000-1.50569800$

0.48859800

$\mathrm{H}$

$5.10869400-1.60759700$

$-1.13434100$

$\mathrm{H}$

$5.19255500-0.04210200-0.29726300$

$\mathrm{O}$

$0.17021900-0.53894800-0.62772400$

O

$-2.05980000 \quad 2.93651100$

0.15409000

$\mathrm{Li}$

$-0.79259700$

4.22557100

0.09174400

$\mathrm{H}$

$\begin{array}{lll}0.07672600 & 2.23407800 & -0.41371700\end{array}$

Al

$\begin{array}{llll}1.60787200 & 2.85893300 & 0.02779400\end{array}$

$\mathrm{H}$

1.02169200

4.40262500

0.35107800

$\mathrm{H}$

$\begin{array}{llll}2.58807400 & 2.86348000 & -1.23391300\end{array}$

$\mathrm{H}$

$\begin{array}{lll}1.99262000 & 2.10074900 & 1.38828600\end{array}$

C

$\begin{array}{lll}-1.99032500 & -1.58503100 & 0.03752400\end{array}$

C

$-1.70058200-2.54520700-1.12385500$

C

$-1.66802300 \quad-2.26627000 \quad 1.38027600$

C

$-3.49696500 \quad-1.26874600 \quad 0.04291500$

$\mathrm{H}$

$-1.88062400-2.05594300-2.09400400$

$\mathrm{H}$

$-0.65814700-2.89179500-1.11775400$

$\mathrm{H}$

$-2.35738100-3.42718600-1.06118800$

$\mathrm{H}$

$\begin{array}{lll}-1.92213800 & -1.61673700 & 2.23265000\end{array}$

$\mathrm{H}$

$-2.26263200 \quad-3.18791000 \quad 1.48237800$

$\mathrm{H}$

$-0.61190300-2.54628000$

1.47560200

$\mathrm{H}$

$\begin{array}{lll}-4.06031700 & -2.16530400 & 0.34411500\end{array}$

$\mathrm{H}$

$-3.75685400-0.46770000$

0.75317000

$\mathrm{H}$

$\begin{array}{lll}-3.86344800 & -0.97612500 & -0.95177000\end{array}$

1 imaginary frequency: $-429.9946 \mathrm{~cm}^{-1}$

TC[G] $(\omega B 97 X-D / c c-p V D Z$ at $298.15 \mathrm{~K})=0.303408$ au

TC[G] ( $\omega B 97 X-D / c c-p V D Z$ at $195.15 \mathrm{~K})=0.325413 \mathrm{au}$

$E$ (B2PLYP-D3/ aug-cc-pVTZ in gas phase) $=-872.009961$ au

$E(B 2 P L Y P-D 3 /$ aug-cc-pVTZ in THF) $=-872.031111$ au 


\section{SUPPORTING INFORMATION}

TS cis_3butoxy_3tbutyl

\begin{tabular}{|c|c|c|c|}
\hline 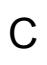 & -1.17961100 & -0.82758600 & 1.21674200 \\
\hline 1 & -0.63264300 & -1.30421300 & 2.04374600 \\
\hline $\mathrm{H}$ & -1.88551500 & -0.08598100 & 1.61003100 \\
\hline & -0.60705900 & -1.65250400 & -0.74257000 \\
\hline 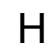 & -0.88947800 & -1.51843300 & -1.79376400 \\
\hline | & 0.13929400 & -2.45704200 & -0.65202200 \\
\hline & -0.19862500 & -0.39575800 & 0.09495100 \\
\hline & -1.71648300 & -1.91160300 & 0.28198800 \\
\hline & 2.14407000 & -0.30223800 & -0.35894300 \\
\hline & 3.45822300 & -0.69659000 & 0.28898600 \\
\hline & 1.94298800 & -0.94565200 & -1.23951300 \\
\hline & 2.20310100 & 0.74150900 & -0.72486200 \\
\hline & 4.65018500 & -0.54701800 & -0.65295300 \\
\hline & 3.60539300 & -0.07339900 & 1.18738500 \\
\hline & 3.38010900 & -1.73925600 & 0.64082400 \\
\hline & 4.48488600 & -1.16206100 & -1.55526700 \\
\hline & 4.71176500 & 0.49835000 & -1.00345200 \\
\hline U & -0.28100800 & 2.14681300 & 0.32065600 \\
\hline C & -0.64121500 & 3.46747600 & -0.35550300 \\
\hline & -0.86772500 & 2.04306700 & 1.25059200 \\
\hline П & 0.77707500 & 2.16235500 & 0.63297400 \\
\hline П & -0.05982200 & 3.56833300 & -1.28925700 \\
\hline & -1.70259900 & 3.44181700 & -0.65822200 \\
\hline o & -2.28892200 & -2.99099500 & 0.56975000 \\
\hline i & -4.05882800 & -2.90663900 & 0.24893600 \\
\hline - & -2.86156000 & -1.00247900 & -0.47860000 \\
\hline$\sqrt{1}$ & -4.38453500 & -0.22553700 & -0.55982500 \\
\hline & -5.26758300 & -1.59373300 & -0.14830300 \\
\hline $\mathrm{H}$ & -4.66421600 & 0.21165100 & -2.07199100 \\
\hline & -4.38099100 & 0.83461800 & 0.64201000 \\
\hline C & -0.52995000 & 0.93655900 & -0.57320300 \\
\hline & -1.58624400 & 0.91198800 & -0.88631100 \\
\hline$H$ & 0.05456400 & 1.02901200 & -1.50562800 \\
\hline & -0.39153700 & 4.67943000 & 0.53660400 \\
\hline & 0.66861600 & 4.74385800 & 0.83090200 \\
\hline & -0.65624900 & 5.61777300 & 0.02659900 \\
\hline & -0.98870700 & 4.61996100 & 1.46074100 \\
\hline 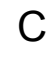 & 5.97155500 & -0.94406300 & -0.00142500 \\
\hline & 5.95042900 & -1.99498800 & 0.32879000 \\
\hline
\end{tabular}




\section{SUPPORTING INFORMATION}
$\mathrm{H}$
$6.81519500-0.82759200-0.69791400$
$\mathrm{H}$
$6.17834400-0.32272100 \quad 0.88461700$
$\mathrm{O}$
$1.11839100 \quad-0.44180000 \quad 0.60085400$

1 imaginary frequency: $-393.9762 \mathrm{~cm}^{-1}$

TC[G] $(\omega B 97 X-D / c c-p V D Z$ at $298.15 \mathrm{~K})=0.301665 \mathrm{au}$ TC[G] $(\omega B 97 X-D / c c-p V D Z$ at $195.15 \mathrm{~K})=0.324164 \mathrm{au}$

E (B2PLYP-D3/ aug-cc-pVTZ in gas phase $)=-872.013265$ au

$E(B 2 P L Y P-D 3 /$ aug-cc-pVTZ in THF) $=-872.034333 \mathrm{au}$

\section{TS' ${ }_{\text {trans_3butoxy_3tbutyl }}$}

C

$\begin{array}{lll}-1.20983600 & 0.34416500 & -1.10961300\end{array}$

$\mathrm{H}$

$\begin{array}{lll}-0.39744000 & 0.63176400 & -1.78509000\end{array}$

$\mathrm{H}$

$-2.08954500$

$0.08735000-1.71481000$

C

$-1.20983400$

0.34417100

1.10961400

$\mathrm{H}$

$-2.08954100$

0.08735800

1.71481400

$\mathrm{H}$

$-0.39743600$

$0.63177300 \quad 1.78508700$

C

$-0.83409600$

$-0.69451000$

0.00000300

C

1.55113300

$-0.16517600-0.00000100$

C

2.87250300

$-0.91145200-0.00000200$

$\mathrm{H}$

1.49290200

$0.49215300-0.88795600$

$\mathrm{H}$

1.49290500

0.49215600

0.88795200

C

4.07364900

$0.03111000-0.00000500$

$\mathrm{H}$

$\begin{array}{llll}2.90700700 & -1.57006600 & 0.88438800\end{array}$

$\mathrm{H}$

2.90700500

$-1.57006800-0.88439100$

$\mathrm{H}$

4.02001700

$0.69404200-0.88127400$

$\mathrm{H}$

4.02002000

$0.69404300 \quad 0.88126300$

$\mathrm{O}$

$\begin{array}{llll}0.51379200 & -1.11903400 & 0.00000200\end{array}$

C

5.40681400

$-0.71084100-0.00000600$

$\mathrm{H}$

5.50173000

$-1.35557700-0.88851300$

$\mathrm{H}$

$6.25627500-0.01168300-0.00000800$

$\mathrm{H}$

$5.50173300-1.35557500$

0.88850100

C

$-1.57791100$

1.33859500

$-0.00000200$

$\mathrm{H}$

$-0.15249500$

$2.14692900-0.00000600$

$\mathrm{O}$

$-2.53075100$

$2.15166200-0.00000300$

$\mathrm{Li}$

$-2.38559700$

$3.95699000-0.00000500$

Al

0.18885800

$3.79116500-0.00000800$

$\mathrm{H}$

$-0.78167500$

$4.25496900-1.25669800$

$\mathrm{H}$

1.73091800

$4.18935100-0.00001000$

$\mathrm{H}$

$-0.78167200$

$4.25497100 \quad 1.25668400$ 


\section{SUPPORTING INFORMATION}

$\begin{array}{lrrr}\text { C } & -1.66281900 & -2.01030200 & 0.00000700 \\ \text { C } & -1.30415900 & -2.81897900 & -1.25599100 \\ \text { C } & -1.30415600 & -2.81897300 & 1.25600800 \\ \text { C } & -3.17739800 & -1.75129600 & 0.00000800 \\ \text { H } & -1.56132200 & -2.26549800 & -2.17365600 \\ \text { H } & -0.23053200 & -3.04548700 & -1.28420500 \\ \text { H } & -1.86384900 & -3.76732600 & -1.27188800 \\ \text { H } & -1.56131800 & -2.26548600 & 2.17367000 \\ \text { H } & -1.86384600 & -3.76732000 & 1.27191100 \\ \text { H } & -0.23052900 & -3.04548000 & 1.28422100 \\ \text { H } & -3.70747100 & -2.71621200 & 0.00001100 \\ \text { H } & -3.51393600 & -1.19865000 & 0.88969700 \\ \text { H } & -3.51393800 & -1.19865500 & -0.88968400\end{array}$

1 imaginary frequency: $-488.7781 \mathrm{~cm}^{-1}$

TC[G] ( $\omega B 97 X-D / c c-p V D Z$ at $298.15 \mathrm{~K})=0.302061 \mathrm{au}$ TC[G] $(\omega B 97 X-D / c c-p V D Z$ at $195.15 \mathrm{~K})=0.324631 \mathrm{au}$ $\mathrm{E}$ (B2PLYP-D3/ aug-cc-pVTZ in gas phase $)=-872.013942$ au E (B2PLYP-D3/ aug-cc-pVTZ in THF) $=-872.033333$ au TS' ${ }_{\text {cis_3butoxy_3tbutyl }}$

$\begin{array}{llrr}\text { C } & -0.79164600 & -0.63781600 & -0.89099300 \\ H & -1.19304500 & -0.12197700 & -1.76967600 \\ \text { H } & -0.15018900 & -1.46247000 & -1.22683100 \\ \mathrm{C} & -1.23675800 & -0.25573600 & 1.24408400 \\ \mathrm{H} & -0.76940500 & -0.86275100 & 2.03107500 \\ \mathrm{H} & -1.91527800 & 0.47603300 & 1.69487300 \\ \mathrm{C} & -0.17548700 & 0.26894300 & 0.22736800 \\ \mathrm{C} & 2.02291400 & -0.72450500 & -0.21361900 \\ \mathrm{C} & 3.42614200 & -0.46991100 & 0.30721200 \\ \mathrm{H} & 1.84733500 & -1.81600600 & -0.28821500 \\ \mathrm{H} & 1.91862200 & -0.30674800 & -1.23049300 \\ \mathrm{C} & 4.50411700 & -1.12771900 & -0.55032800 \\ \mathrm{H} & 3.59392900 & 0.62011200 & 0.35603300 \\ \mathrm{H} & 3.48676200 & -0.84029500 & 1.34449400 \\ \mathrm{H} & 4.32141600 & -2.21569200 & -0.59812300 \\ \mathrm{H} & 4.42329800 & -0.75874300 & -1.58819100 \\ \mathrm{C} & -1.80945400 & -1.14572600 & 0.13717600 \\ \mathrm{H} & -3.03963400 & -0.14223200 & -0.31798200 \\ \mathrm{O} & -2.24221000 & -2.31468000 & 0.24866900 \\ \mathrm{Li} & -3.98660900 & -2.80503300 & 0.27964100\end{array}$




\section{SUPPORTING INFORMATION}

$\begin{array}{lrrr}\text { Al } & -4.69637600 & -0.40868900 & -0.35355100 \\ \text { H } & -4.72971200 & -1.75359100 & -1.31706700 \\ \text { H } & -5.60971200 & 0.84565000 & -0.71219600 \\ \text { H } & -4.87474600 & -1.14919300 & 1.11686300 \\ \text { C } & 5.91418700 & -0.87059200 & -0.02714200 \\ \text { H } & 6.03298800 & -1.25875200 & 0.99712400 \\ \text { H } & 6.67334200 & -1.35394000 & -0.65998500 \\ \text { H } & 6.13618100 & 0.20828200 & -0.00014200 \\ \text { O } & 1.10626100 & -0.13965700 & 0.68788400 \\ \mathrm{C} & -0.17120700 & 1.80556600 & -0.02214700 \\ \mathrm{C} & 0.25956400 & 2.50441800 & 1.27586100 \\ \mathrm{C} & 0.83028900 & 2.15272700 & -1.13377000 \\ \mathrm{C} & -1.55292000 & 2.33082400 & -0.43514500 \\ \mathrm{H} & -0.47004000 & 2.33690100 & 2.08384100 \\ \mathrm{H} & 1.23440200 & 2.12820400 & 1.61453100 \\ \mathrm{H} & 0.33623500 & 3.59152200 & 1.11737000 \\ \mathrm{H} & 0.60203200 & 1.61791600 & -2.07025200 \\ \mathrm{H} & 0.79200600 & 3.23125800 & -1.35294900 \\ \mathrm{H} & 1.86218400 & 1.91388400 & -0.83851300 \\ \mathrm{H} & -1.50270500 & 3.42239100 & -0.56975600 \\ \mathrm{H} & -1.90104400 & 1.89985200 & -1.38589900 \\ \mathrm{H} & -2.32336400 & 2.13029300 & 0.32355300\end{array}$

1 imaginary frequency: $-512.2647 \mathrm{~cm}^{-1}$

TC[G] $(\omega B 97 X-D / c c-p V D Z$ at $298.15 \mathrm{~K})=0.302729 \mathrm{au}$

TC[G] ( $\omega B$ 97X-D/cc-pVDZ at $195.15 \mathrm{~K})=0.325037 \mathrm{au}$

$E$ (B2PLYP-D3/ aug-cc-pVTZ in gas phase $)=-872.011448$ au

E (B2PLYP-D3/ aug-cc-pVTZ in THF) $=-872.031351 \mathrm{au}$

\section{TS $_{\text {trans_spirocyclopentane }}$}

$\begin{array}{llll}\mathrm{C} & -0.36153700 & 0.97191100 & -1.57288200 \\ \mathrm{C} & -1.68188100 & 0.29238300 & -1.13839200 \\ \mathrm{H} & -0.41526600 & 1.33466900 & -2.61378100 \\ \mathrm{H} & -2.47949500 & 0.41260400 & -1.89220500 \\ \mathrm{C} & -1.36550000 & -1.20958900 & -0.94045700 \\ \mathrm{C} & -1.31203100 & -1.67802900 & 0.54422300 \\ \mathrm{C} & -2.54626500 & -2.18429900 & -1.19231600 \\ \mathrm{H} & -1.22976200 & -0.90754700 & 1.31679000 \\ \mathrm{H} & -0.54150900 & -2.44809600 & 0.70440700 \\ \mathrm{H} & -3.41949000 & -1.82063400 & -1.75078900\end{array}$




\section{SUPPORTING INFORMATION}

$\mathrm{H}$

$\mathrm{O}$

$\mathrm{O}$

C

C

$\mathrm{H}$

$\mathrm{H}$

$\mathrm{H}$

C

$\mathrm{H}$

$\mathrm{H}$

$\mathrm{H}$

C

$\mathrm{H}$

C

$\mathrm{H}$

$\mathrm{H}$

$\mathrm{O}$

$\mathrm{Si}$

C

C

C

C

$\mathrm{H}$

$\mathrm{H}$

$\mathrm{H}$

$\mathrm{H}$

$\mathrm{H}$

$\mathrm{H}$

$\mathrm{H}$

$\mathrm{H}$

$\mathrm{H}$

C

$\mathrm{H}$

$\mathrm{O}$

C

$\mathrm{H}$

$\mathrm{H}$

$\mathrm{H}$

C
$-2.20917000 \quad-3.14142500-1.62424400$

$\begin{array}{lll}-2.03595600 & 0.97269200 & 0.04552700\end{array}$

$\begin{array}{lll}-0.17928300 & 2.04376100 & -0.68337500\end{array}$

$\begin{array}{lll}-1.38285400 & 2.23686000 & 0.05390000\end{array}$

$\begin{array}{llll}-2.25355200 & 3.28587400 & -0.62711300\end{array}$

$\begin{array}{llll}-3.19549400 & 3.41111600 & -0.07474400\end{array}$

$\begin{array}{lll}-1.72338700 & 4.24802900 & -0.66373700\end{array}$

$\begin{array}{lll}-2.49454000 & 2.98063200 & -1.65607700\end{array}$

$\begin{array}{lll}-1.03988500 & 2.57520700 & 1.48729500\end{array}$

$\begin{array}{lll}-0.46892400 & 3.51349000 & 1.52866400\end{array}$

$\begin{array}{lll}-1.96190100 & 2.68550800 & 2.07416700\end{array}$

$\begin{array}{lll}-0.43721700 & 1.76173100 & 1.91074100\end{array}$

$0.72357600 \quad-0.12688700-1.46010400$

$\begin{array}{llll}1.53711700 & 0.03199400 & -2.18887400\end{array}$

$\begin{array}{llll}-0.08794100 & -1.38463100 & -1.76897400\end{array}$

$\begin{array}{lll}-0.33948900 & -1.40786300 & -2.84382800\end{array}$

$0.46344700 \quad-2.30524300 \quad-1.53004900$

$1.21264400-0.21614100 \quad-0.14788100$

$\begin{array}{llll}2.68010700 & 0.47900700 & 0.37875100\end{array}$

$\begin{array}{lll}4.00000300 & -0.89689100 & 0.27323500\end{array}$

$3.62480300-2.02629700 \quad 1.24532600$

$4.04496100-1.45912700-1.15633600$

$5.37955900 \quad-0.33044800 \quad 0.64251300$

$3.63201700 \quad-1.68408200 \quad 2.29279600$

$2.62027300-2.42440400 \quad 1.02867100$

$4.34262200-2.86229600 \quad 1.16634200$

$4.29913000-0.68446400-1.89868000$

$4.80998200-2.25194300-1.23657900$

$3.07915400-1.90341500-1.44612000$

$6.14626400-1.12510600 \quad 0.60942500$

$\begin{array}{llll}5.69793900 & 0.46159200 & -0.05487700\end{array}$

$\begin{array}{lll}5.39349800 & 0.09263000 & 1.66053300\end{array}$

$\begin{array}{lll}-2.66312300 & -2.35979900 & 0.32568900\end{array}$

$\begin{array}{lll}-3.53905000 & -0.95129900 & 0.57916200\end{array}$

$\begin{array}{lll}-3.28326800 & -3.21720600 & 0.99155100\end{array}$

$\begin{array}{llll}3.13289400 & 1.94981200 & -0.69886000\end{array}$

$3.43276700 \quad 1.65380900-1.71622700$

$\begin{array}{llll}2.26433600 & 2.62195100 & -0.77261000\end{array}$

$\begin{array}{llll}3.97140200 & 2.50572500 & -0.24982200\end{array}$

$\begin{array}{lll}2.39572300 & 1.00015300 & 2.15631900\end{array}$ 


\section{SUPPORTING INFORMATION}

$\begin{array}{lrrr}\mathrm{H} & 1.82722700 & 0.22959300 & 2.69955200 \\ \mathrm{H} & 3.35092600 & 1.15715000 & 2.68201900 \\ \mathrm{H} & 1.82622900 & 1.94037200 & 2.19418800 \\ \mathrm{Li} & -4.77361100 & -2.25484300 & 1.50405800 \\ \mathrm{H} & -5.79530900 & -0.69661000 & 1.39994500 \\ \mathrm{Al} & -4.60018900 & 0.36836000 & 0.80729900 \\ \mathrm{H} & -4.17776000 & 1.33223900 & 2.01359500 \\ \mathrm{H} & -5.01957400 & 0.90727500 & -0.64228700\end{array}$

1 imaginary frequency: $-337.5842 \mathrm{~cm}^{-1}$

TC[G] $(\omega B 97 X-D / c c-p V D Z$ at $298.15 \mathrm{~K})=0.419260 \mathrm{au}$

TC[G] $(\omega B 97 X-D / c c-p V D Z$ at $195.15 \mathrm{~K})=0.446802 \mathrm{au}$

$E$ (B2PLYP-D3/ aug-cc-pVTZ in gas phase $)=-1506.690398$ au

E (B2PLYP-D3/ aug-cc-pVTZ in THF) $=-1506.714839$ au

TS $_{\text {cis_spirocyclopentane }}$

$\begin{array}{lrrr}\text { C } & -0.19525900 & 1.76757900 & -1.39212700 \\ \mathrm{C} & -1.63377300 & 1.73639100 & -0.82639000 \\ \mathrm{H} & -0.17403600 & 2.12536500 & -2.43580600 \\ \mathrm{H} & -2.35616500 & 2.20856800 & -1.51715300 \\ \mathrm{C} & -2.00185700 & 0.25183900 & -0.61692600 \\ \mathrm{C} & -2.02898200 & -0.25471400 & 0.85522000 \\ \mathrm{C} & -3.49620900 & -0.14711200 & -0.77022000 \\ \mathrm{H} & -2.15590600 & 0.57693200 & 1.56378300 \\ \mathrm{H} & -1.20396900 & -0.89470400 & 1.18511600 \\ \mathrm{H} & -4.17528900 & 0.71279100 & -0.64788100 \\ \mathrm{H} & -3.79297900 & -0.72397500 & -1.65546300 \\ \mathrm{O} & -1.55326400 & 2.47412000 & 0.37102800 \\ \mathrm{O} & 0.51672000 & 2.64413800 & -0.55786200 \\ \mathrm{C} & -0.39630900 & 3.29221300 & 0.32250000 \\ \mathrm{C} & -0.74582100 & 4.67019900 & -0.23194500 \\ \mathrm{H} & -1.47600300 & 5.17055000 & 0.41982500 \\ \mathrm{H} & 0.15806200 & 5.29155400 & -0.30354900 \\ \mathrm{H} & -1.18176200 & 4.57660800 & -1.23789300 \\ \mathrm{C} & 0.20886200 & 3.33513200 & 1.71019700 \\ \mathrm{H} & 1.14960000 & 3.90337300 & 1.69745900 \\ \mathrm{H} & -0.48625400 & 3.81446400 & 2.41357200 \\ \mathrm{H} & 0.40767400 & 2.30709000 & 2.03864900 \\ \mathrm{C} & 0.30327000 & 0.30324800 & -1.33364500 \\ \mathrm{H} & 1.03149300 & 0.09334700 & -2.13573900\end{array}$




\section{SUPPORTING INFORMATION}

C

$-0.99797900 \quad-0.47394000-1.52742900$

$\mathrm{H}$

$\begin{array}{lll}-1.32363300 & -0.39738300 & -2.57943700\end{array}$

$\mathrm{H}$

$-0.87493200-1.53831500-1.28722200$

O

$\begin{array}{llll}0.82996100 & 0.01030500 & -0.06545000\end{array}$

$\mathrm{Si}$

$\begin{array}{lll}2.49885400 & -0.09979500 & 0.27010200\end{array}$

C

$\begin{array}{llll}3.00762700 & -1.92390200 & 0.03886200\end{array}$

C

$\begin{array}{lll}2.28097500 & -2.78725500 & 1.08206900\end{array}$

C

$2.61185200-2.40113200-1.36755000$

C

$\begin{array}{lll}4.52788500 & -2.06226100 & 0.21587400\end{array}$

$\mathrm{H}$

$2.56513500-2.51257900$

2.11103400

$\mathrm{H}$

$1.18679200-2.69483700$

0.99216200

$\mathrm{H}$

$2.53207900-3.85391400$

0.94374900

$\mathrm{H}$

$3.08707600 \quad-1.79944200 \quad-2.16035300$

$\mathrm{H}$

$2.92477000 \quad-3.44922100-1.52031000$

$\mathrm{H}$

$\begin{array}{llll}1.52094100 & -2.36346900 & -1.51502400\end{array}$

$\mathrm{H}$

$\begin{array}{lll}4.83396100 & -3.11858900 & 0.11230900\end{array}$

$\mathrm{H}$

$\begin{array}{llll}5.08411800 & -1.48362000 & -0.53988000\end{array}$

$\mathrm{H}$

$\begin{array}{lll}4.86097900 & -1.72349300 & 1.21100700\end{array}$

C

$\begin{array}{llll}3.45907400 & 1.04087000 & -0.87500000\end{array}$

$\mathrm{H}$

$\begin{array}{llll}3.48982400 & 0.67226700 & -1.91218800\end{array}$

$\mathrm{H}$

$\begin{array}{llll}2.98237400 & 2.03341200 & -0.87223000\end{array}$

$\mathrm{H}$

$\begin{array}{llll}4.49837600 & 1.14776600 & -0.52557200\end{array}$

C

2.68626300

0.43193700

2.05861400

$\mathrm{H}$

3.66394300

0.12566800

2.46334400

$\mathrm{H}$

2.60790600

1.52588800

2.14489200

$\mathrm{H}$

$1.90021600-0.02175700$

2.68182900

C

$\begin{array}{lll}-3.39298000 & -0.87905700 & 0.57362600\end{array}$

$\mathrm{H}$

$\begin{array}{lll}-2.84744800 & -2.27145100 & -0.04889600\end{array}$

O

$\begin{array}{lll}-4.30659900 & -1.20051500 & 1.37549400\end{array}$

$\mathrm{Li}$

$-4.41839000 \quad-2.98834700 \quad 1.52654300$

Al

$-2.45000300-3.92185900-0.25586300$

$\mathrm{H}$

$-0.95375200-4.10296600$

0.28335800

$\mathrm{H}$

$\begin{array}{lll}-3.57322100 & -4.47656700 & 0.86259500\end{array}$

$\mathrm{H}$

$-2.86307500 \quad-4.33761600-1.74498100$

1 imaginary frequency: $-371.2919 \mathrm{~cm}^{-1}$

TC[G] $(\omega B 97 X-D / c c-p V D Z$ at $298.15 \mathrm{~K})=0.417502 \mathrm{au}$

TC[G] $(\omega B 97 X-D / c c-p V D Z$ at $195.15 \mathrm{~K})=0.445631 \mathrm{au}$

$\mathrm{E}$ (B2PLYP-D3/ aug-cc-pVTZ in gas phase) $=-1506.689512$ au

$E$ (B2PLYP-D3/ aug-cc-pVTZ in THF) $=-1506.713956$ au 
TS' ${ }_{\text {trans_spirocyclopentane }}$

C

$\begin{array}{lll}-0.27633000 & 1.10038500 & -1.64499800\end{array}$

C $\quad-1.63609900 \quad 0.46680200 \quad-1.26273200$

$\begin{array}{lllll}\mathrm{H} & -0.27292600 & 1.45791000 & -2.68899600\end{array}$

$\mathrm{H} \quad \begin{array}{lllll}\mathrm{H} & -2.41727900 & 0.66773100 & -2.01678600\end{array}$

C $\quad-1.39343200 \quad-1.05852500 \quad-1.13161700$

C $\quad \begin{array}{llll}-1.48186700 & -1.57521400 & 0.33081900\end{array}$

$\begin{array}{llll}\text { C } & -2.57783200 & -1.98955600 & -1.51389800\end{array}$

$\mathrm{H} \quad \begin{array}{llll}-1.40799300 & -0.84115700 & 1.13820500\end{array}$

$\begin{array}{llll}\mathrm{H} & -0.77625300 & -2.40114300 & 0.51445000\end{array}$

$\mathrm{H} \quad-3.38063300 \quad-1.60136700 \quad-2.15372000$

$\mathrm{H} \quad-2.21669100 \quad-2.94739100 \quad-1.92454900$

O $\quad-1.95833300 \quad 1.10529200-0.04568600$

$\begin{array}{lllll}0 & -0.09112100 & 2.17314200 & -0.75775300\end{array}$

$\begin{array}{llll}\text { C } & -1.28564600 & 2.35032500 & 0.00227200\end{array}$

C $\quad-2.14116500 \quad 3.44375400 \quad-0.62852700$

$\begin{array}{llll}\mathrm{H} & -3.08485200 & 3.54938000 & -0.07506800\end{array}$

$\begin{array}{lllll}\mathrm{H} & -1.60175400 & 4.40142600 & -0.61568900\end{array}$

$\mathrm{H} \quad \begin{array}{lllll}\mathrm{H} & -2.37561700 & 3.19378100 & -1.67400200\end{array}$

C $\quad-0.92413700 \quad 2.62815400 \quad 1.44515800$

$\begin{array}{llll}\mathrm{H} & -0.31495100 & 3.54031100 & 1.51543300\end{array}$

$\begin{array}{llll}\mathrm{H} & -1.83692600 & 2.76189400 & 2.04255600\end{array}$

$\begin{array}{llll}\mathrm{H} & -0.35612900 & 1.77588400 & 1.83840100\end{array}$

C $\quad 0.76348400 \quad-0.03356000 \quad-1.48422300$

$\begin{array}{llll}\mathrm{H} & 1.62736700 & 0.11383300 & -2.15523300\end{array}$

C $\quad-0.06460800 \quad-1.25642900-1.87054700$

$\mathrm{H} \quad-0.23961000-1.25387300 \quad-2.96079400$

$\mathrm{H} \quad 0.43786800 \quad-2.19953000-1.61173000$

O $\quad \begin{array}{llll}1.16260700 & -0.15733100 & -0.14301700\end{array}$

Si $\quad \begin{array}{llll}2.65718100 & 0.40026000 & 0.46027200\end{array}$

C $\quad 3.89587000 \quad-1.04721800 \quad 0.32011700$

$\begin{array}{llll}\text { C } & 3.41815600 & -2.20196000 & 1.21436400\end{array}$

C $\quad 3.96712300 \quad-1.53628800 \quad-1.13501000$

C $\quad 5.29053200 \quad-0.58433200 \quad 0.76907600$

$\mathrm{H} \quad 3.40560500 \quad-1.91721100 \quad 2.27884100$

$\begin{array}{llll}\mathrm{H} & 2.40063300 & -2.52545500 & 0.94133600\end{array}$

H $\quad 4.08788700 \quad-3.07479800 \quad 1.11405100$

$\mathrm{H} \quad 4.28669300 \quad-0.73859700 \quad-1.82588700$

H $\quad 4.69552300 \quad-2.36143800 \quad-1.22974700$ 


\section{SUPPORTING INFORMATION}

$\begin{array}{lrrr}\text { H } & 2.99359000 & -1.91712000 & -1.48289300 \\ \text { H } & 6.01093900 & -1.42038200 & 0.71894900 \\ \text { H } & 5.68026600 & 0.22339900 & 0.12815400 \\ \text { H } & 5.28979500 & -0.21757100 & 1.80876100 \\ \mathrm{C} & -2.84425400 & -2.17967600 & -0.01304800 \\ \mathrm{H} & -3.71316500 & -0.74336400 & 0.02414000 \\ \mathrm{O} & -3.51490400 & -3.03896700 & 0.59498000 \\ \mathrm{C} & 3.23418300 & 1.89868900 & -0.51546300 \\ \mathrm{H} & 3.57237100 & 1.63947400 & -1.53084100 \\ \mathrm{H} & 2.39921100 & 2.61136100 & -0.59930300 \\ \mathrm{H} & 4.07148800 & 2.39331300 & 0.00220200 \\ \mathrm{C} & 2.34825800 & 0.83633100 & 2.25729800 \\ \mathrm{H} & 1.86061700 & 1.81891500 & 2.33907200 \\ \mathrm{H} & 1.69516600 & 0.08637800 & 2.72969100 \\ \mathrm{H} & 3.29203300 & 0.87624600 & 2.82396600 \\ \mathrm{Li} & -4.49717700 & -2.41720900 & 2.02967500 \\ \mathrm{H} & -5.75223800 & -1.01812200 & 1.40999600 \\ \mathrm{Al} & -4.45683600 & 0.03724200 & 1.29538900 \\ \mathrm{H} & -3.57218700 & -0.58718500 & 2.54734400 \\ \mathrm{H} & -4.79738800 & 1.59112900 & 1.23436500\end{array}$

1 imaginary frequency: $-477.8730 \mathrm{~cm}^{-1}$

TC[G] ( $\omega B$ B97X-D/cc-pVDZ at $298.15 \mathrm{~K})=0.419775 \mathrm{au}$ TC[G] ( $\omega B$ B97X-D/cc-pVDZ at $195.15 \mathrm{~K})=0.447276 \mathrm{au}$ $E$ (B2PLYP-D3/ aug-cc-pVTZ in gas phase $)=-1506.688920 \mathrm{au}$ $E$ (B2PLYP-D3/ aug-cc-pVTZ in THF) $=-1506.711859$ au

\section{TS' ${ }_{\text {cis_spirocyclopentane }}$}

$\begin{array}{lrrr}\text { C } & 0.00971100 & 1.85955600 & -1.35181700 \\ \text { C } & -1.45236100 & 1.88809100 & -0.84764100 \\ \text { H } & 0.10073600 & 2.28344100 & -2.36671500 \\ \text { H } & -2.11253600 & 2.44509400 & -1.53735700 \\ \text { C } & -1.91960800 & 0.42079000 & -0.74287900 \\ \text { C } & -2.03149100 & -0.17010100 & 0.69296700 \\ \text { C } & -3.42593000 & 0.11679400 & -0.97230300 \\ \text { H } & -2.16046600 & 0.62826500 & 1.43825200 \\ \text { H } & -1.24691700 & -0.85643800 & 1.02674600 \\ \text { H } & -4.06094300 & 1.00142800 & -0.80144700 \\ \text { H } & -3.72342900 & -0.36904800 & -1.91043100 \\ \text { O } & -1.37882400 & 2.55010100 & 0.39427500\end{array}$




\section{SUPPORTING INFORMATION}

$\mathrm{O}$

C

C

$\mathrm{H}$

$\mathrm{H}$

$\mathrm{H}$

C

$\mathrm{H}$

$\mathrm{H}$

$\mathrm{H}$

C

$\mathrm{H}$

C

$\mathrm{H}$

$\mathrm{H}$

O

$\mathrm{Si}$

C

C

C

C

$\mathrm{H}$

$\mathrm{H}$

$\mathrm{H}$

$\mathrm{H}$

$\mathrm{H}$

$\mathrm{H}$

$\mathrm{H}$

$\mathrm{H}$

$\mathrm{H}$

C

$\mathrm{H}$

$\mathrm{H}$

$\mathrm{H}$

C

$\mathrm{H}$

$\mathrm{H}$

$\mathrm{H}$

C

$\mathrm{H}$
0.73978400

$-0.16963500$

2.62797700

3.28724600

$-0.43101900$

$-0.39959100$

4.71535300

0.44494800

$-1.12579400$

5.22722100

$-0.04155100$

0.54577600

5.27617300

0.60572200

$-0.79205600$

$4.70875300-1.06951400$

0.37086800

3.21081100

1.34705600

3.71222900

1.85784300

$-0.32327300$

3.69735200

1.91948400

0.48433200

2.15484700

2.55721800

0.41046600

0.36471800

2.13426300

1.15655100

0.16040500

$-1.37473500$

$-0.92927800$

$-1.20773600$

$-0.13558400$

$-0.89234800$

$-1.39368400$

0.86547100

$-0.04537200$

2.50239800

$-0.29457600$

0.28934400

2.87900900

$-2.13763300$

$-0.03461300$

1.98843200

$-2.99739000$

0.87655700

$2.57247000 \quad-2.48109000-1.50105300$

$\begin{array}{lll}4.35906600 & -2.42857600 & 0.25800400\end{array}$

$2.20415800-2.82385700$

$0.91975700-2.78574300$

1.94328700

$2.15072000-4.07150100$

0.70964700

$3.17236500-1.87845100-2.20305200$

$2.80018800 \quad-3.54272200-1.70365500$

$1.50842700-2.32521900-1.74013800$

$\begin{array}{llll}4.58347000 & -3.49743000 & 0.09211900\end{array}$

$5.02889600-1.84748400-0.39711900$

$\begin{array}{llll}4.62698000 & -2.19818700 & 1.30233100\end{array}$

$\begin{array}{llll}3.59706100 & 0.83591100 & -0.73843600\end{array}$

$\begin{array}{llll}3.63981500 & 0.53674900 & -1.79724000\end{array}$

$\begin{array}{llll}3.19909300 & 1.86118600 & -0.68324600\end{array}$

$\begin{array}{llll}4.62666300 & 0.83567900 & -0.34658600\end{array}$

$\begin{array}{lll}2.63971000 & 0.11189900 & 2.11454800\end{array}$

$\begin{array}{lll}3.57679800 & -0.27967300 & 2.54104000\end{array}$

$\begin{array}{lll}2.62366000 & 1.20144300 & 2.26636700\end{array}$

$\begin{array}{lll}1.79839800 & -0.32687700 & 2.67293200\end{array}$

$\begin{array}{lll}-3.40848500 & -0.72151800 & 0.31635800\end{array}$

$\begin{array}{llll}-2.86563500 & -2.03382700 & -0.41501800\end{array}$ 


\section{SUPPORTING INFORMATION}

$\mathrm{O}$

$\mathrm{Li}$

Al

$\mathrm{H}$

$\mathrm{H}$

$\mathrm{H}$ $\begin{array}{lll}-4.37094300 & -1.02027000 & 1.06512000\end{array}$

$\begin{array}{lll}-4.67718100 & -2.68205800 & 1.70657800\end{array}$

$\begin{array}{lll}-2.96673200 & -3.65574800 & 0.03054800\end{array}$

$\begin{array}{lll}-2.76140100 & -3.49342000 & 1.66116100\end{array}$

$-4.61052000 \quad-3.81925700-0.01463700$

$\begin{array}{lll}-2.03170600 & -4.65297000 & -0.78687400\end{array}$

1 imaginary frequency: $-497.4289 \mathrm{~cm}^{-1}$

TC[G] $(\omega B 97 X-D / c c-p V D Z$ at $298.15 \mathrm{~K})=0.417940 \mathrm{au}$ TC[G] ( $\omega B$ 97X-D/cc-pVDZ at $195.15 \mathrm{~K})=0.446067 \mathrm{au}$ $E$ (B2PLYP-D3/ aug-cc-pVTZ in gas phase) $=-1506.689641 \mathrm{au}$ $E$ (B2PLYP-D3/ aug-cc-pVTZ in THF) $=-1506.712563$ au

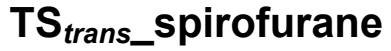

$\begin{array}{lrrr}\text { C } & 1.37735000 & -0.32672600 & -1.54145800 \\ \text { C } & 2.08825900 & -1.46273100 & -0.79628800 \\ \text { C } & 0.85240200 & -1.71908300 & 0.07259400 \\ \text { H } & 1.89748200 & 0.62510100 & -1.68529200 \\ \text { H } & 1.02880700 & -0.70956400 & -2.51492300 \\ \text { H } & 2.84668600 & -0.43995300 & 0.28654900 \\ \text { H } & 0.97326900 & -1.80651700 & 1.15795700 \\ \text { H } & 0.30279600 & -2.59163400 & -0.31259500 \\ \mathrm{C} & 0.26872500 & -0.39109200 & -0.44863600 \\ \mathrm{C} & -1.68937500 & 1.04313700 & -0.47694000 \\ \mathrm{C} & -0.45244800 & 1.69593200 & 0.13804500 \\ \mathrm{H} & -2.09402800 & 1.65373700 & -1.29750800 \\ \mathrm{H} & -0.68156700 & 2.27477000 & 1.04778600 \\ \mathrm{O} & 0.37250500 & 0.62768700 & 0.53774000 \\ \mathrm{C} & -1.19496300 & -0.35665200 & -0.90084300 \\ \mathrm{H} & -1.30560100 & -0.56264600 & -1.97713500 \\ \mathrm{C} & -3.06528800 & -0.55531800 & 0.42673500 \\ \mathrm{O} & -1.96406000 & -1.25662400 & -0.13435400 \\ \mathrm{O} & 0.13373500 & 2.49058200 & -0.84700800 \\ \mathrm{C} & 1.26892800 & 3.19983100 & -0.38841300 \\ \mathrm{H} & 2.08031500 & 2.52067400 & -0.07847200 \\ \mathrm{H} & 1.61127700 & 3.82696600 & -1.22103700 \\ \mathrm{H} & 1.00966900 & 3.84736100 & 0.46928800 \\ \mathrm{O} & -2.64563000 & 0.79485000 & 0.52608400 \\ \mathrm{C} & -4.27934800 & -0.66719700 & -0.49074300 \\ \mathrm{H} & -4.57729600 & -1.71928800 & -0.60173200\end{array}$




\section{SUPPORTING INFORMATION}

$\begin{array}{llll}\mathrm{H} & -5.12250800 & -0.09595000 & -0.07754600 \\ \mathrm{H} & -4.04481000 & -0.26612400 & -1.48838500 \\ \mathrm{C} & -3.31038300 & -1.08558000 & 1.82386900 \\ \mathrm{H} & -4.12758400 & -0.52907300 & 2.30297000 \\ \mathrm{H} & -3.57965900 & -2.14999600 & 1.78375600 \\ \mathrm{H} & -2.39394900 & -0.96425100 & 2.41593500 \\ \mathrm{O} & 2.98967100 & -2.24254000 & -1.17485800 \\ \mathrm{Li} & 4.45350800 & -1.75086200 & -0.19555600 \\ \mathrm{H} & 3.99555500 & 1.72576200 & 1.37017800 \\ \mathrm{Al} & 3.72542600 & 0.16860300 & 1.61957200 \\ \mathrm{H} & 3.02804600 & -0.38717400 & 2.94585800 \\ \mathrm{H} & 5.08910900 & -0.75022100 & 1.23821700\end{array}$

1 imaginary frequency: $-355.2099 \mathrm{~cm}^{-1}$

TC[G] $(\omega B 97 X-D / c c-p V D Z$ at $298.15 \mathrm{~K})=0.246765 \mathrm{au}$

TC[G] $(\omega B 97 X-D / c c-p V D Z$ at $195.15 \mathrm{~K})=0.268383$ au

$E$ (B2PLYP-D3/ aug-cc-pVTZ in gas phase $)=-1055.794489$ au

$E$ (B2PLYP-D3/ aug-cc-pVTZ in THF) $=-1055.818641$ au

\section{TS $_{\text {cis_spirofurane }}$}

$\begin{array}{lrrr}\text { O } & -0.80714000 & 1.48483100 & 0.95633400 \\ \mathrm{C} & -1.83648800 & 1.48418100 & -0.00102800 \\ \mathrm{C} & -1.76650200 & 0.11904500 & -0.67836800 \\ \mathrm{C} & -0.30171000 & -0.31904800 & -0.46614400 \\ \mathrm{H} & -2.78103200 & 1.65125600 & 0.54253700 \\ \mathrm{H} & -2.07457000 & 0.18313000 & -1.73243900 \\ \mathrm{H} & 0.25582500 & -0.46964400 & -1.40217600 \\ \mathrm{C} & 0.30228300 & 0.80355000 & 0.39594600 \\ \mathrm{C} & 1.35421200 & 0.39365800 & 1.44384200 \\ \mathrm{C} & 1.36287400 & 1.67594800 & -0.33113000 \\ \mathrm{C} & 2.42586000 & 0.96367400 & 0.51171800 \\ \mathrm{H} & 1.39673700 & -0.65863400 & 1.74428200 \\ \mathrm{H} & 1.27580800 & 1.04322700 & 2.32973500 \\ \mathrm{H} & 1.42638300 & 1.64977200 & -1.42588800 \\ \mathrm{H} & 1.30398700 & 2.72013100 & 0.01262900 \\ \mathrm{H} & 2.50634600 & -0.37613500 & -0.45696100 \\ \mathrm{O} & -0.38869900 & -1.52212100 & 0.26250500 \\ \mathrm{O} & -1.63433400 & 2.44566800 & -0.99593500 \\ \mathrm{C} & -1.71287700 & 3.76954000 & -0.51375500 \\ \mathrm{H} & -0.95510900 & 3.96273500 & 0.26358100\end{array}$




\section{SUPPORTING INFORMATION}

$\begin{array}{llll}\mathrm{H} & -1.53880900 & 4.43503000 & -1.36838700 \\ \mathrm{H} & -2.71256800 & 3.97927100 & -0.08970500 \\ \mathrm{O} & 3.61687600 & 1.28084300 & 0.73858800 \\ \mathrm{O} & -2.52354700 & -0.82076200 & 0.04593500 \\ \mathrm{C} & -1.73140800 & -1.98564100 & 0.21830900 \\ \mathrm{C} & -1.91675700 & -2.93958000 & -0.95725000 \\ \mathrm{H} & -1.27198600 & -3.82088900 & -0.83451600 \\ \mathrm{H} & -2.96492400 & -3.26407500 & -1.02224000 \\ \mathrm{H} & -1.64473000 & -2.44516100 & -1.90179300 \\ \mathrm{C} & -2.07115600 & -2.60217600 & 1.55851200 \\ \mathrm{H} & -3.11816800 & -2.93514100 & 1.56886900 \\ \mathrm{H} & -1.41986600 & -3.46536200 & 1.75199900 \\ \mathrm{H} & -1.92226700 & -1.85067000 & 2.34481300 \\ \mathrm{Li} & 4.71616600 & 0.12238300 & -0.11605500 \\ \mathrm{H} & 4.64859500 & -1.47069900 & -1.03046100 \\ \mathrm{Al} & 3.03328300 & -1.93895700 & -0.92795600 \\ \mathrm{H} & 2.41275900 & -2.26107500 & -2.36555800 \\ \mathrm{H} & 2.84117800 & -2.89435800 & 0.33995800\end{array}$

1 imaginary frequency: $-381.6546 \mathrm{~cm}^{-1}$

TC[G] $(\omega B 97 X-D / c c-p V D Z$ at $298.15 \mathrm{~K})=0.246556 \mathrm{au}$ TC[G] $(\omega B 97 X-D / c c-p V D Z$ at $195.15 \mathrm{~K})=0.268331 \mathrm{au}$ $E$ (B2PLYP-D3/ aug-cc-pVTZ in gas phase) $=-1055.795917$ au $E$ (B2PLYP-D3/ aug-cc-pVTZ in THF) $=-1055.819910 \mathrm{au}$

\section{TS' trans_spirofurane}

C

$1.40755300-0.25208200-1.25634600$

C $\quad 2.11002600-1.35534000 \quad-0.45223900$

$\begin{array}{llll}\text { C } & 0.83024300 & -1.64127000 & 0.34659000\end{array}$

H $\quad \begin{array}{llll}1.89814700 & 0.71842700 & -1.37277000\end{array}$

$\mathrm{H} \quad 1.14703100-0.65302300 \quad-2.24978900$

$\mathrm{H} \quad 2.70532400 \quad-0.29999000 \quad 0.69630100$

H $\quad 0.88199600 \quad-1.68734300 \quad 1.43916000$

$\mathrm{H} \quad 0.34640300 \quad-2.55067000 \quad-0.04058200$

C $\quad 0.22303900 \quad-0.35632700 \quad-0.24971800$

C $\quad-1.76929600 \quad 1.00086900 \quad-0.51890200$

$\begin{array}{llll}\text { C } & -0.63133700 & 1.70719500 & 0.21913000\end{array}$

$\mathrm{H} \quad-2.08072500 \quad 1.57132200 \quad-1.40621300$

$\begin{array}{llll}\mathrm{H} & -0.98445600 & 2.28749800 & 1.08779300\end{array}$

$\begin{array}{lllll}\mathrm{O} & 0.18621100 & 0.68128800 & 0.72207500\end{array}$ 


\section{SUPPORTING INFORMATION}

$\begin{array}{llrr}\text { C } & -1.19548000 & -0.40032600 & -0.82363700 \\ \text { H } & -1.20891500 & -0.66675300 & -1.89243700 \\ \text { C } & -3.18766700 & -0.60686100 & 0.30436600 \\ \text { O } & -1.99876400 & -1.28375000 & -0.07216100 \\ \text { O } & 0.03216300 & 2.51865900 & -0.70466600 \\ \text { C } & 1.10631800 & 3.23800900 & -0.13425800 \\ \text { H } & 1.87820000 & 2.56143400 & 0.27029000 \\ \text { H } & 1.53930100 & 3.85533300 & -0.93107200 \\ \text { H } & 0.75436200 & 3.89451400 & 0.68294600 \\ \text { O } & -2.84085600 & 0.76717200 & 0.36516800 \\ \text { C } & -4.27617800 & -0.83027400 & -0.74172200 \\ \text { H } & -4.51692300 & -1.89994100 & -0.81905100 \\ \text { H } & -5.18500500 & -0.27532000 & -0.46957500 \\ \text { H } & -3.93848000 & -0.47814700 & -1.72819100 \\ \text { C } & -3.58037300 & -1.06496300 & 1.69343800 \\ \text { H } & -4.46884500 & -0.51728400 & 2.03658100 \\ \text { H } & -3.80403400 & -2.14064800 & 1.68935500 \\ \text { H } & -2.74647500 & -0.87004400 & 2.38024300 \\ \text { O } & 3.00756400 & -2.14436600 & -0.81106000 \\ \text { Li } & 4.78892300 & -1.84957900 & -0.56641600 \\ \text { H } & 4.89486700 & -1.37159200 & 1.40831100 \\ \mathrm{Al} & 4.29339800 & 0.12027200 & 1.01267500 \\ \mathrm{H} & 4.86067200 & 0.20316700 & -0.54401300 \\ \mathrm{H} & 4.53498200 & 1.36047200 & 1.98211700\end{array}$

1 imaginary frequency: $-493.5976 \mathrm{~cm}^{-1}$

TC[G] ( $\omega B 97 X-D / c c-p V D Z$ at $298.15 \mathrm{~K})=0.248161 \mathrm{au}$ TC[G] $(\omega B 97 X-D / c c-p V D Z$ at $195.15 \mathrm{~K})=0.269483 \mathrm{au}$ $E$ (B2PLYP-D3/ aug-cc-pVTZ in gas phase $)=-1055.795123$ au $E$ (B2PLYP-D3/ aug-cc-pVTZ in THF) $=-1055.818458$ au

TS' ${ }_{\text {cis_s }}$ spirofurane

$\begin{array}{lrrr}\text { C } & -1.84013600 & 0.19397800 & -0.69956700 \\ \mathrm{C} & -1.81258100 & 1.53353900 & 0.03214500 \\ \mathrm{H} & -2.13995300 & 0.32203000 & -1.75011900 \\ \mathrm{H} & -2.73880400 & 1.73634800 & 0.59490000 \\ \mathrm{O} & -0.77482500 & 1.42860500 & 0.97385200 \\ \mathrm{C} & -0.41190600 & -0.35928800 & -0.50294900 \\ \mathrm{H} & 0.12917700 & -0.54783100 & -1.44165800 \\ \mathrm{C} & -1.95933600 & -1.92608700 & 0.16006600\end{array}$




\section{SUPPORTING INFORMATION}

$\mathrm{O}$

$\mathrm{O}$

C

$\mathrm{H}$

$\mathrm{H}$

$\mathrm{H}$

$\mathrm{O}$

C

$\mathrm{H}$

$\mathrm{H}$

$\mathrm{H}$

C

$\mathrm{H}$

$\mathrm{H}$

$\mathrm{H}$

C

C

C

C

$\mathrm{H}$

$\mathrm{H}$

$\mathrm{H}$

$\mathrm{H}$

$\mathrm{H}$

O

$\mathrm{Li}$

$\mathrm{H}$

Al

$\mathrm{H}$

$\mathrm{H}$ $\begin{array}{lll}-0.59059800 & -1.55603800 & 0.22185000\end{array}$

$\begin{array}{lll}-1.56363200 & 2.52126900 & -0.92593500\end{array}$

$\begin{array}{lll}-1.54981700 & 3.82566100 & -0.38822100\end{array}$

$\begin{array}{llll}-0.77070900 & 3.93682800 & 0.38403100\end{array}$

$\begin{array}{lll}-1.34445000 & 4.51432000 & -1.21718800\end{array}$

$\begin{array}{llll}-2.52814100 & 4.08078300 & 0.06037400\end{array}$

$\begin{array}{lll}-2.67185300 & -0.71154000 & -0.01336200\end{array}$

$-2.19903600 \quad-2.85750700-1.02441500$

$-1.61283700 \quad-3.77966000-0.90717300$

$-3.26537500 \quad-3.11387900-1.09766400$

$\begin{array}{lll}-1.89277400 & -2.37201300 & -1.96318400\end{array}$

$-2.35522400 \quad-2.52723300 \quad 1.49236800$

$-3.42418500 \quad-2.78123500 \quad 1.49188100$

$\begin{array}{lll}-1.77239100 & -3.43850200 & 1.68444400\end{array}$

$\begin{array}{lll}-2.15703600 & -1.79471700 & 2.28567600\end{array}$

$\begin{array}{lll}0.28190200 & 0.70414500 & 0.36418200\end{array}$

$\begin{array}{lll}1.33629200 & 0.20091300 & 1.36537200\end{array}$

$1.36742800 \quad 1.54423000-0.36535200$

$\begin{array}{lll}2.41730300 & 0.75146600 & 0.42523200\end{array}$

$\begin{array}{lll}1.32592800 & -0.86124800 & 1.63059700\end{array}$

$\begin{array}{lll}1.31661100 & 0.82038600 & 2.27555800\end{array}$

$\begin{array}{lll}1.40161700 & 1.55045500 & -1.46136200\end{array}$

$\begin{array}{lll}1.36634000 & 2.57852900 & 0.01038300\end{array}$

$2.36122100-0.51599800-0.59796600$

$3.60927600 \quad 1.04047100 \quad 0.67184600$

$\begin{array}{lll}4.99089500 & -0.12039200 & 0.46381000\end{array}$

$\begin{array}{lll}4.71907200 & -0.78543600 & -1.44736300\end{array}$

$\begin{array}{llll}3.48670400 & -1.73413800 & -0.87980700\end{array}$

$\begin{array}{lll}2.98529000 & -2.97875200 & -1.73479300\end{array}$

$\begin{array}{lll}4.02528300 & -1.95047700 & 0.66750800\end{array}$

1 imaginary frequency: $-493.4544 \mathrm{~cm}^{-1}$

TC[G] $(\omega B 97 X-D / c c-p V D Z$ at $298.15 \mathrm{~K})=0.248751 \mathrm{au}$

TC[G] ( $\omega B 97 X-D / c c-p V D Z$ at $195.15 \mathrm{~K})=0.269927 \mathrm{au}$

$E$ (B2PLYP-D3/ aug-cc-pVTZ in gas phase) $=-1055.797255$ au

$E$ (B2PLYP-D3/ aug-cc-pVTZ in THF) $=-1055.819665$ au 


\section{Screening enzymatic reductions}

Detailed protocols for the screening of the different ketoreductases can be found online (https://www.codexis-estore.com/protocols) and are described in below tables S4 and S5.
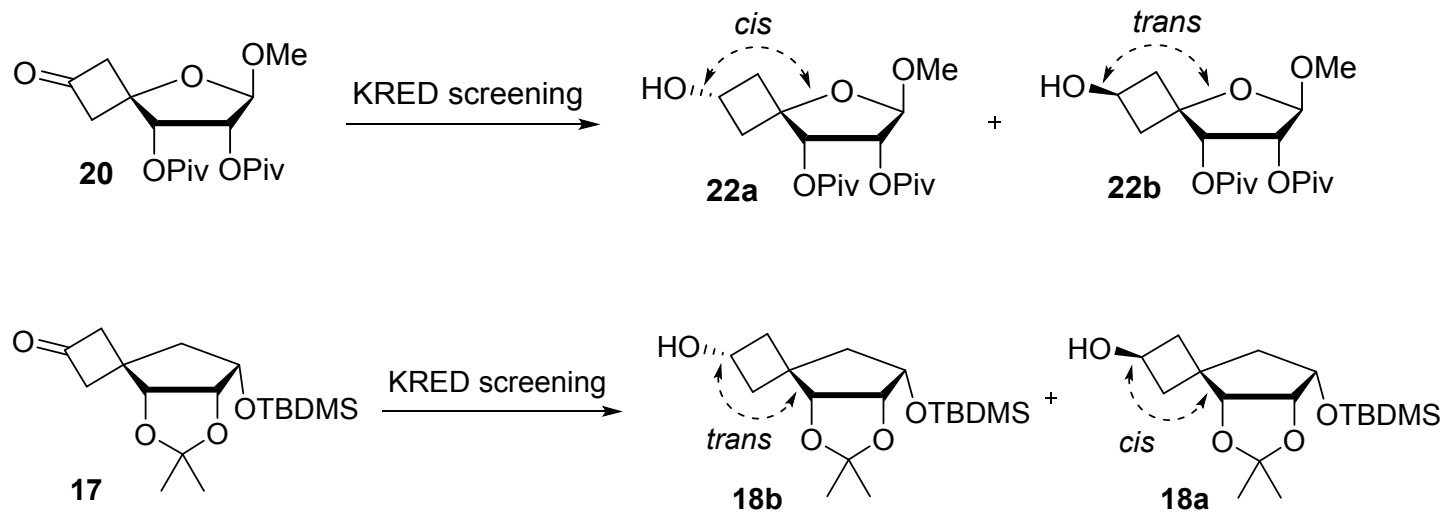

\begin{tabular}{|c|c|c|c|}
\hline Entry & $\begin{array}{l}\text { KRED } \\
\text { reference }\end{array}$ & $\begin{array}{c}\text { Conversion } \\
1 \%\end{array}$ & $\begin{array}{c}\text { Ratio } \\
18 b: 18 a\end{array}$ \\
\hline 1 & A04 & 45 & $97: 3$ \\
\hline 2 & A12 & 95 & $97: 3$ \\
\hline 3 & B02 & 15 & $85: 15$ \\
\hline 4 & B05 & 100 & 5:95 \\
\hline 5 & B10 & 0 & n.a. \\
\hline 6 & B12 & 0 & n.a. \\
\hline 7 & $\mathrm{C} 01$ & 95 & $12: 88$ \\
\hline 8 & H08 & 30 & n.a. \\
\hline 9 & B02 & 80 & $\geq 99 \% 18 a$ \\
\hline 10 & $\mathrm{CO2}$ & 0 & n.a. \\
\hline 11 & C11 & 20 & $\geq 99 \% 18 a$ \\
\hline 12 & D03 & 100 & $\geq 99 \% 18 a$ \\
\hline 13 & D11 & 55 & $\geq 99 \% 18 a$ \\
\hline 14 & D12 & 80 & $29: 71$ \\
\hline 15 & G03 & 0 & n.a. \\
\hline 16 & $\mathrm{H} 07$ & 77 & $\geq 99 \% 18 b$ \\
\hline 17 & B03 & 0 & n.a. \\
\hline 18 & G09 & 0 & n.a. \\
\hline 19 & H12 & 100 & $66: 33$ \\
\hline
\end{tabular}

\begin{tabular}{|c|c|c|c|}
\hline Entry & $\begin{array}{l}\text { KRED } \\
\text { reference }\end{array}$ & $\begin{array}{c}\text { Conversion } \\
1 \%\end{array}$ & $\begin{array}{c}\text { Ratio } \\
\text { 22a:22b }\end{array}$ \\
\hline 1 & A04 & 0 & n.a. \\
\hline 2 & A12 & 12 & $90: 10$ \\
\hline 3 & B02 & 0 & n.a. \\
\hline 4 & B05 & 59 & 5:95 \\
\hline 5 & B10 & 0 & n.a. \\
\hline 6 & B12 & 0 & n.a. \\
\hline 7 & $\mathrm{C} 01$ & 65 & $2: 98$ \\
\hline 8 & H08 & 0 & n.a. \\
\hline 9 & B02 & 93 & $\geq 99 \% \mathbf{2 2 b}$ \\
\hline 10 & $\mathrm{CO2}$ & 73 & $\geq 99 \% \mathbf{2 2 b}$ \\
\hline 11 & C11 & 7 & $\geq 99 \%$ 22b \\
\hline 12 & D03 & 63 & $87: 13$ \\
\hline 13 & D11 & 34 & $\geq 99 \% \mathbf{2 2 b}$ \\
\hline 14 & D12 & 12 & $54: 46$ \\
\hline 15 & G03 & 22 & $\geq 99 \% \mathbf{2 2 b}$ \\
\hline 16 & H07 & 4 & $\geq 99 \% \mathbf{2 2 b}$ \\
\hline 17 & B03 & 1 & n.a. \\
\hline 18 & G09 & 1 & n.a. \\
\hline 19 & H12 & 5 & $\geq 99 \% 22 b$ \\
\hline
\end{tabular}

Table S4. Screening KRED enzymes on cyclobutanone 17

Enzymes from entries 12 and 16 were selected for scale-up

Table S5. Screening KRED enzymes on cyclobutanone 20 Enzyme from entry 9 were selected for scale-up 


\section{SUPPORTING INFORMATION}

An initial screening was performed by weighing $10 \mathrm{mg}$ of each enzyme in a separate falcon tube. To these tubes was added $0.9 \mathrm{ml}$ of the freshly prepared KRED recycle mix $\mathrm{P}$ solution $(600 \mathrm{mg}$ KRED recycle mix $\mathrm{P}$ in $33 \mathrm{ml}$ deionized water) and gently mixed to avoid foam formation. Then, $1 \mathrm{mmol}$ of ketone substrates $\mathbf{2 0}$ and $\mathbf{1 7}$ was dissolved in $4 \mathrm{ml} \mathrm{iPrOH}$, and from this solution, $0.1 \mathrm{ml}$ was added to each falcon tube containing the enzyme/buffer solution. After shaking these tubes for 24 hours shaking at $33^{\circ} \mathrm{C}$, EtOAc $(2 \mathrm{ml})$ was added and the mixture was shaken, then centrifuged. The organic layer was collected, the solvent was removed in vacuo and the samples were analysed via ${ }^{1} \mathrm{H}$ NMR to determine the conversion from substrates (20-17) and the respective ratio of 22a22b and 18a-18b, respectively. For scale up of the enzymatic reductions, enzymes that showed high selectivity and turnover during the screening were selected. More detailed information, including the protocols for aforementioned screening reactions can be found on the company's website: https://www.codexis.com/products/screening-kits/ 


\section{SUPPORTING INFORMATION}

\section{In vitro inhibition studies}

Reagents. PRMT5:MEP50 protein was purchased from Charles River Laboratories (Wilmington, Massachusetts). The protein complex was produced in Sf9 insect cells infected simultaneously with two baculoviruses. One virus expresses full length human PRMT5 with Flag-tag at N-terminus, the second virus expresses full length MEP50 with His6-TEV cleavage sequence at N-terminus. The protein was affinity purified using anti-Flag beads eluted with 3xFlag peptide, followed by His-select eluted with $0.5 \mathrm{M}$ imidazole. Eluted protein was then dialyzed against TBS buffer, $\mathrm{pH} 8.0$ containing 20\% glycerol and 3 mM DTT. Full length recombinant human histone H2A (Genebank Accession\# NM_021052, Cat\# HMT-11-146) expressed in E. coli was purchased from Reaction Biology Corporation (Malvern, Pennsylvania). SAM (Cat\# 13956) and SAH (Cat\# 13603) were purchased from Cayman Chemical Company (Ann Arbor, Michigan). Reagents used for making reaction buffer and stopping reaction were purchased. Trizma base (Cat\# T1503), sodium chloride solution 5 M (Cat\# 59222C), magnesium chloride hexahydrate (Cat \# M0250) were purchased from Sigma-Aldrich Corporation (Ronkonkoma, NY). DTT solution 1 M (Cat\# P2325) was purchased from ThermoFisher Scientific (Waltham, MA). Formic acid (Cat\# 33015) was purchased from Riedel deHaen (Mexico City, Mexico).

High throughput mass spectrometry assay. Enzyme activity was measured by following the production of $\mathrm{SAH}$ generated by transferring a methyl group of SAM to histone H2A by PRMT5:MEP50. Assay was performed in a buffer of $20 \mathrm{mM}$ Tris- $\mathrm{HCl}, \mathrm{pH} 8.5,50 \mathrm{mM} \mathrm{NaCl}, 5$ $\mathrm{mM} \mathrm{MgCl} 2$ and $1 \mathrm{mM}$ DTT. Reaction was stopped by $1 \%$ formic acid (final concentration). Reaction substrate SAM and product SAH were detected using a RapidFire 300 high-throughput solid-phase extraction chromatography system coupled to a Sciex 4000 QTrap triple quadrupole mass spectrometry (Agilent Technologies, Santa Clara, CA). Reaction mixtures were injected onto an Agilent Graphite Type D cartridge in $0.1 \%$ trifluoacetic acid, and eluted with $80 \%$ acetonitrile and $0.1 \%$ trifluoacetic acid. The multiple reaction monitoring (MRM) method was used for detecting SAM and SAH at Q1/Q3 of 399.2/250.1 and 385.2/136.1, respectively.

Inhibition studies. Compound $I C_{50}$ values were determined using a 11-point dosing series made for each compound by 2-fold serially diluted in DMSO, with point 12 being a DMSO control for an uninhibited enzymatic reaction. Compounds were first spotted to 384-well assay plates, and followed by addition of $30 \mu \mathrm{l}$ of a mixture of $2 \mu \mathrm{M}$ SAM and $0.6 \mu \mathrm{M} \mathrm{H} 2 \mathrm{~A}$. The enzymatic 


\section{SUPPORTING INFORMATION}

reaction was initiated by addition of $30 \mu$ of $20 \mathrm{nM}$ PRMT5:MEP50. The final concentrations of the reaction mixture contained $1 \mu \mathrm{M} \mathrm{SAM}, 0.3 \mu \mathrm{M} \mathrm{H} 2 \mathrm{~A}$ and $10 \mathrm{nM}$ PRMT5:MEP50. The reaction mixture was incubated at $30{ }^{\circ} \mathrm{C}$ for $60 \mathrm{~min}$ and then quenched by adding $10 \mu \mathrm{l}$ formic acid solution (final concentration 1\%). Inhibition of SAH formation in the presence of compound was calculated as a percentage of the control relative to the uninhibited reaction as a function of inhibitor concentrations. Data were fit to the following equation

$$
y=\frac{100}{1+\left(\frac{x}{I C_{50}}\right)^{h}}
$$

$\mathrm{Y}$ is the percent of inhibition, $\mathrm{X}$ is $\log$ of compound concentration, $I C_{50}$ is the inhibitor concentration at $50 \%$ inhibition, and $\mathrm{h}$ is the Hill slope.

For the compounds that reach the assay limit (of $I C_{50} \leq 5 \mathrm{nM}$ ), compound potency was measured by reducing the enzyme concentration to $1.25 \mathrm{nM}$ with incubation time extended to $120 \mathrm{~min}$. 
SUPPORTING INFORMATION

Copies of ${ }^{1} \mathrm{H}$ and ${ }^{13} \mathrm{C}$ NMR spectra of new compounds

Frequency: $400 \mathrm{MHz}$
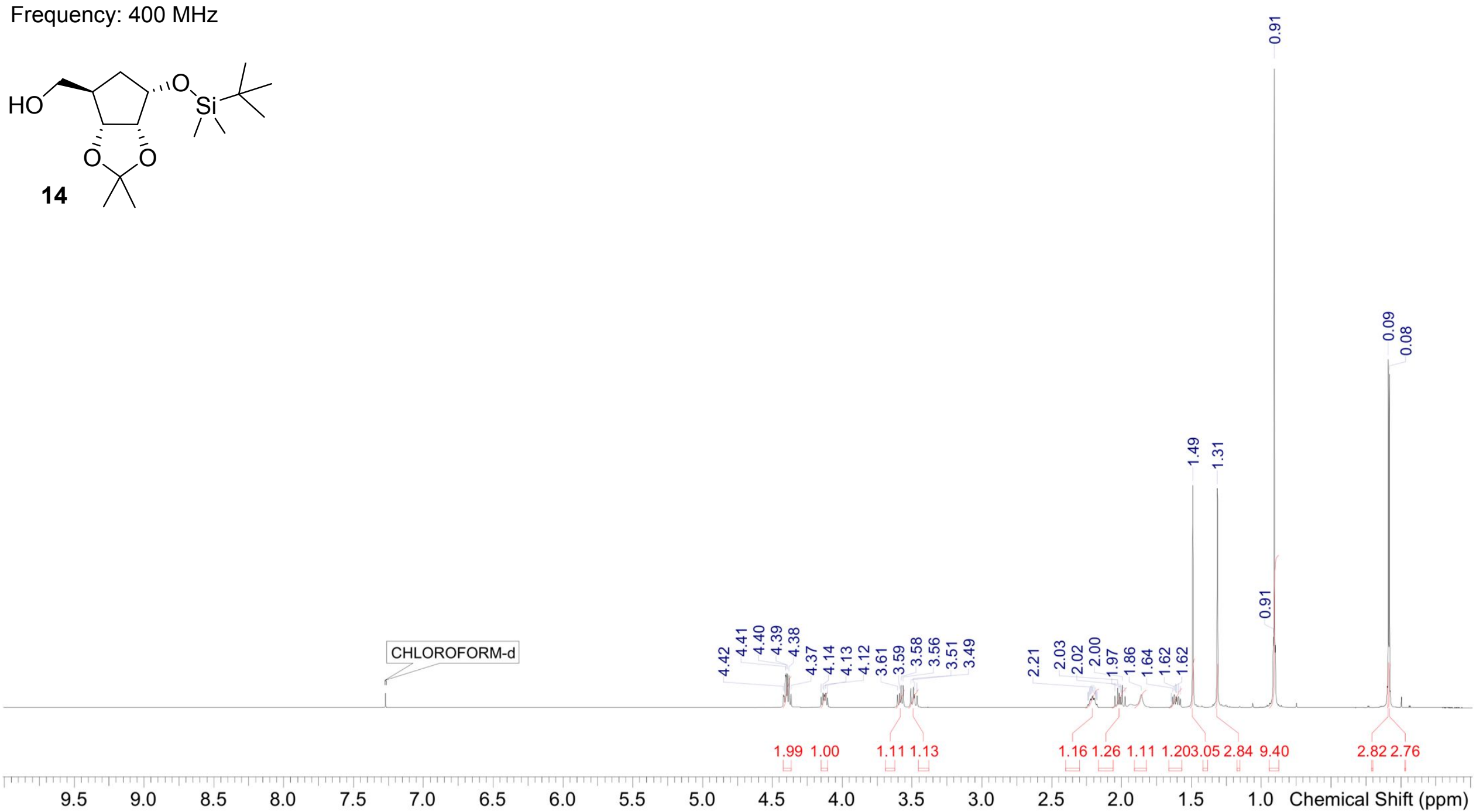


\section{SUPPORTING INFORMATION}

Frequency: $101 \mathrm{MHz}$

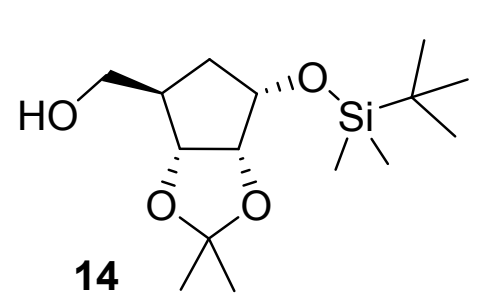

a

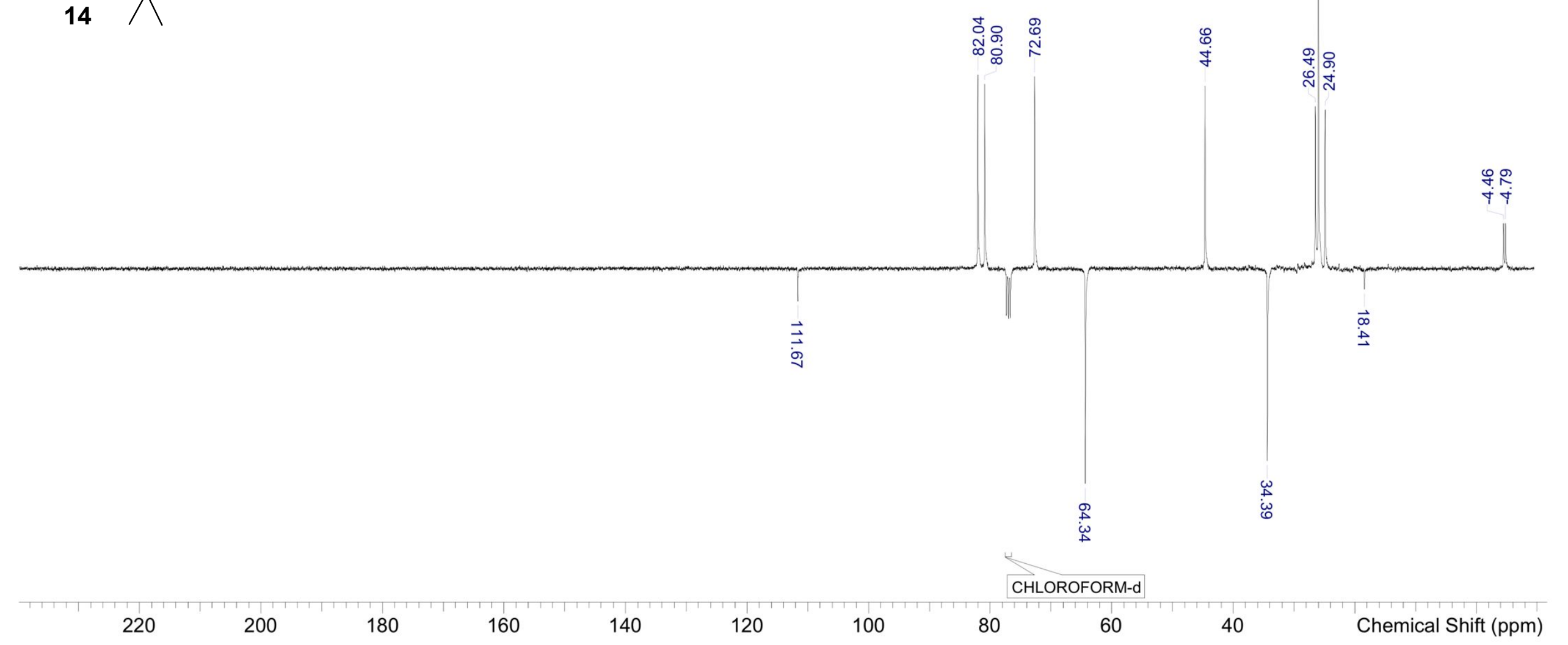




\section{SUPPORTING INFORMATION}

Frequency: $400 \mathrm{MHz}$

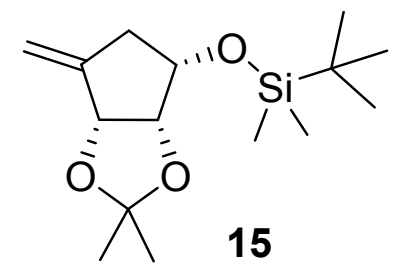

15

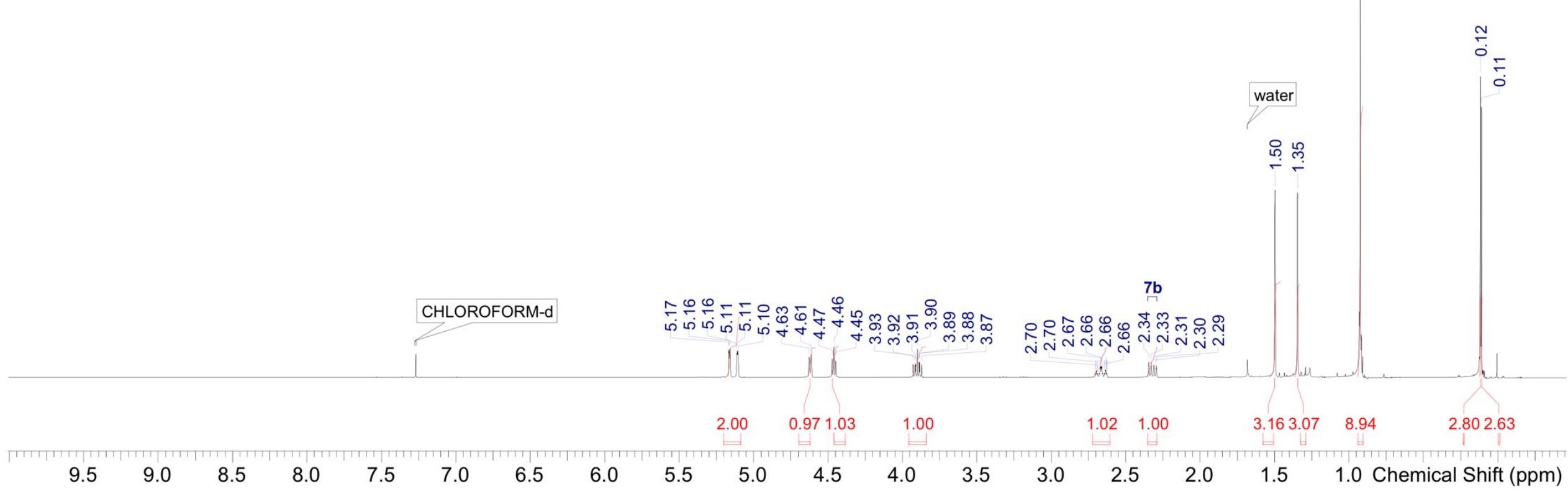




\section{SUPPORTING INFORMATION}

Frequency: $101 \mathrm{MHz}$

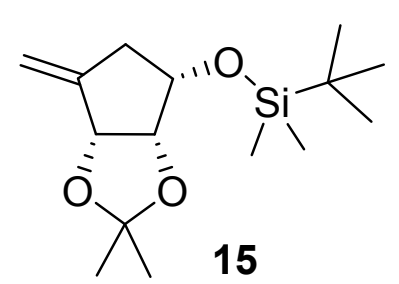

N

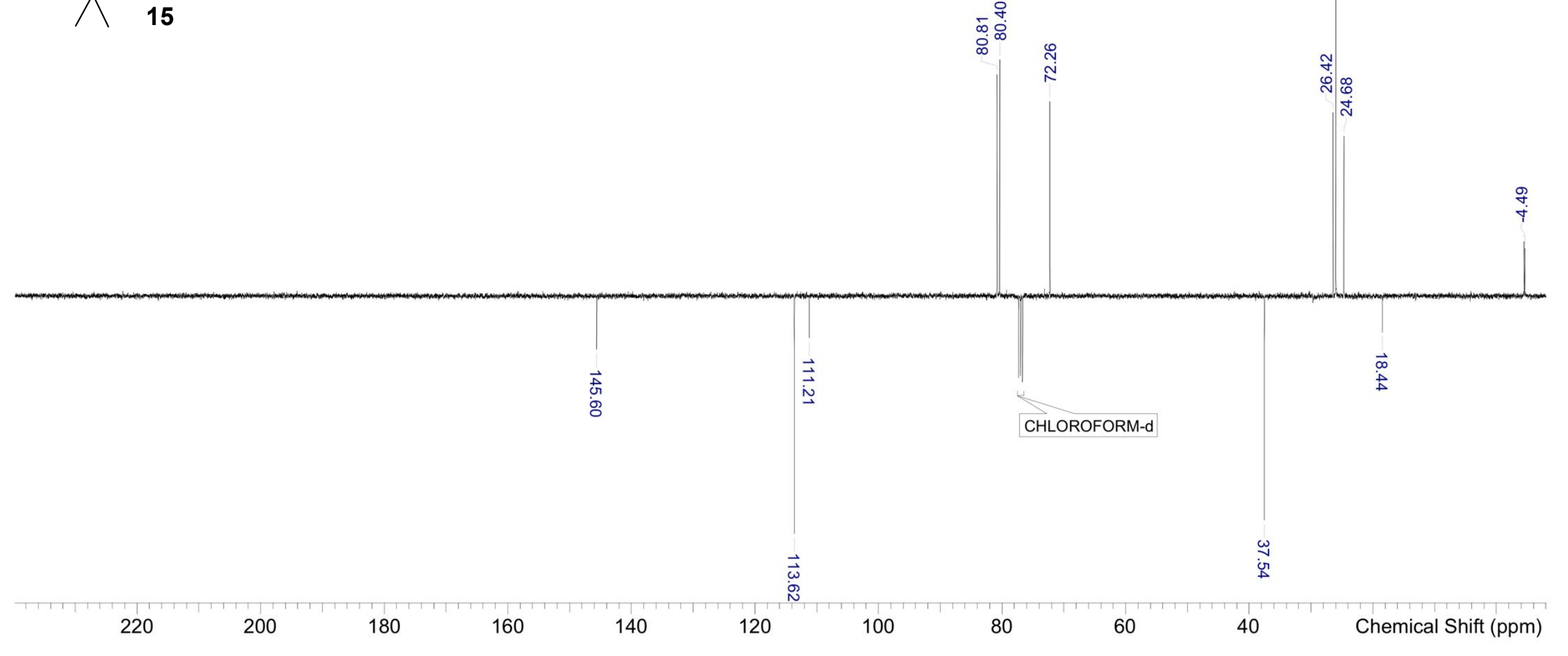




\section{SUPPORTING INFORMATION}

Frequency: $400 \mathrm{MHz}$
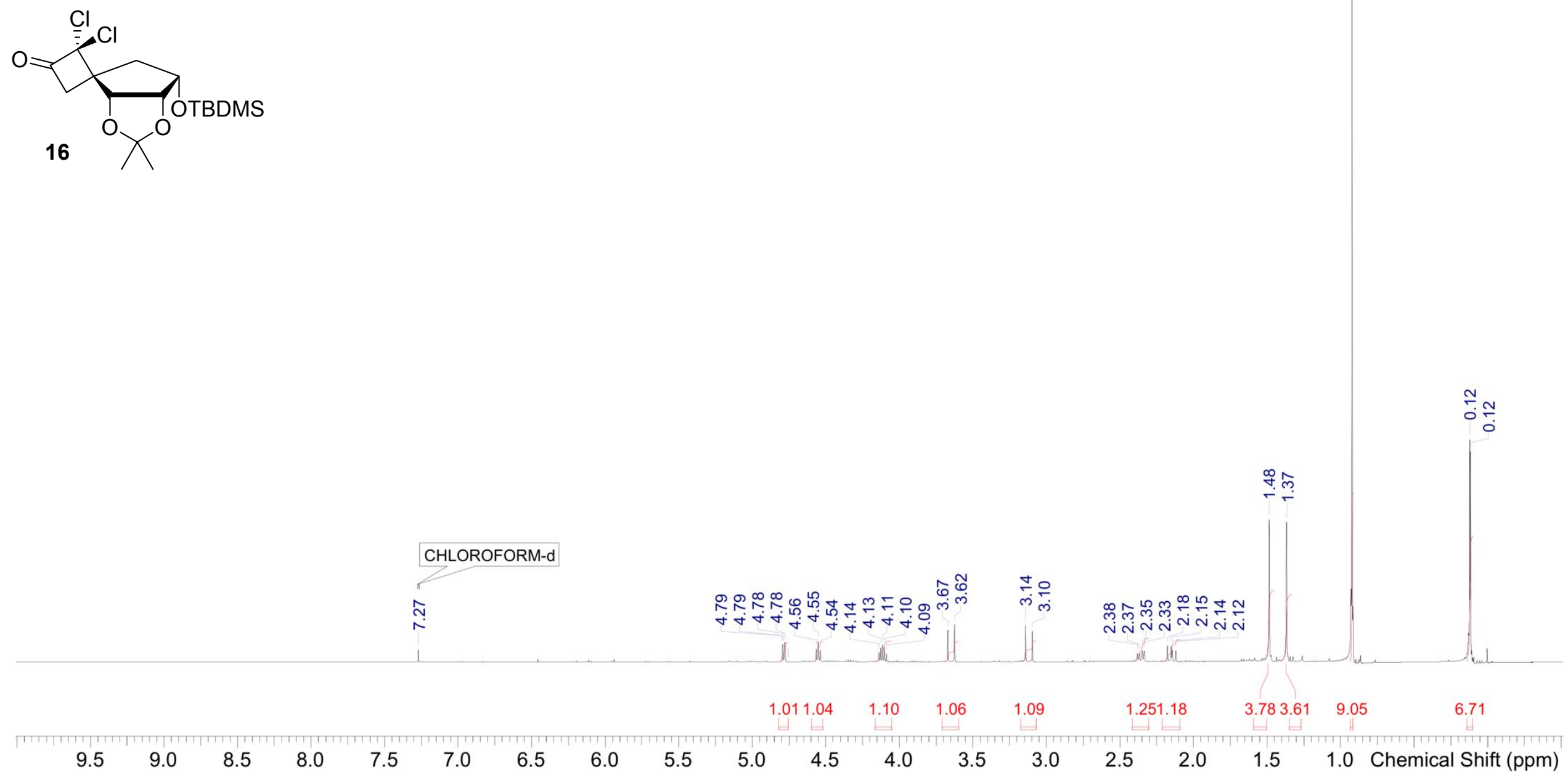
SUPPORTING INFORMATION

Frequency: $101 \mathrm{MHz}$
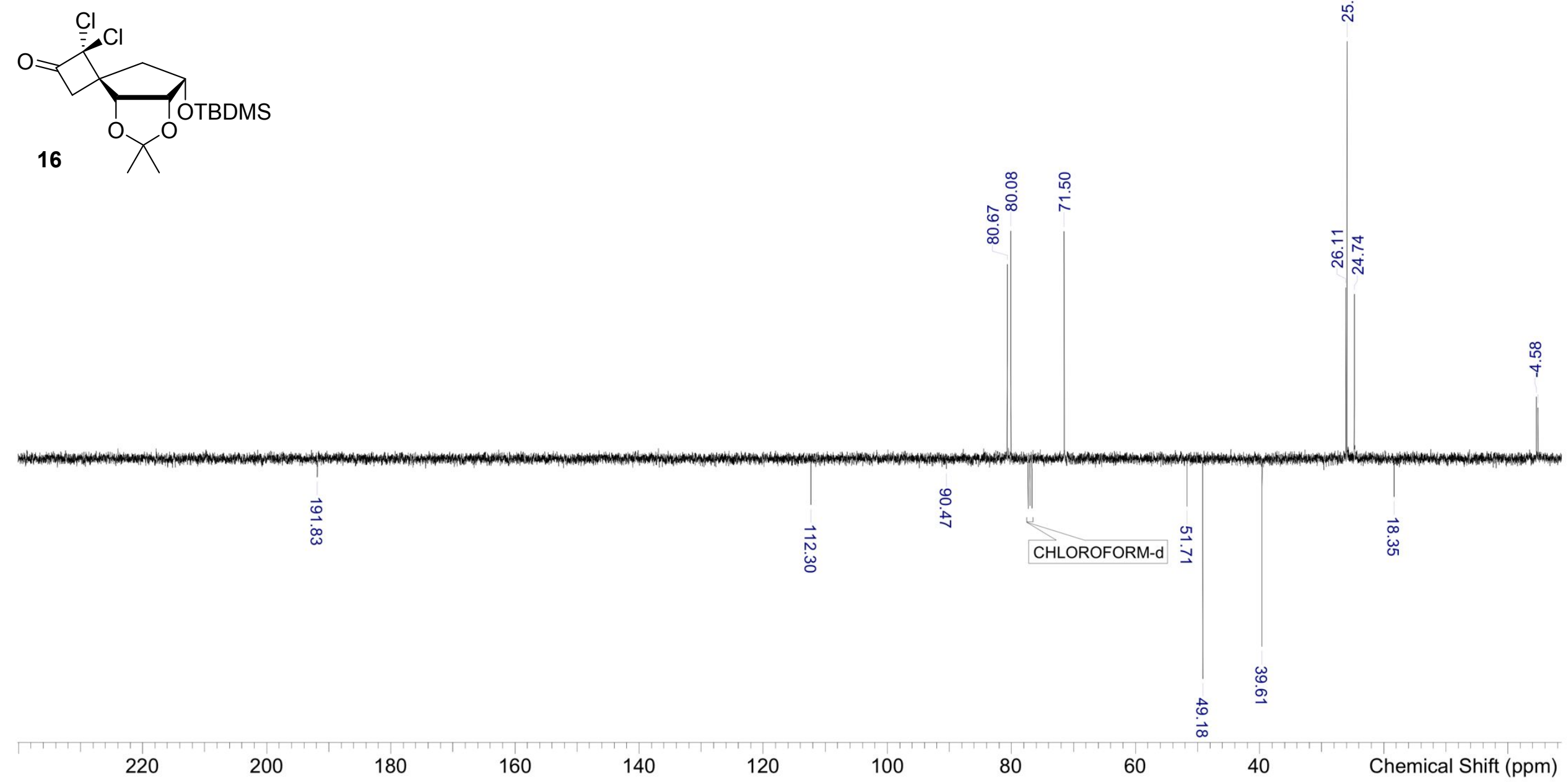


\section{SUPPORTING INFORMATION}

\section{Frequency: 400 MHz/ $101 \mathrm{MHz}$}
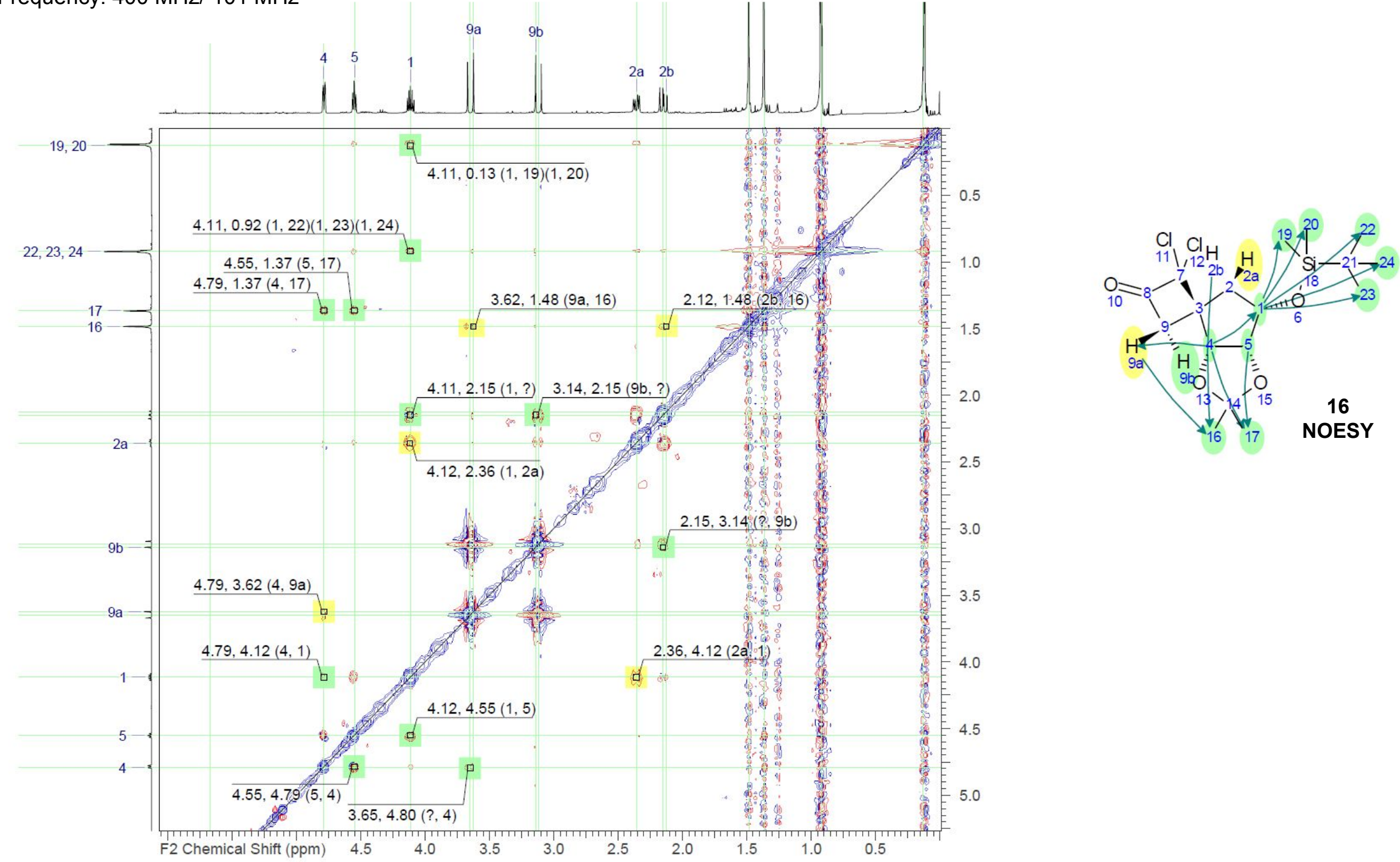


\section{SUPPORTING INFORMATION}

Frequency: $400 \mathrm{MHz}$

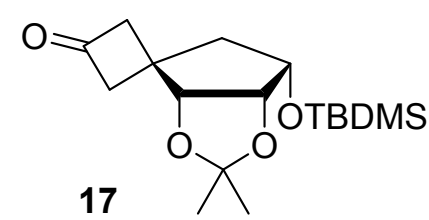

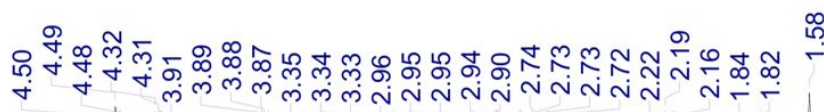

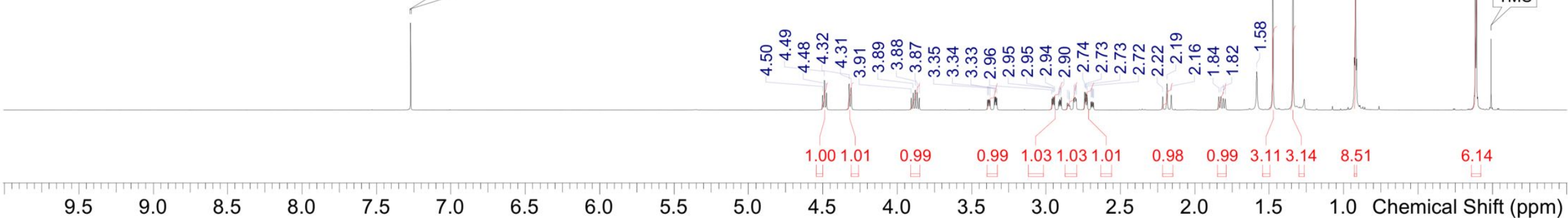




\section{SUPPORTING INFORMATION}

Frequency: $101 \mathrm{MHz}$

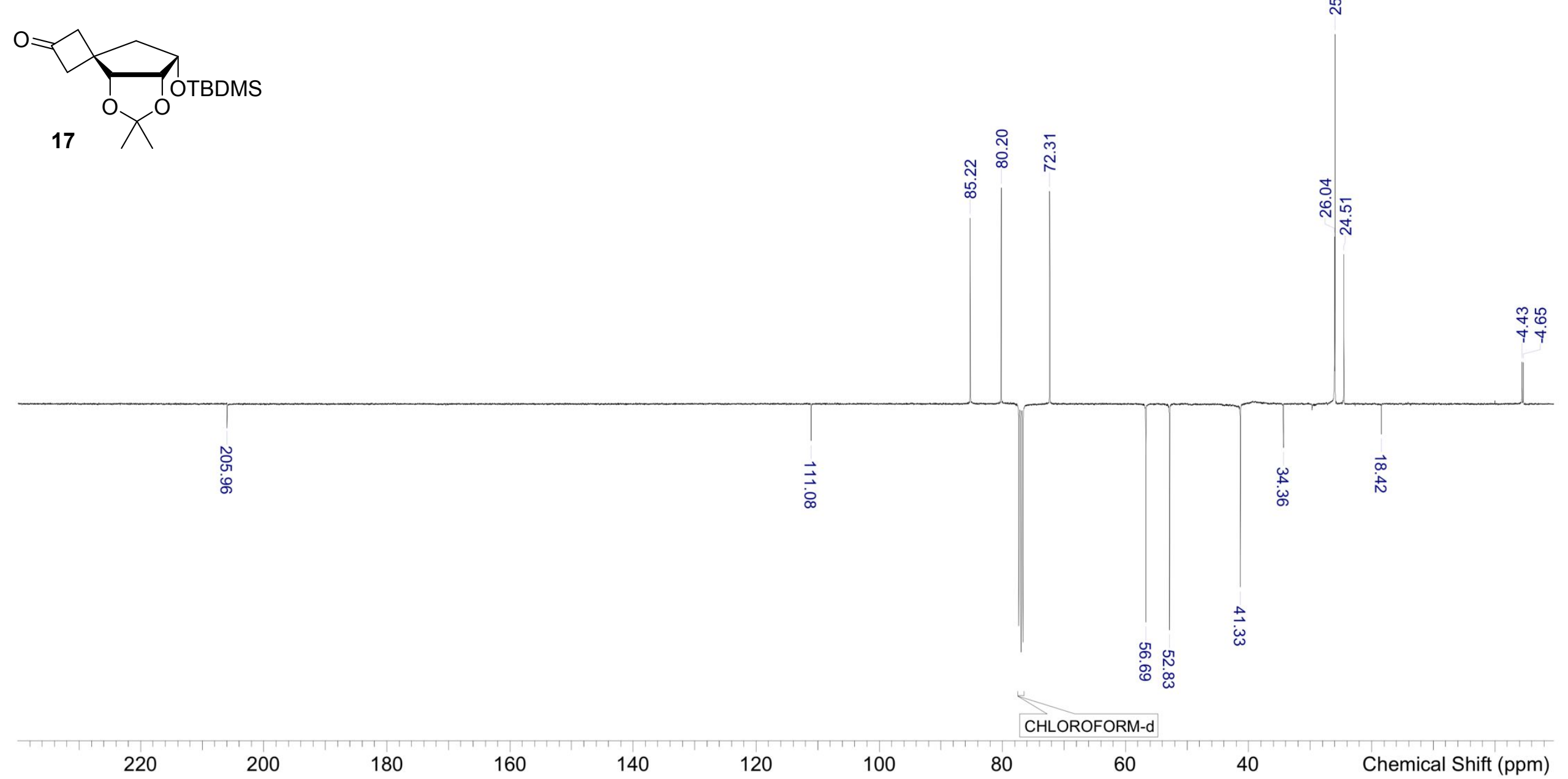




\section{SUPPORTING INFORMATION}

Frequency: $400 \mathrm{MHz}$

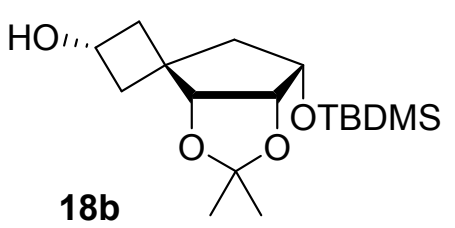

CHLOROFORM-d

ヘ

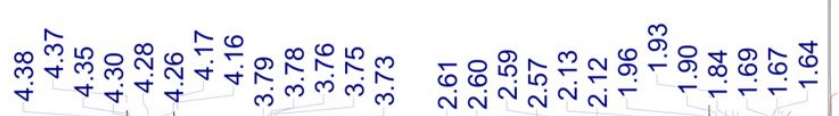
witat

1.021 .101 .071 .07 


\section{SUPPORTING INFORMATION}

Frequency: $101 \mathrm{MHz}$

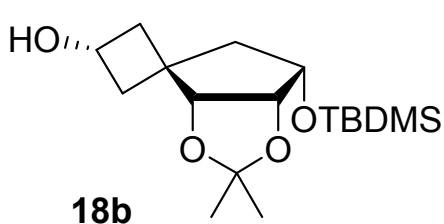

$18 b$

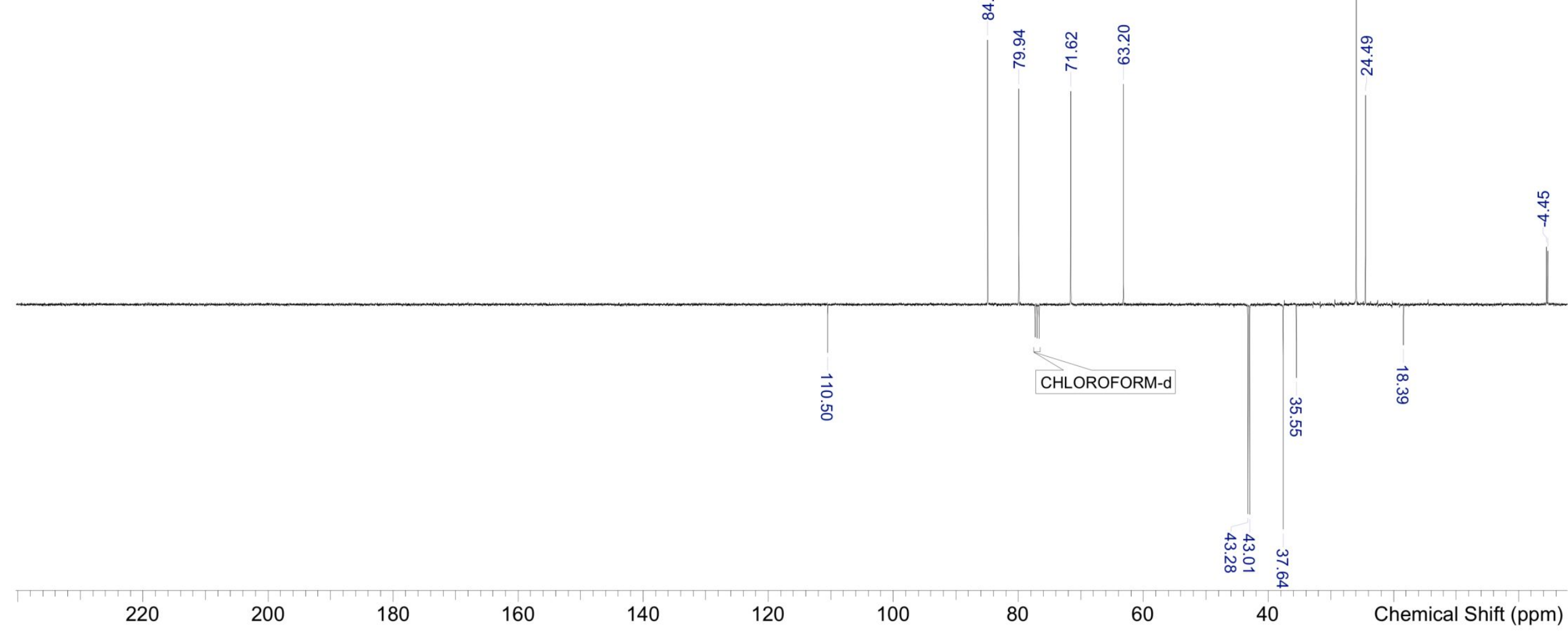




\section{SUPPORTING INFORMATION}

Frequency: $400 \mathrm{MHz}$

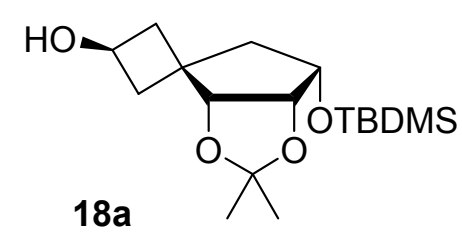

$18 a$

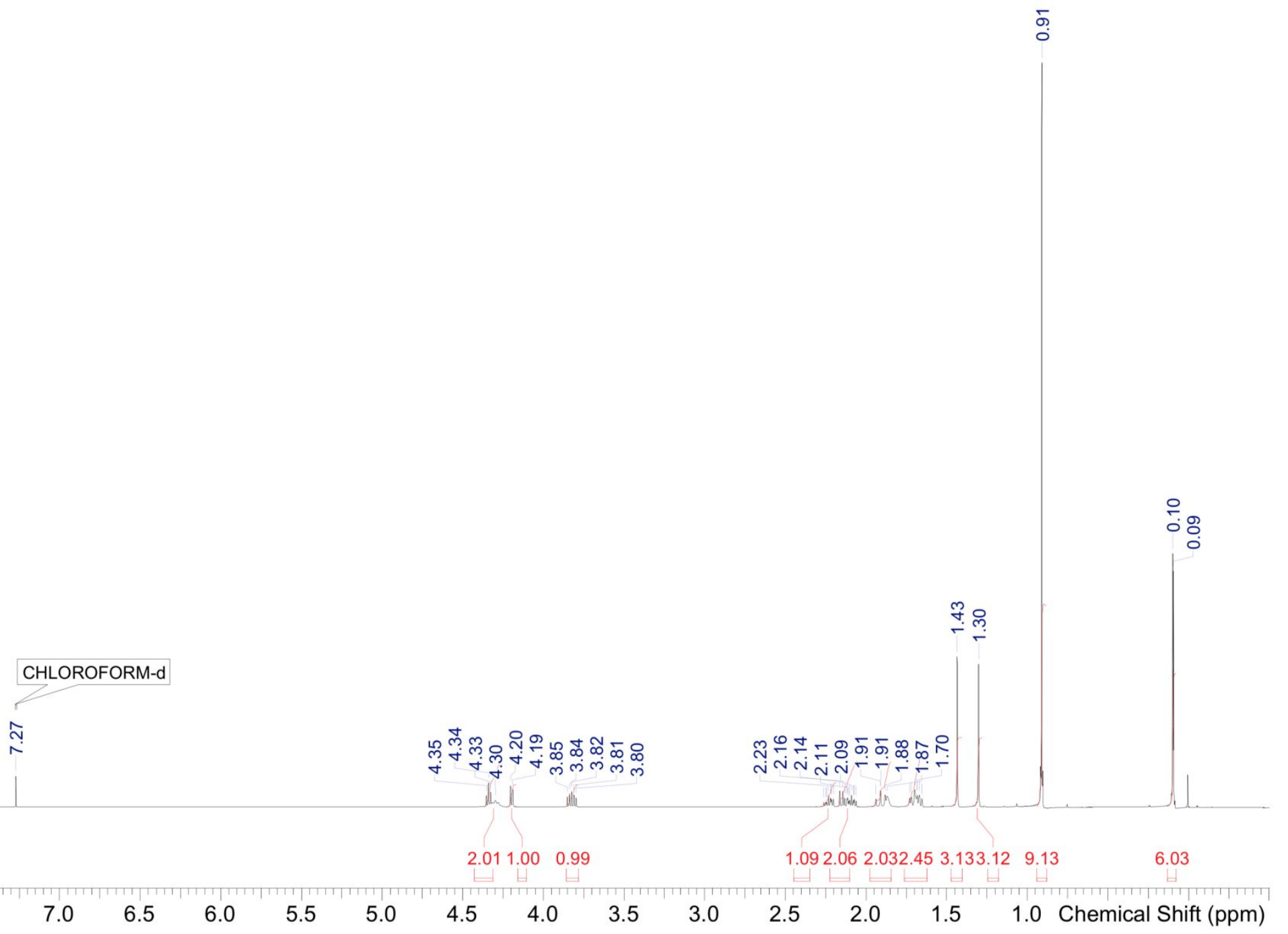




\section{SUPPORTING INFORMATION}

Frequency: $400 \mathrm{MHz}$

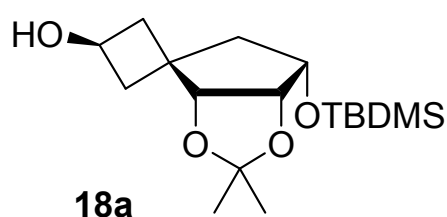

CHLOROFORM-d

กิ

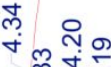



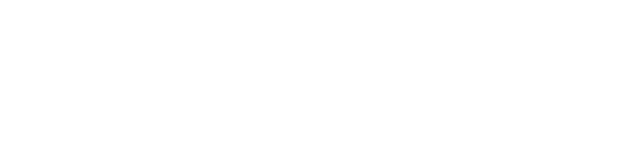

$1.092 .062 .032 .453 .133 .12 \quad 9.13$ 


\section{SUPPORTING INFORMATION}

Frequency: $101 \mathrm{MHz}$
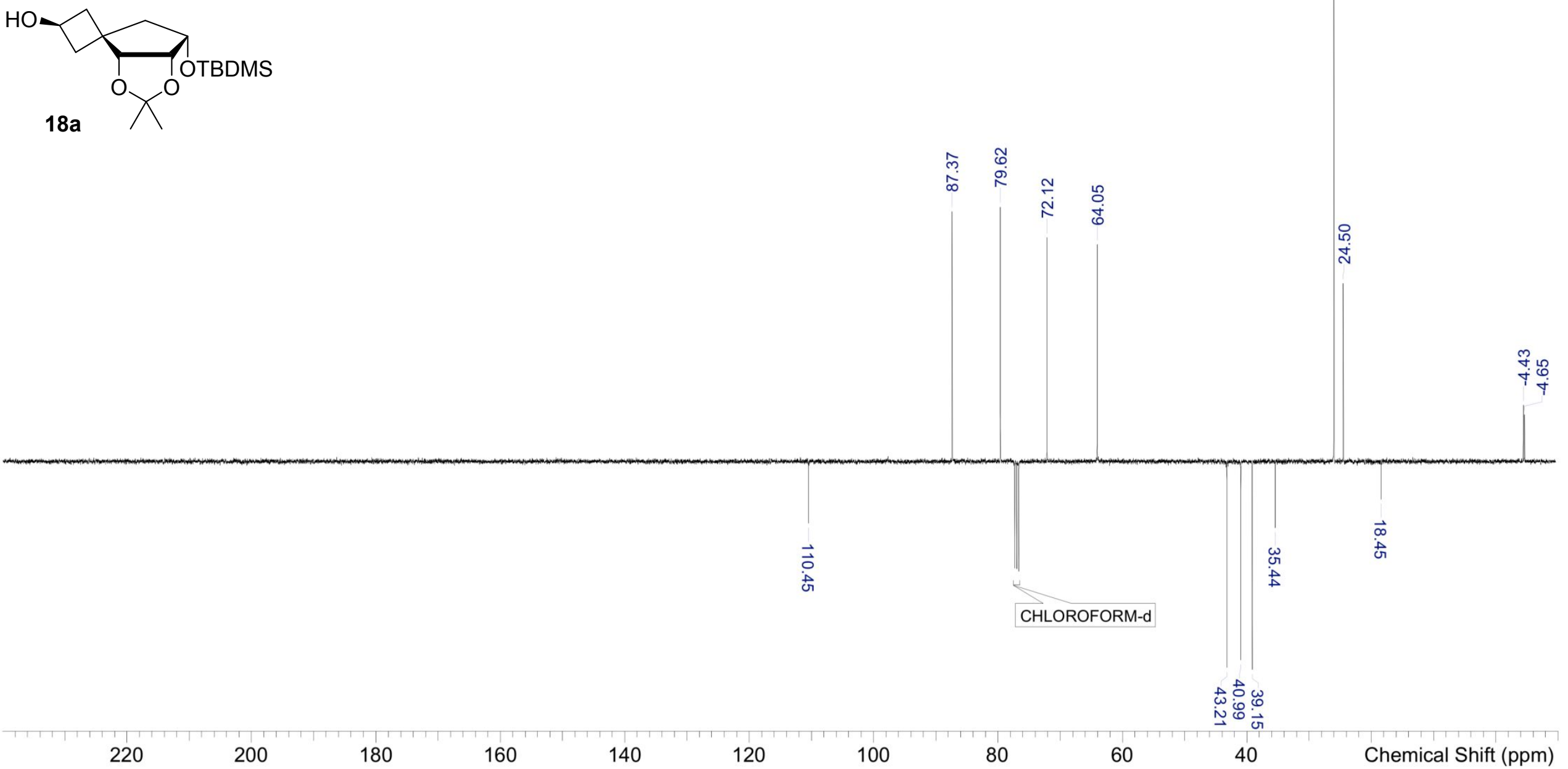


\section{SUPPORTING INFORMATION}

Frequency: $400 \mathrm{MHz}$
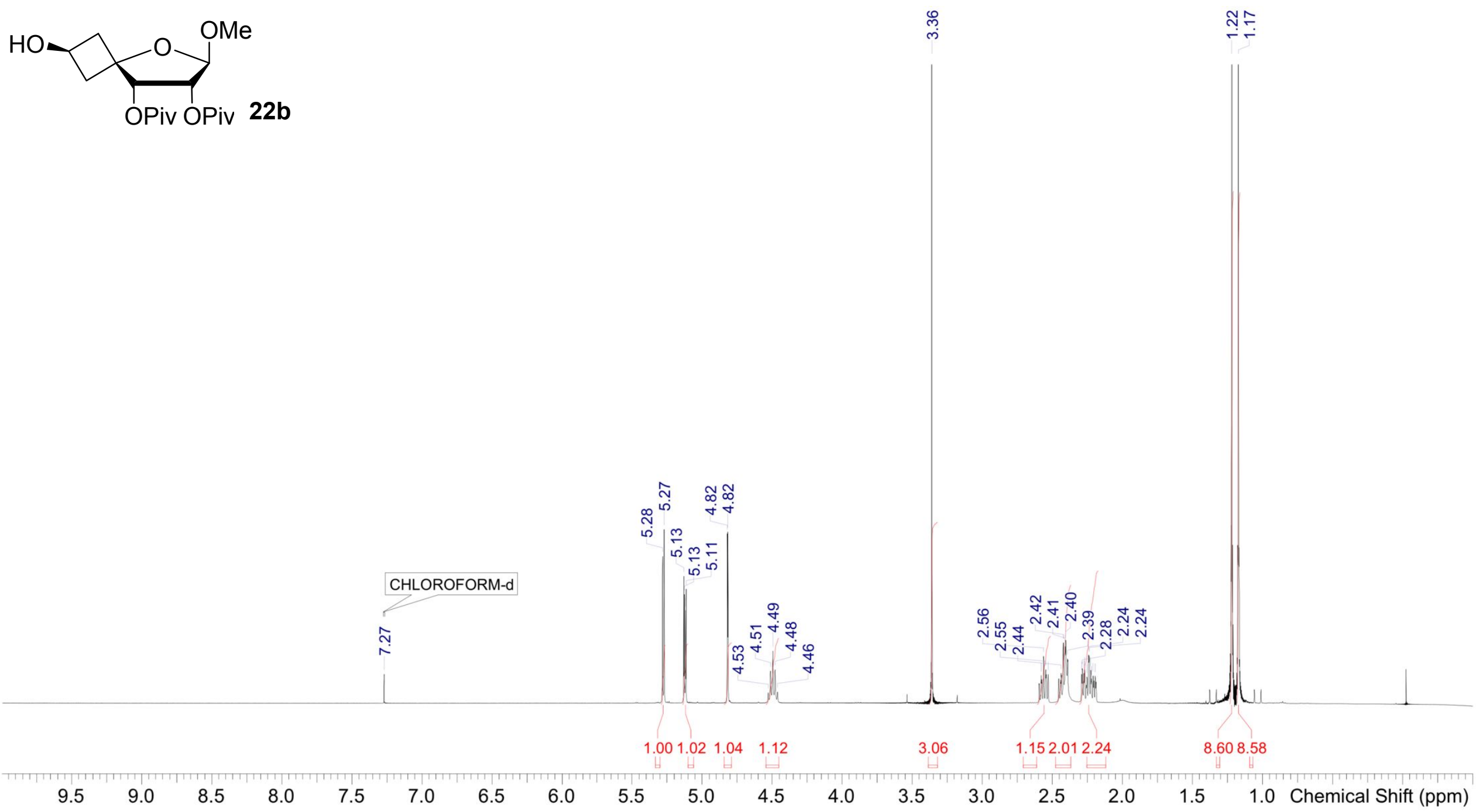


\section{SUPPORTING INFORMATION}

Frequency: $101 \mathrm{MHz}$
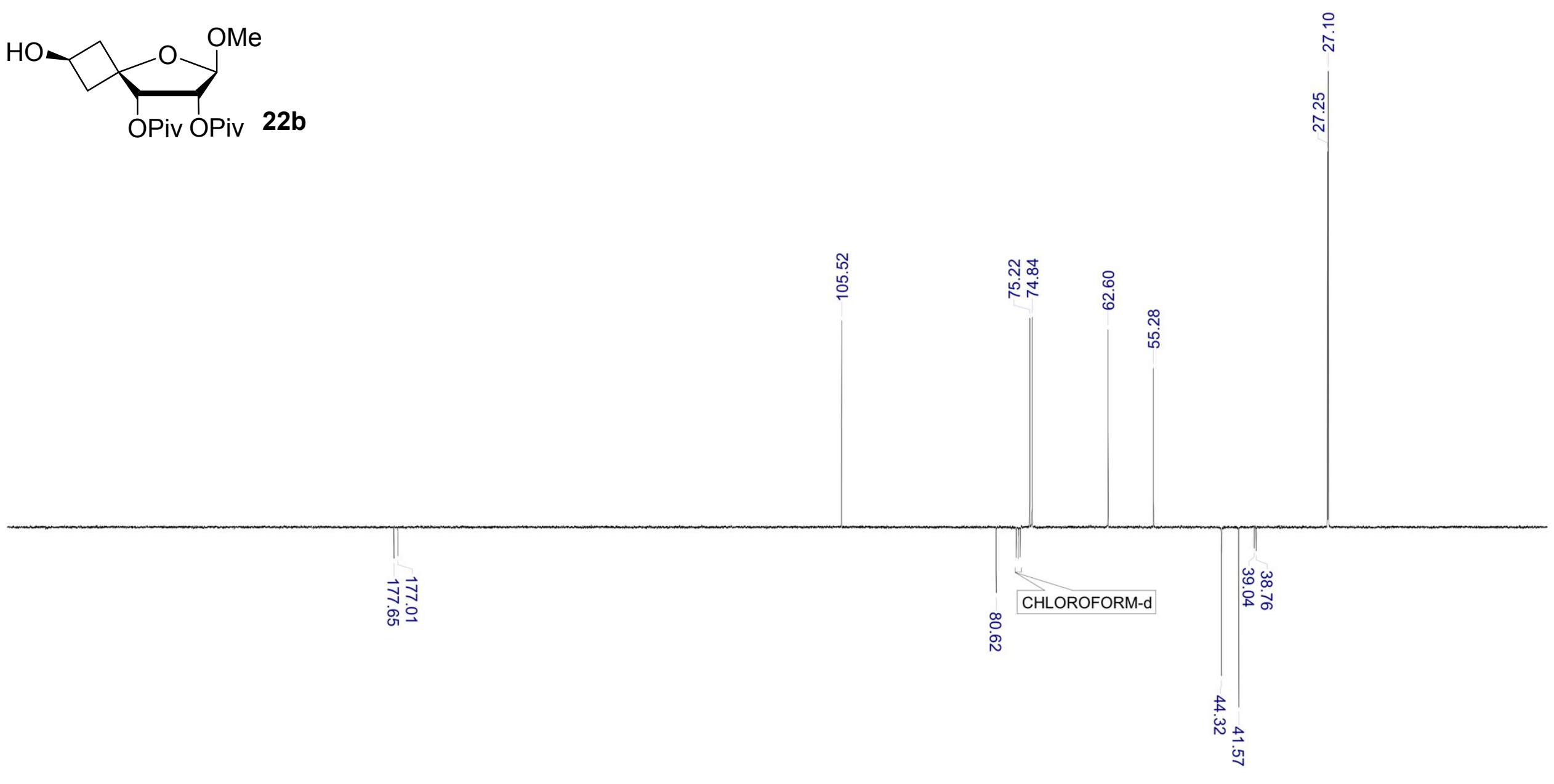


\section{SUPPORTING INFORMATION}

Frequency: $400 \mathrm{MHz}$

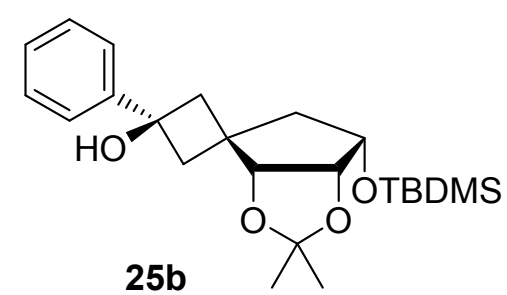

$25 b$

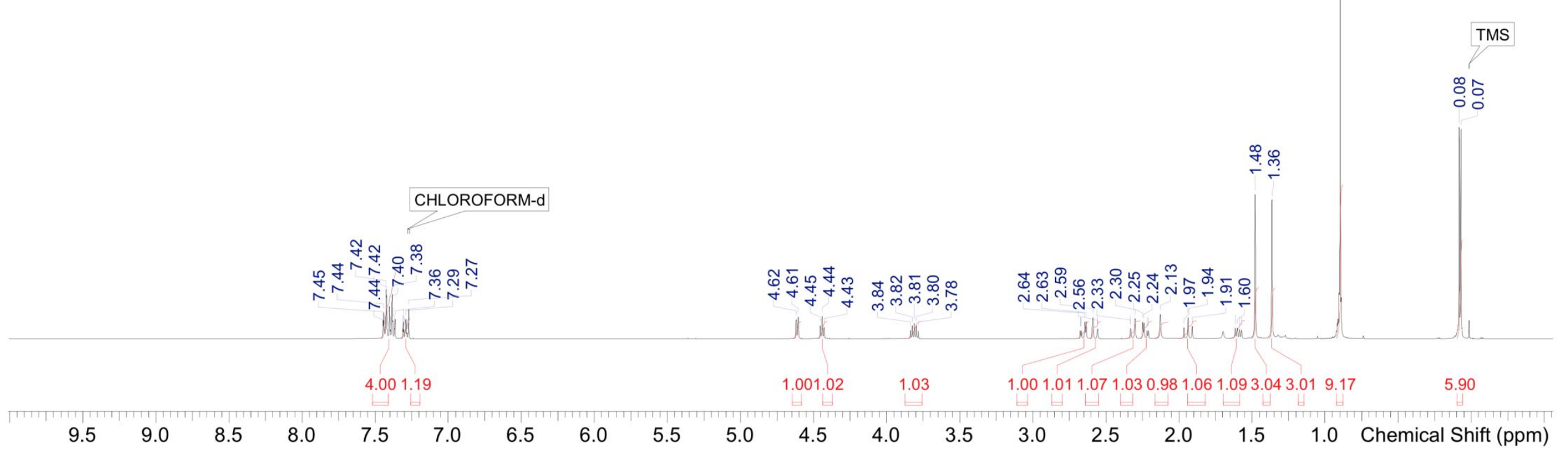




\section{SUPPORTING INFORMATION}

Frequency: $101 \mathrm{MHz}$

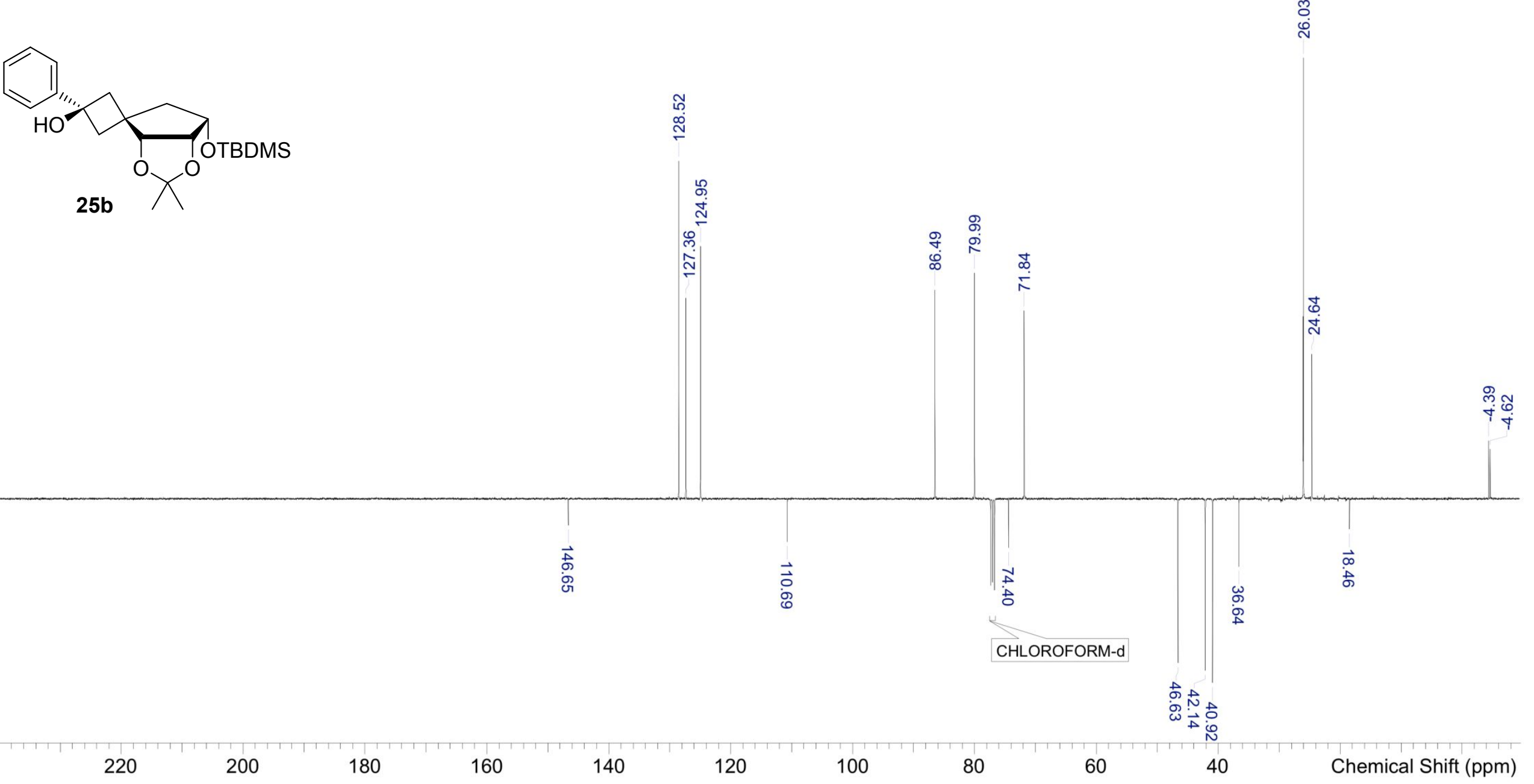




\section{SUPPORTING INFORMATION}

Frequency: $400 \mathrm{MHz}$

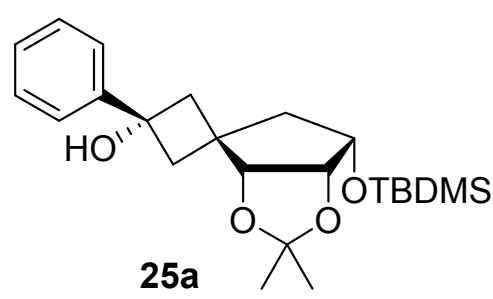

$25 a$

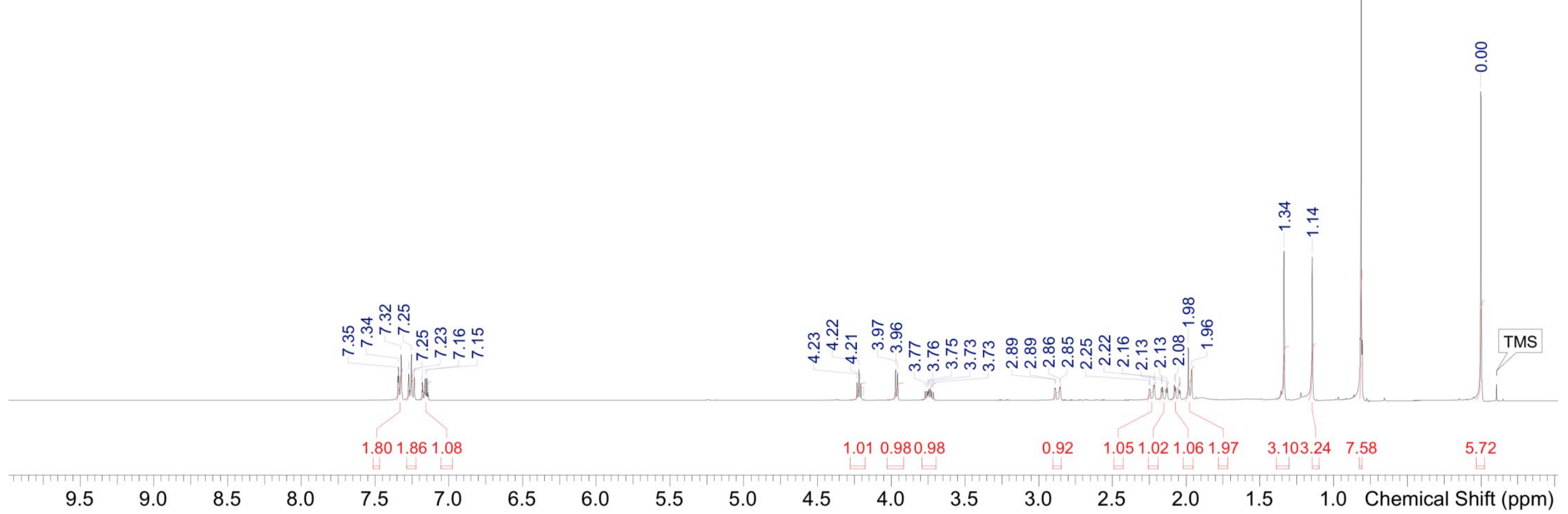




\section{SUPPORTING INFORMATION}

Frequency: $101 \mathrm{MHz}$

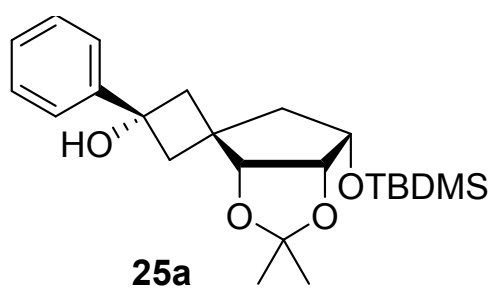

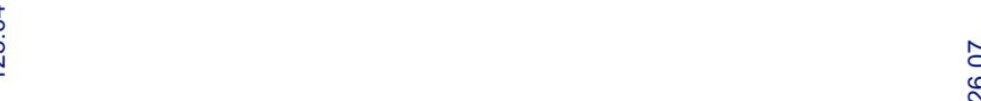

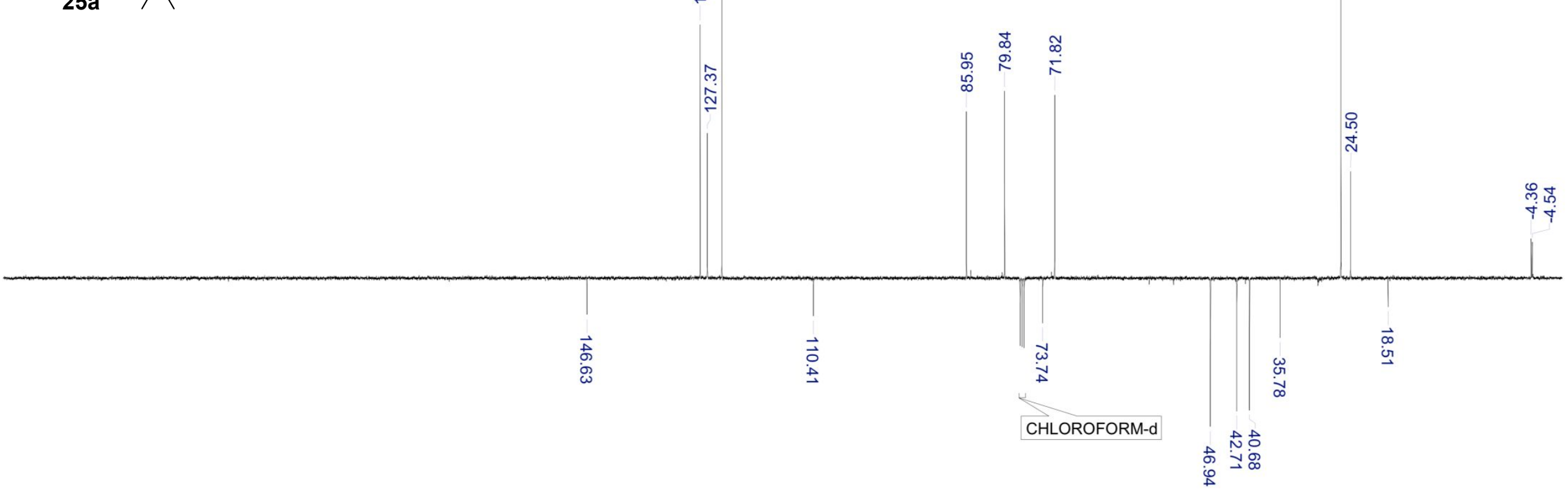




\section{SUPPORTING INFORMATION}

Frequency: $400 \mathrm{MHz}$

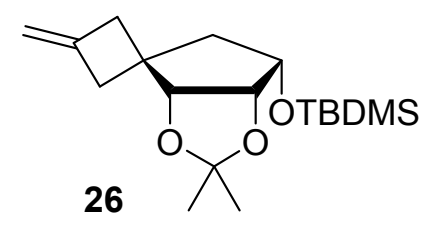

CHLOROFORM-d

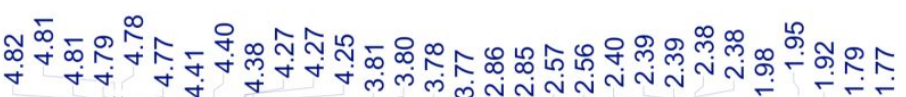
II

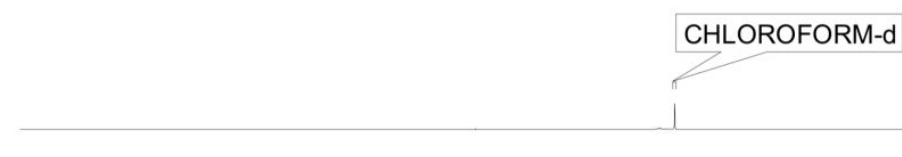

$\begin{array}{lllllll}1.02 & 0.490 .48 & 0.52 & 0.54 & 0.530 .85 & 0.530 .551 .521 .46 & 4.27\end{array}$

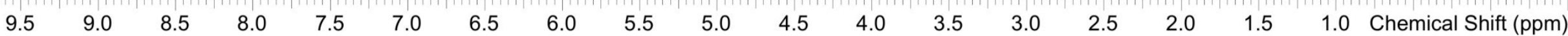




\section{SUPPORTING INFORMATION}

Frequency: $101 \mathrm{MHz}$
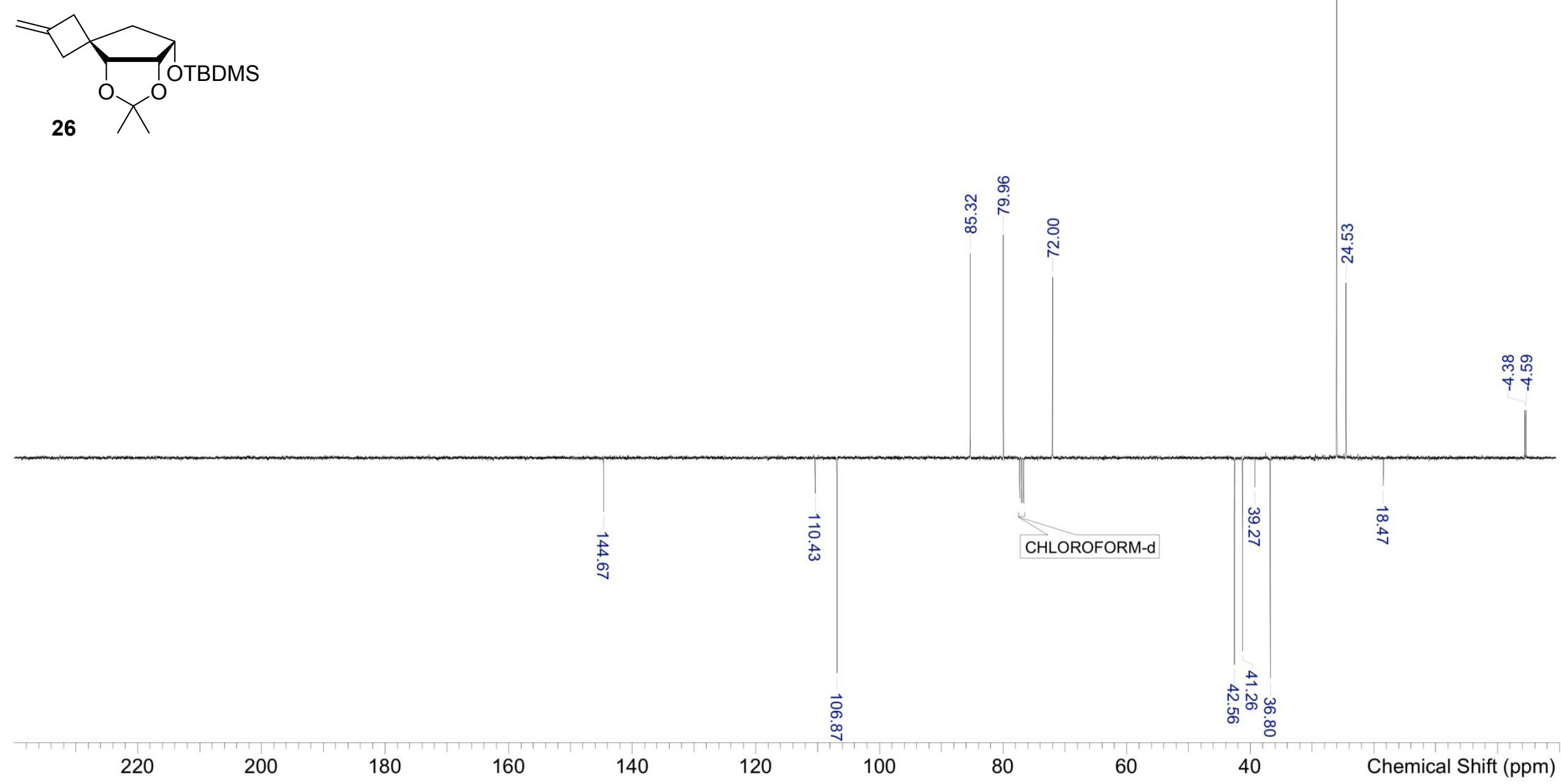


\section{SUPPORTING INFORMATION}

Frequency: $500 \mathrm{MHz}$
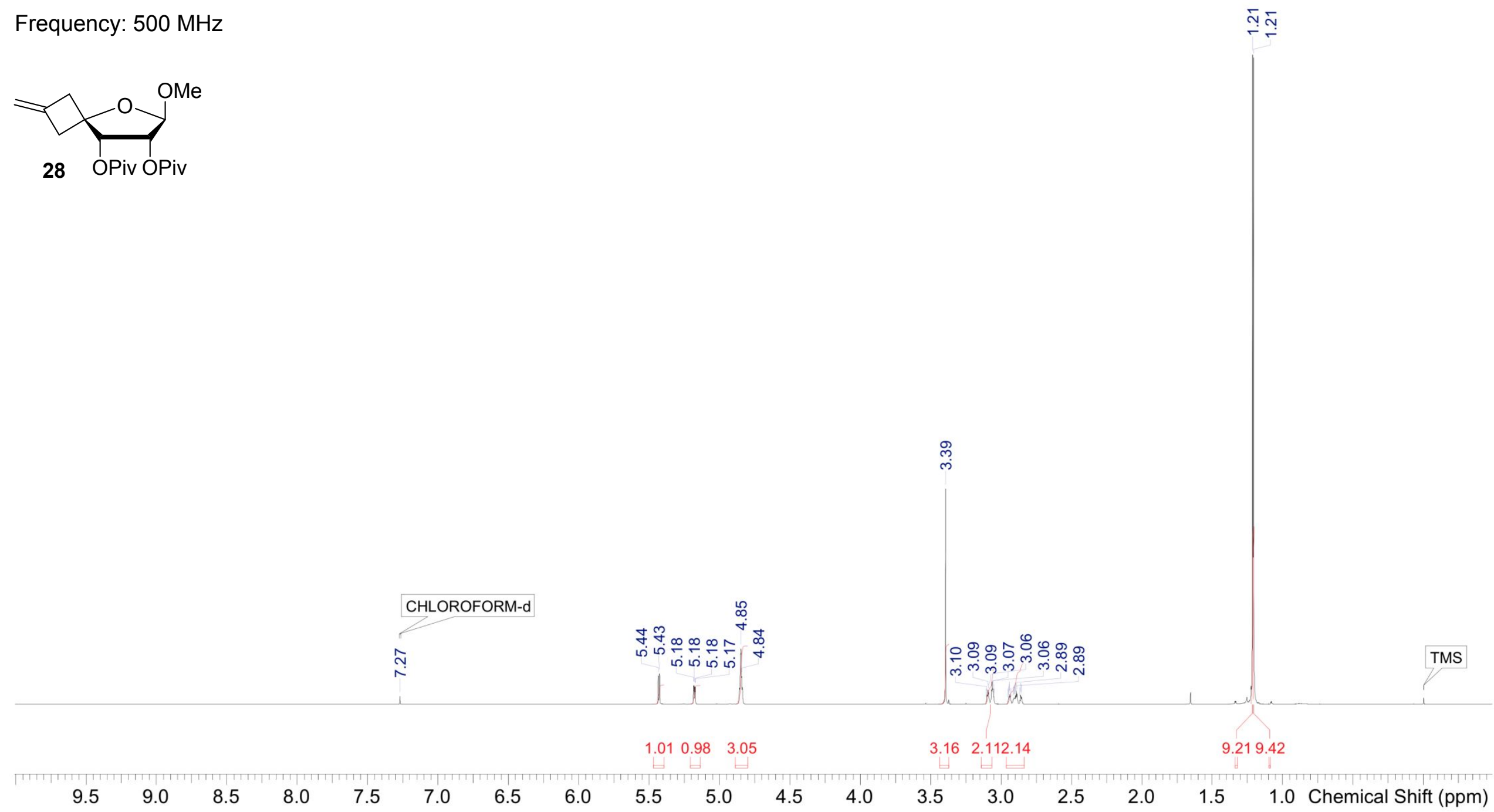


\section{SUPPORTING INFORMATION}

Frequency: $125 \mathrm{MHz}$

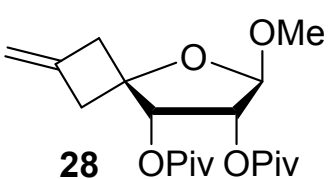

28 OPiv OPiv

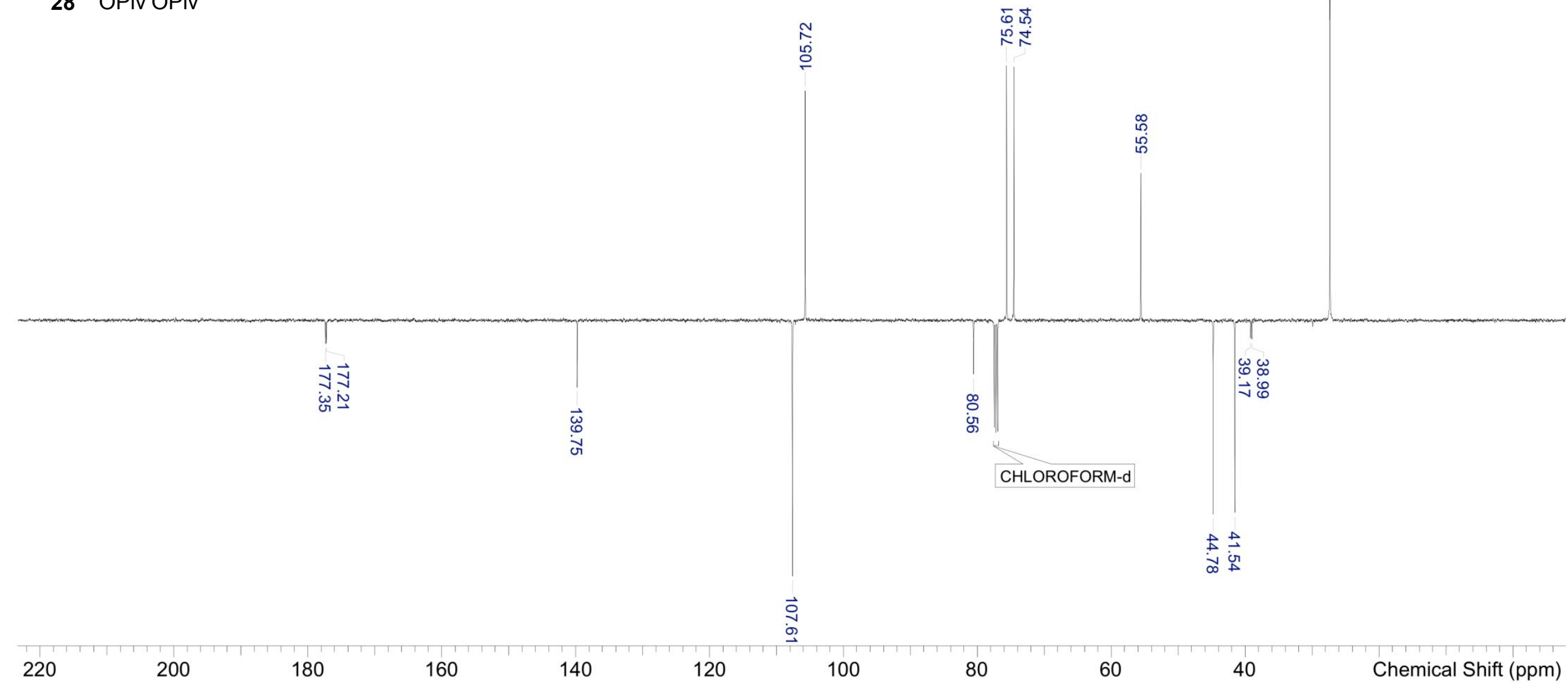




\section{SUPPORTING INFORMATION}

Frequency: $500 \mathrm{MHz}$

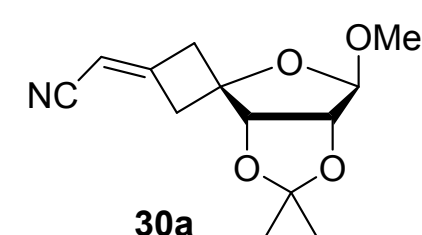

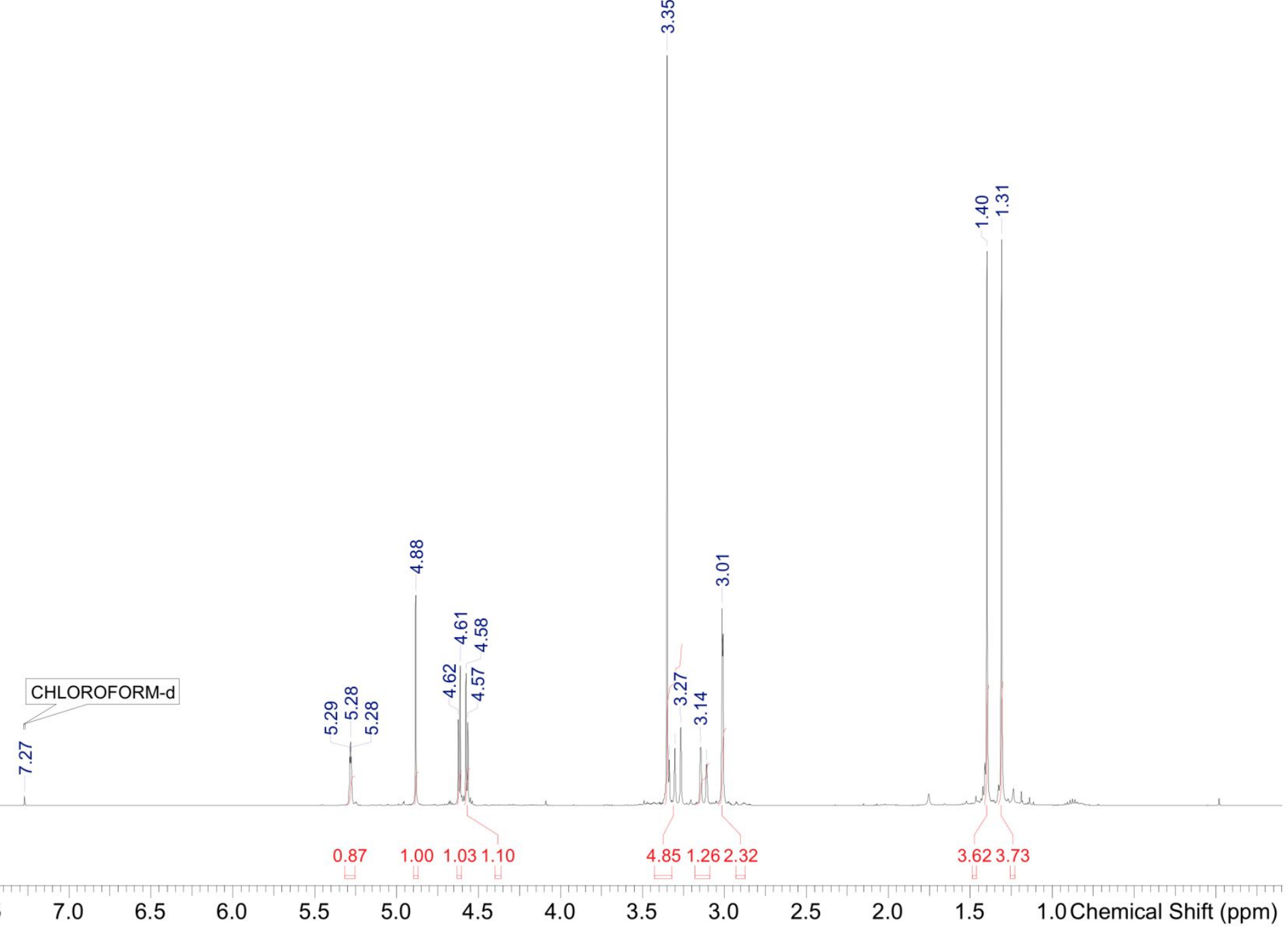




\section{SUPPORTING INFORMATION}

Frequency: $125 \mathrm{MHz}$
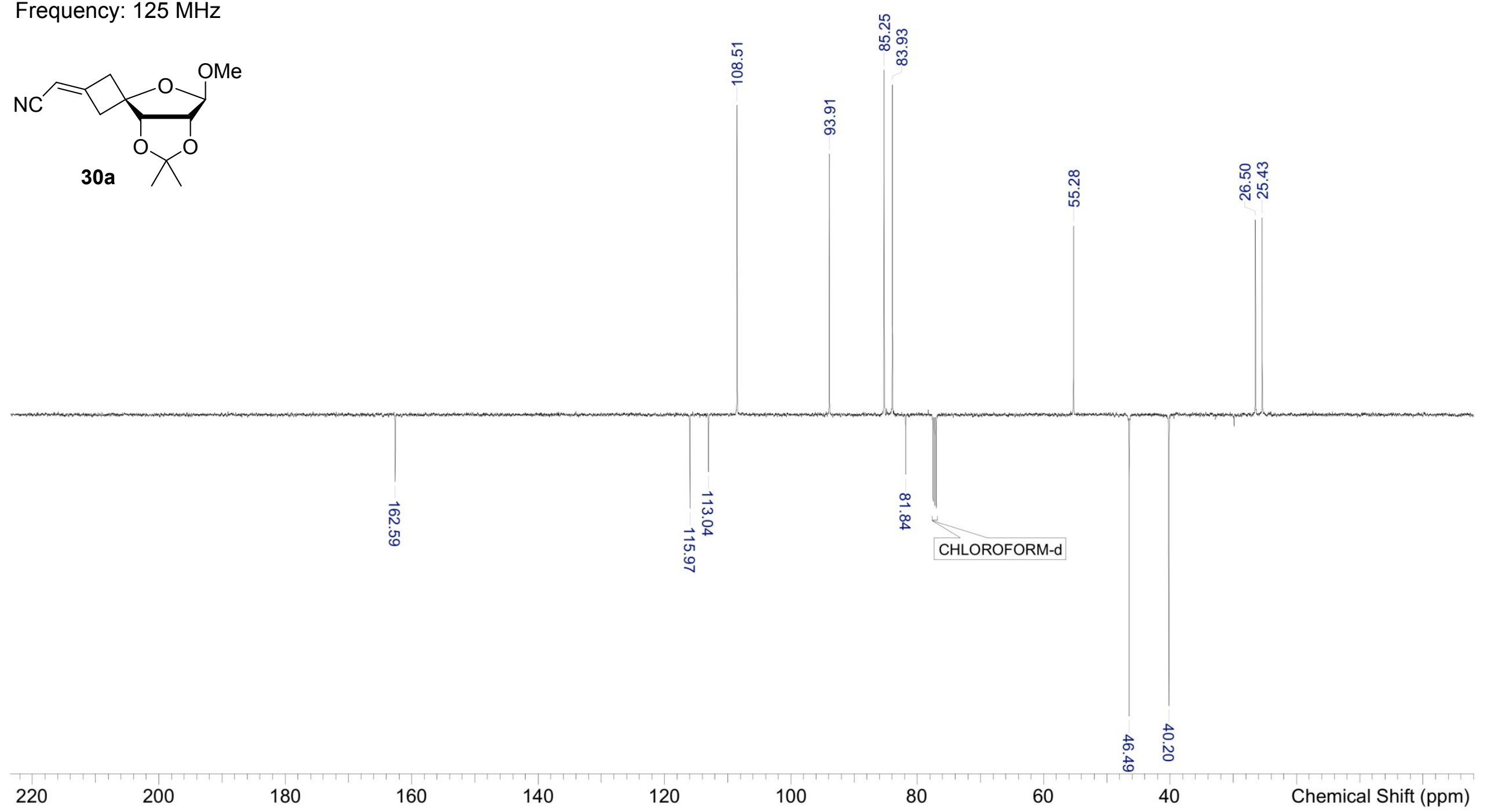


\section{SUPPORTING INFORMATION}

\section{Frequency: $500 \mathrm{MHz}$}
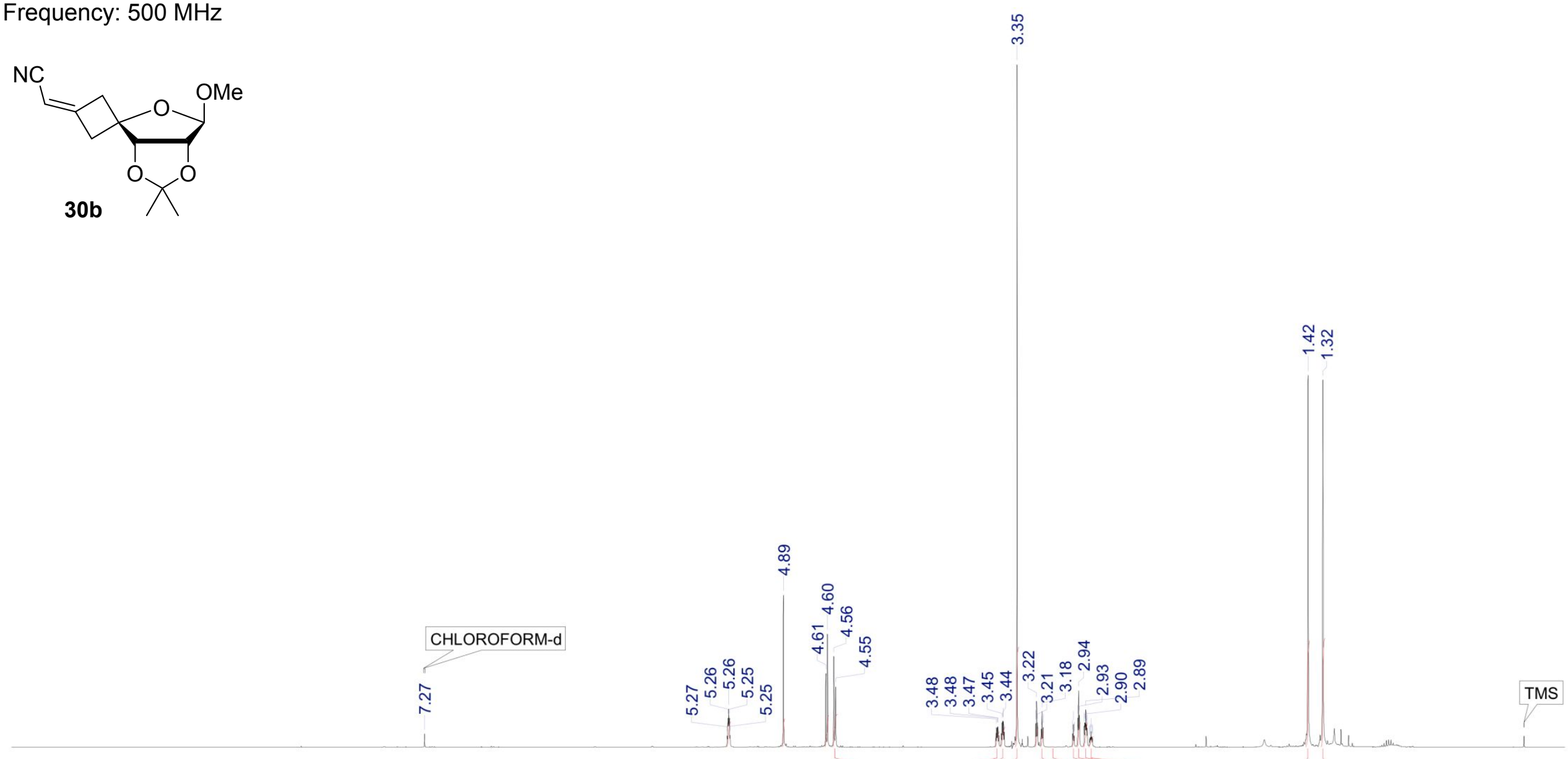

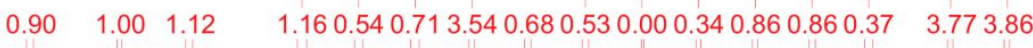




\section{SUPPORTING INFORMATION}

\section{Frequency: $125 \mathrm{MHz}$}
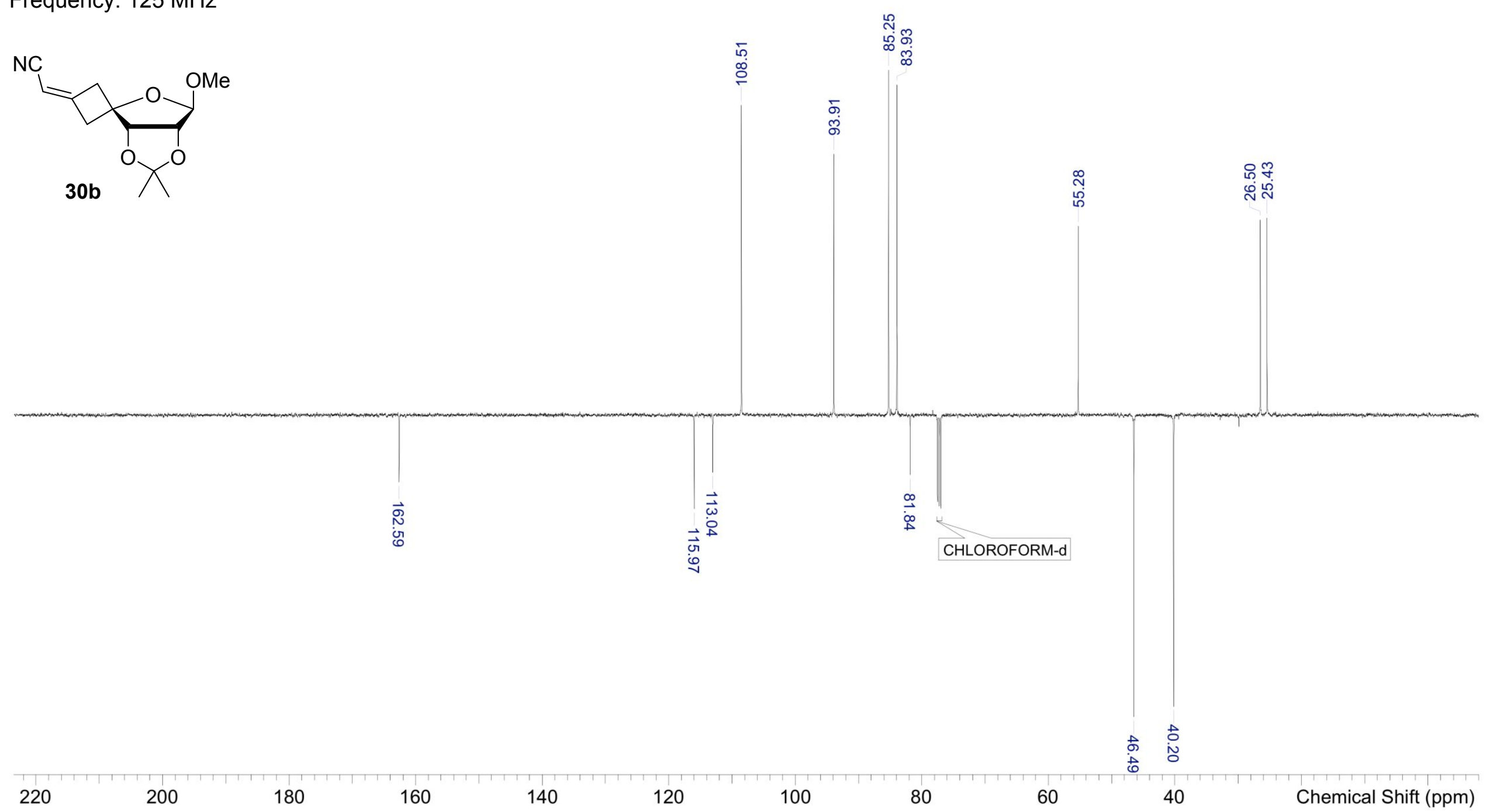


\section{SUPPORTING INFORMATION}

\section{Frequency: $500 \mathrm{MHz}$}
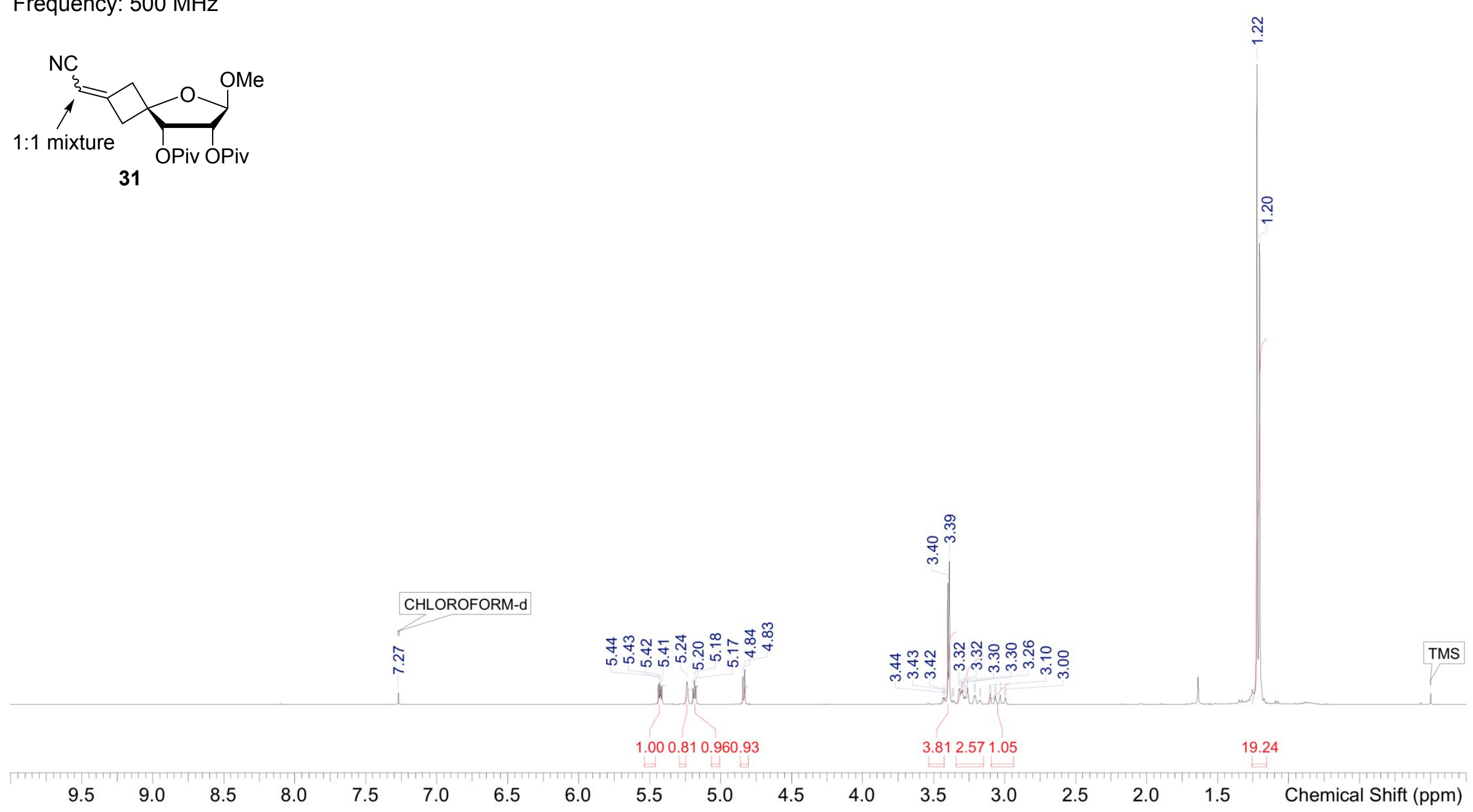


\section{SUPPORTING INFORMATION}

Frequency: $125 \mathrm{MHz}$

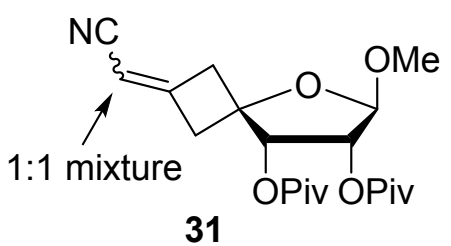

31

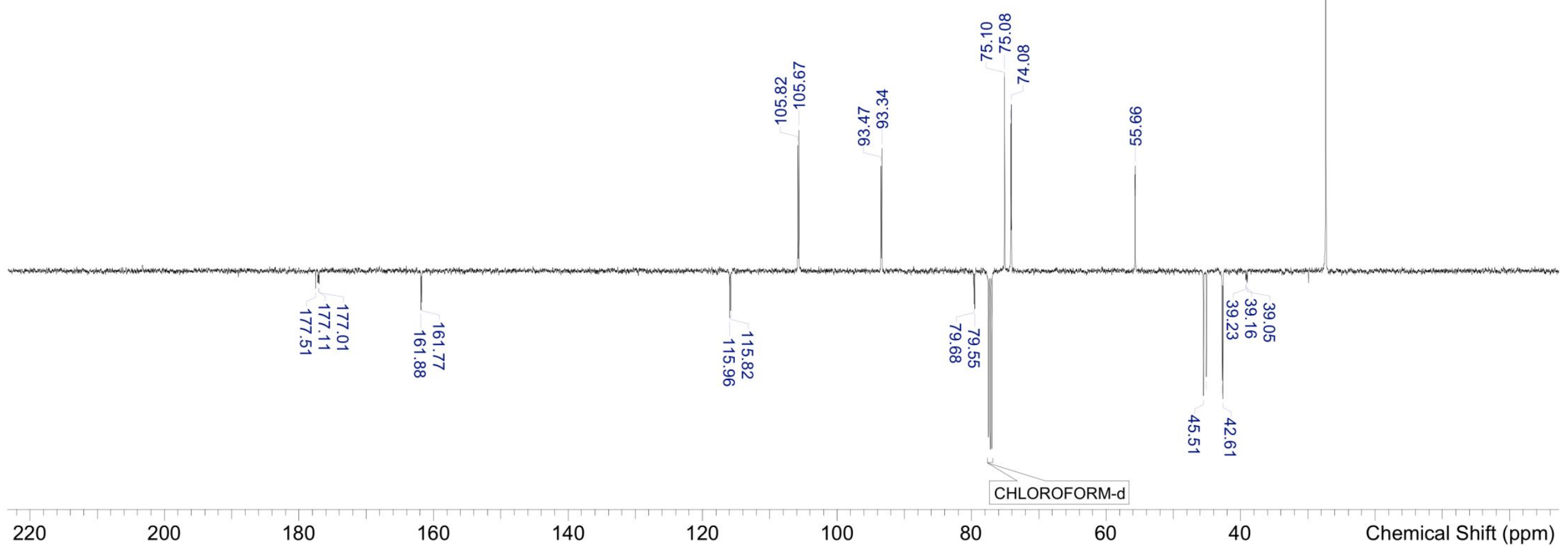




\section{SUPPORTING INFORMATION}

Frequency: $400 \mathrm{MHz}$
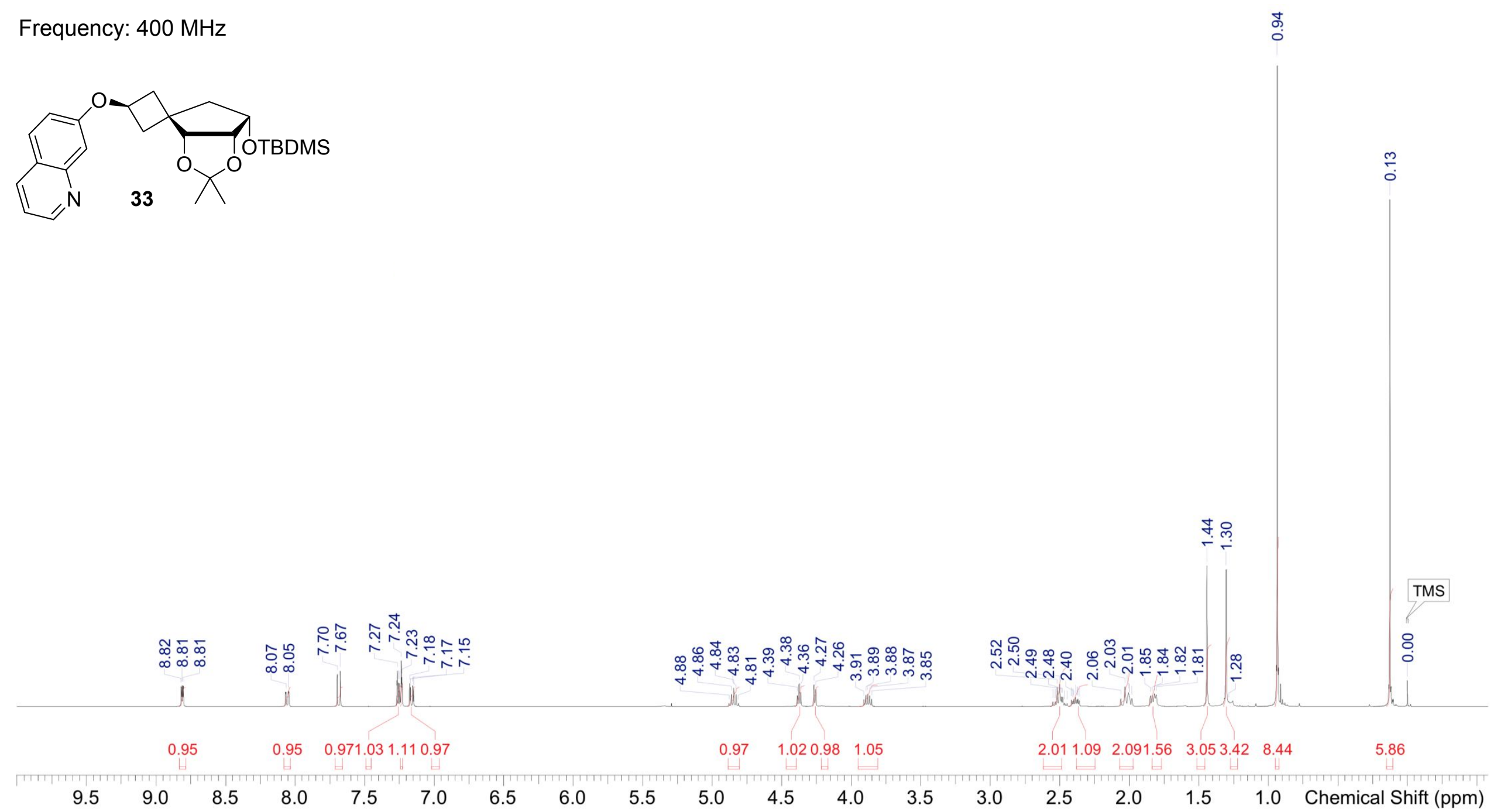


\section{SUPPORTING INFORMATION}

Frequency: $101 \mathrm{MHz}$
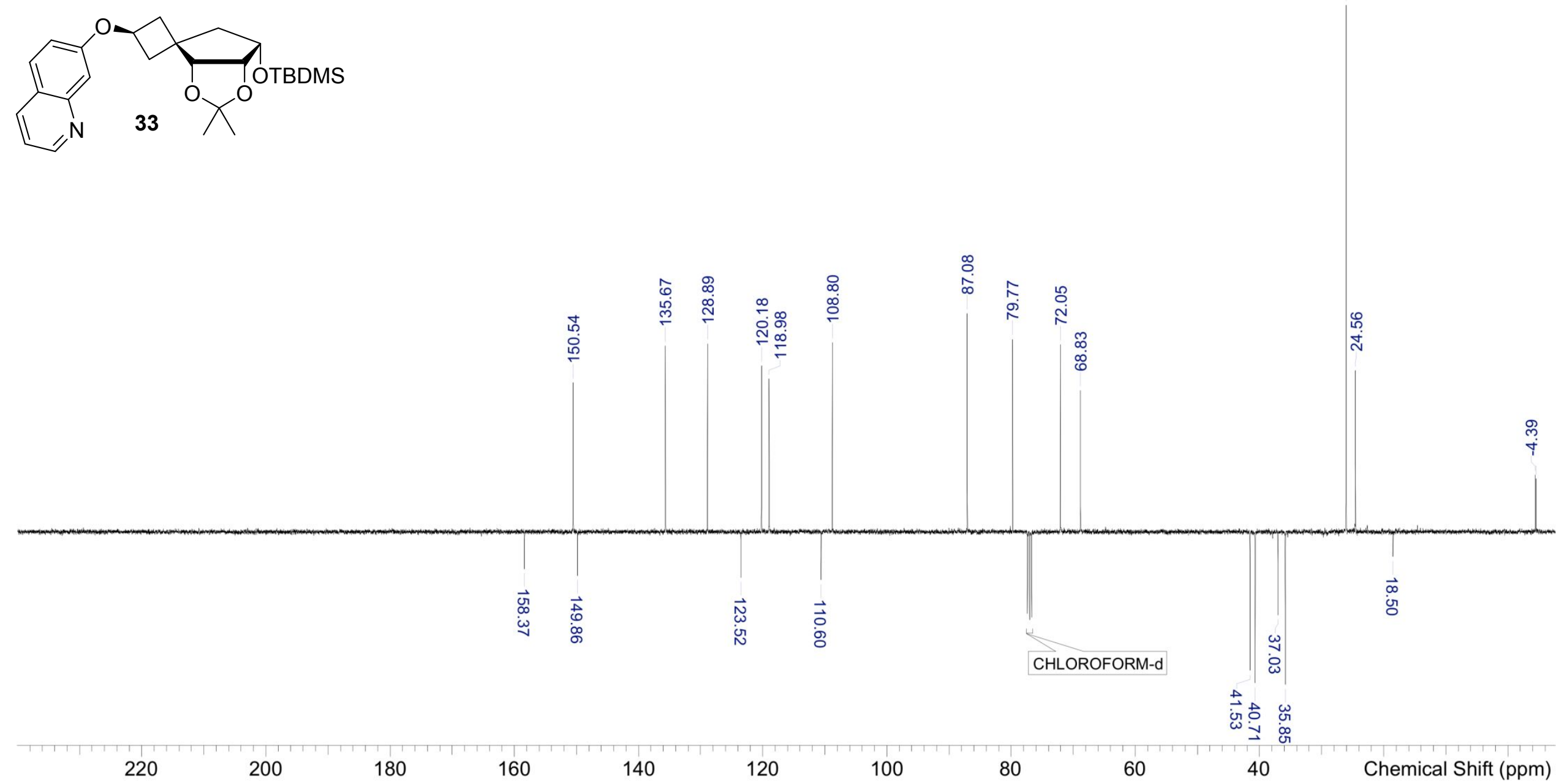


\section{SUPPORTING INFORMATION}

Frequency: $400 \mathrm{MHz}$
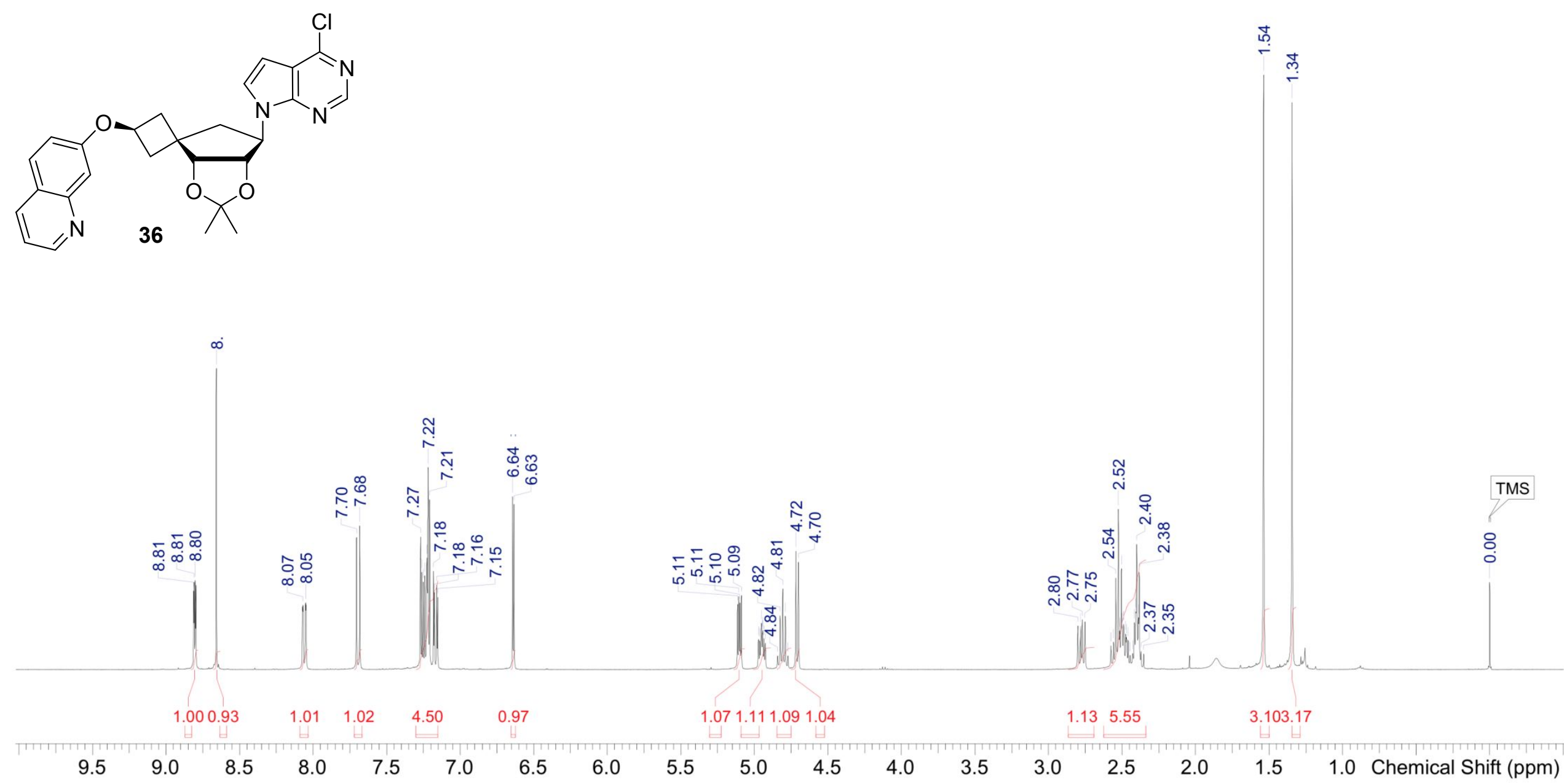


\section{SUPPORTING INFORMATION}

Frequency: $101 \mathrm{MHz}$
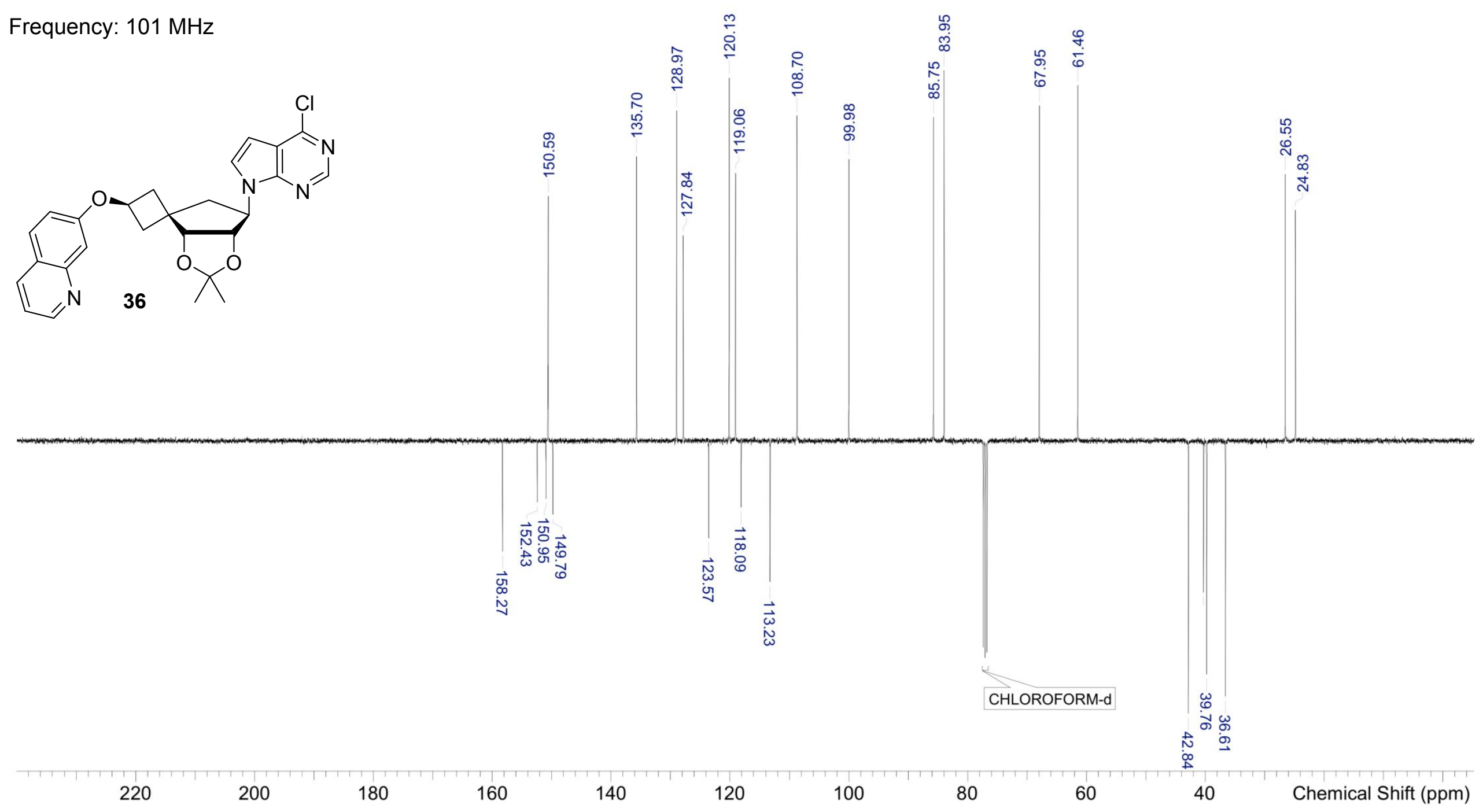


\section{SUPPORTING INFORMATION}

Frequency: $400 \mathrm{MHz}$
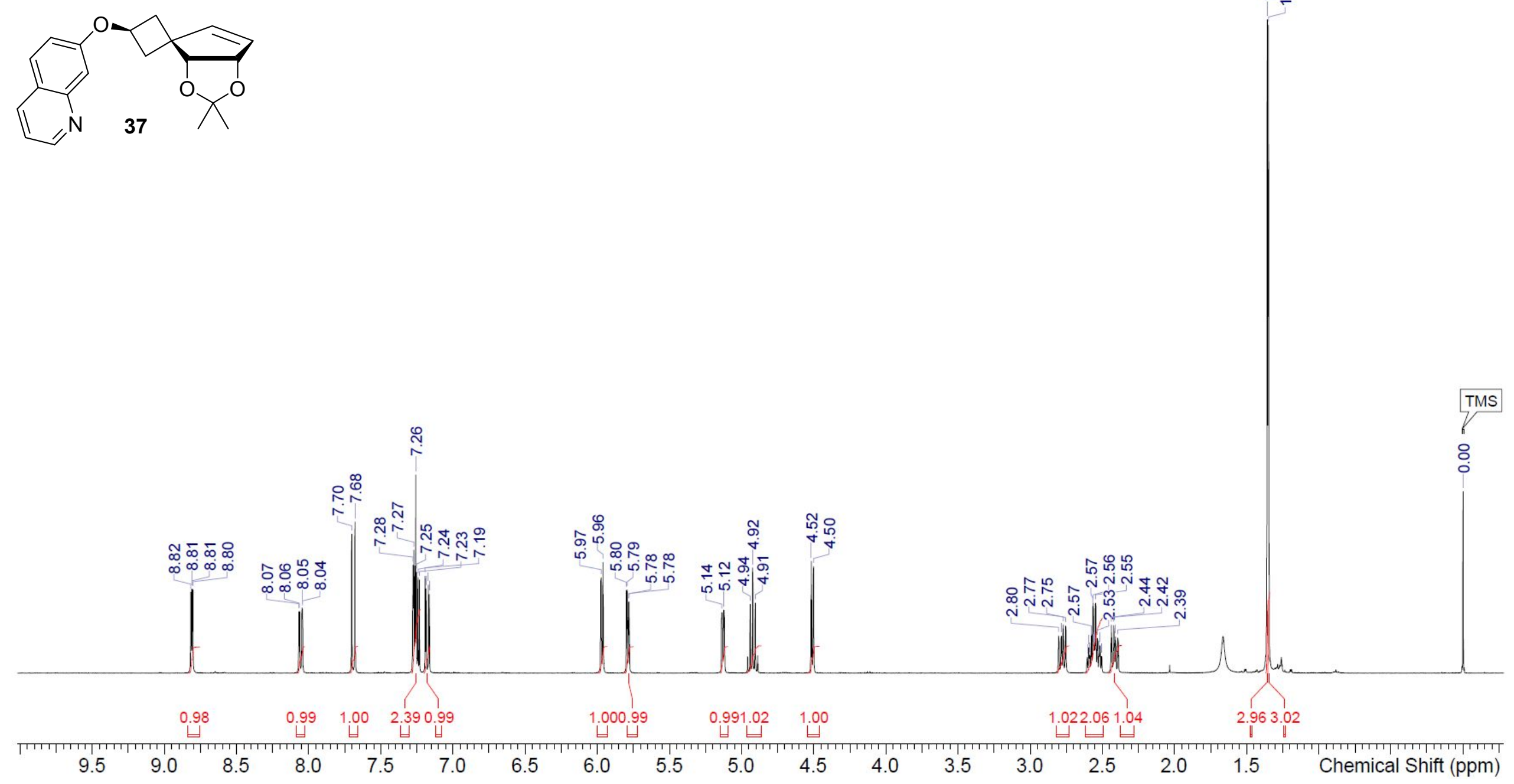


\section{SUPPORTING INFORMATION}

Frequency: $101 \mathrm{MHz}$

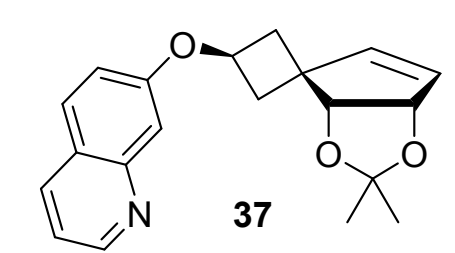

-

ํ.

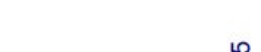

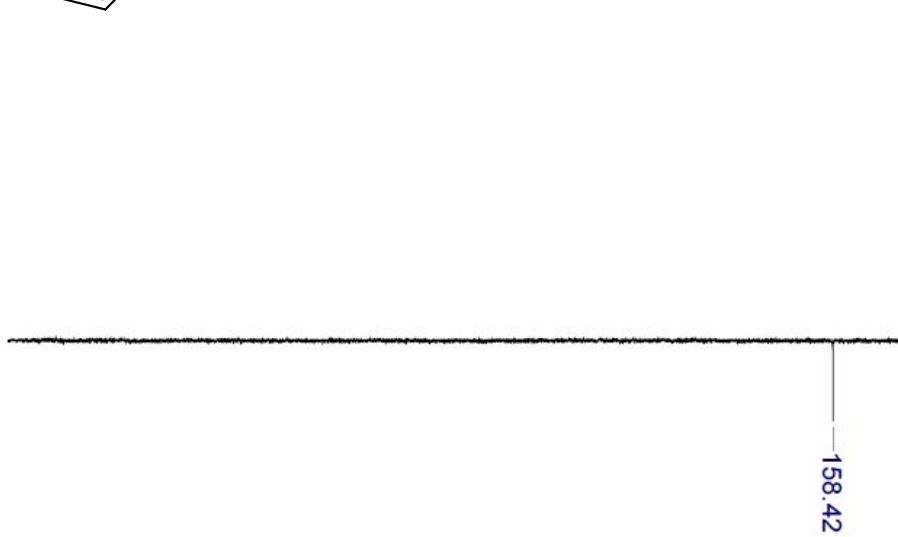

先

$\frac{0}{\infty}$

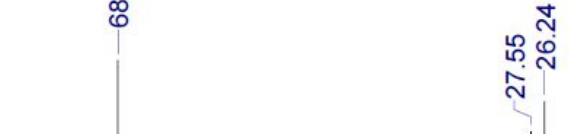

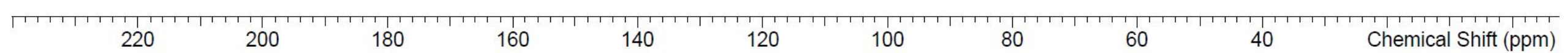




\section{SUPPORTING INFORMATION}

\section{Frequency: $400 \mathrm{MHz}$}
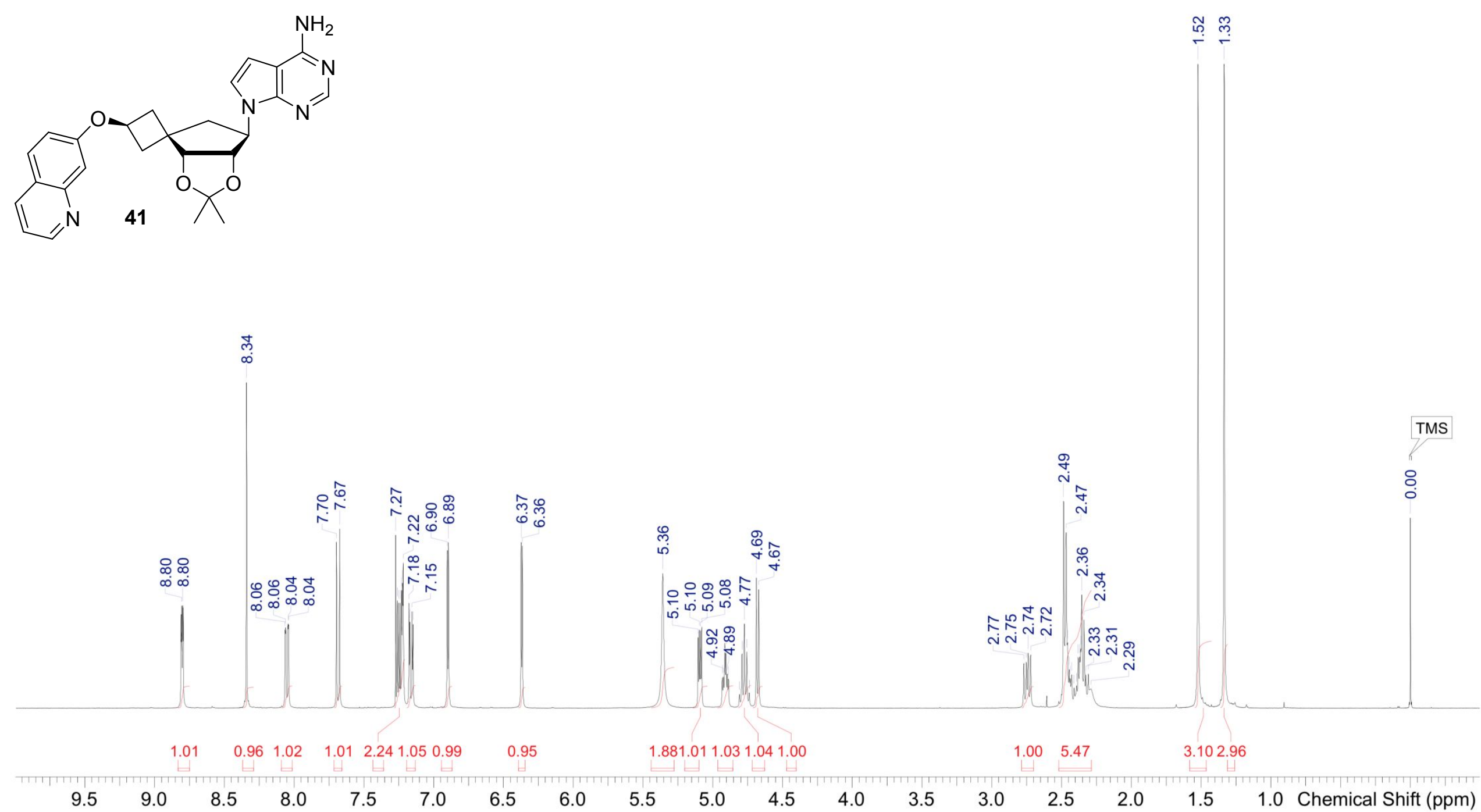


\section{SUPPORTING INFORMATION}

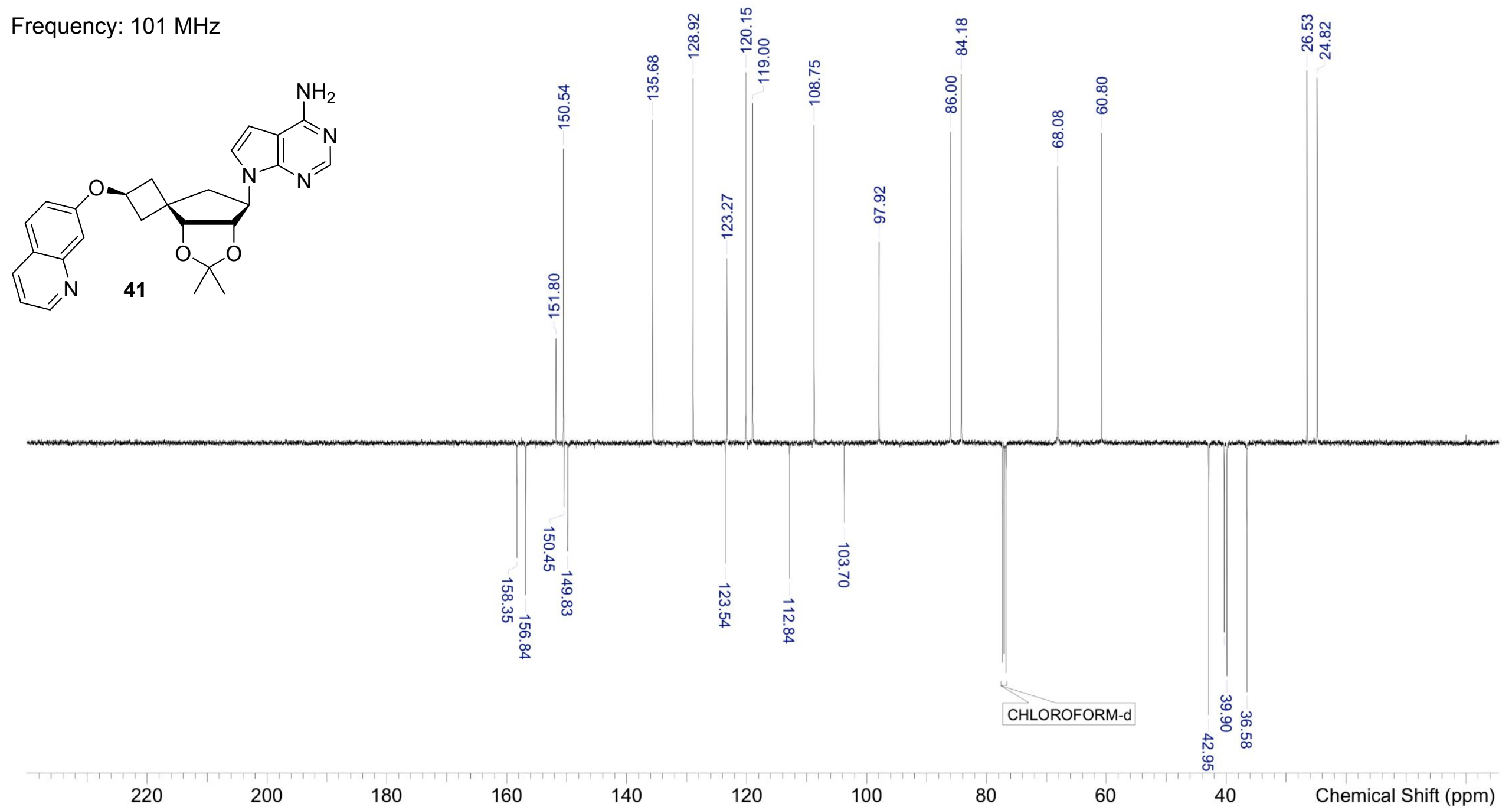




\section{SUPPORTING INFORMATION}

\section{Frequency: $400 \mathrm{MHz}$}
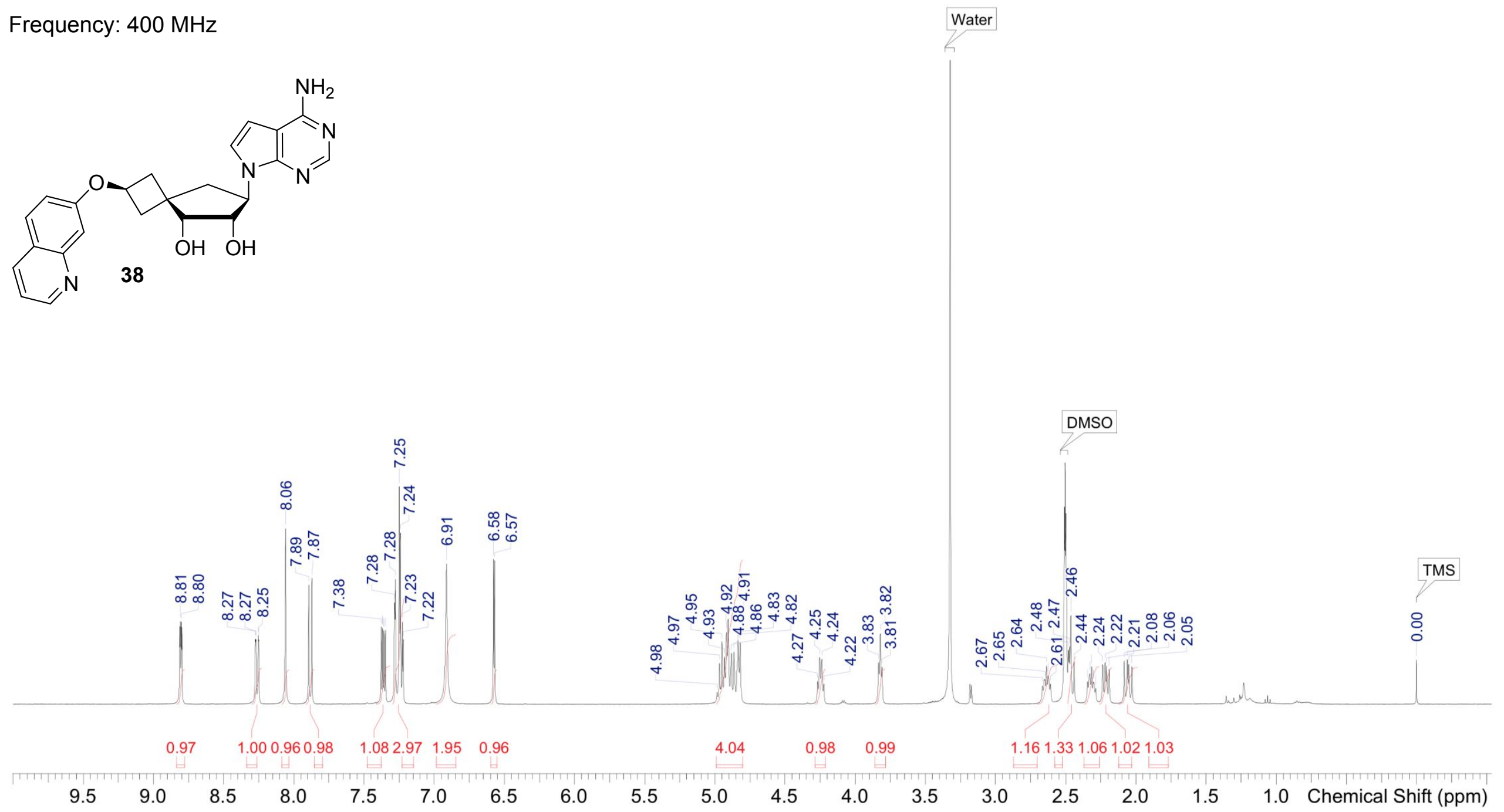


\section{SUPPORTING INFORMATION}

Frequency: $101 \mathrm{MHz}$
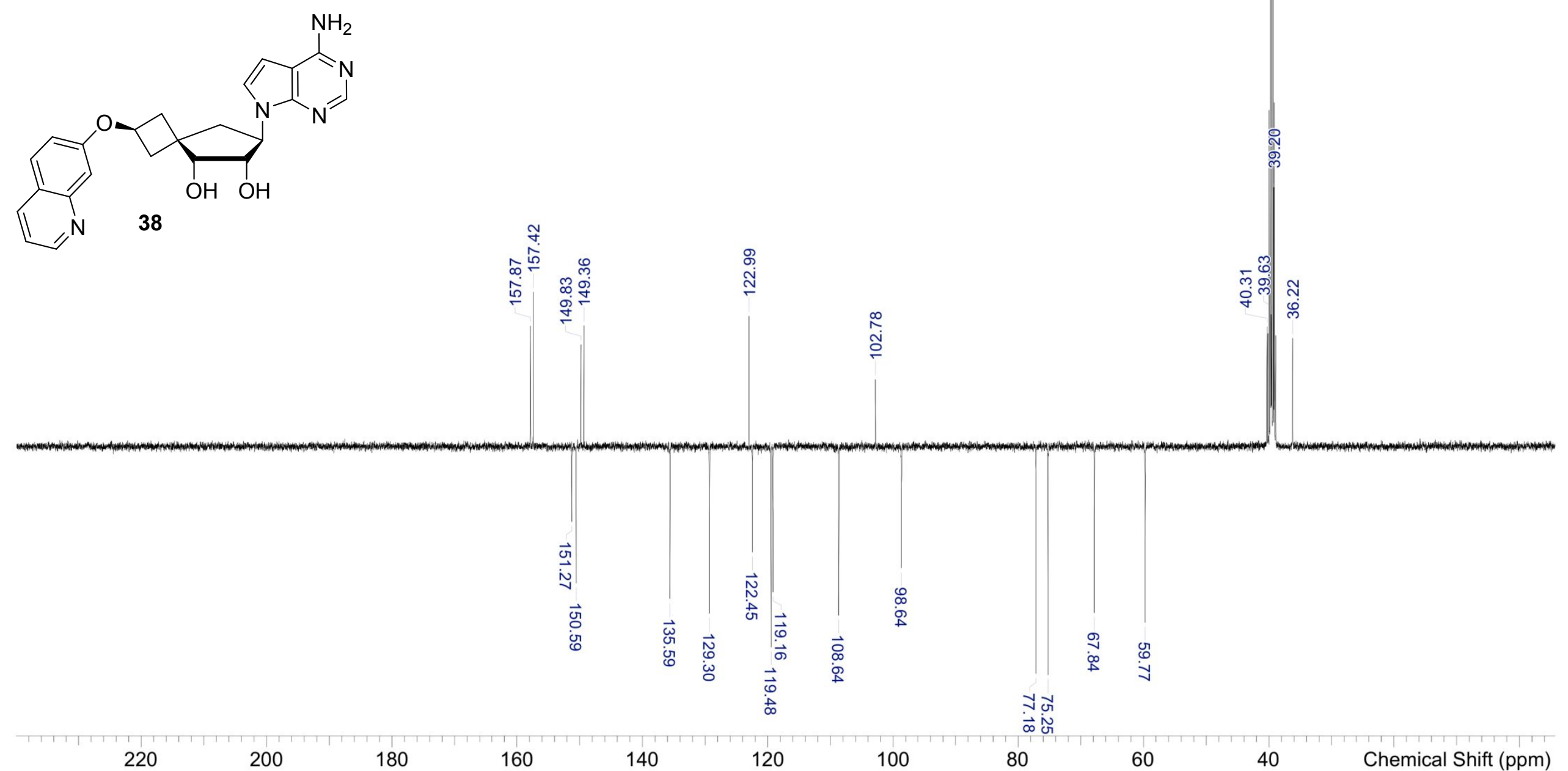


\section{SUPPORTING INFORMATION}

Frequency: $400 \mathrm{MHz}$
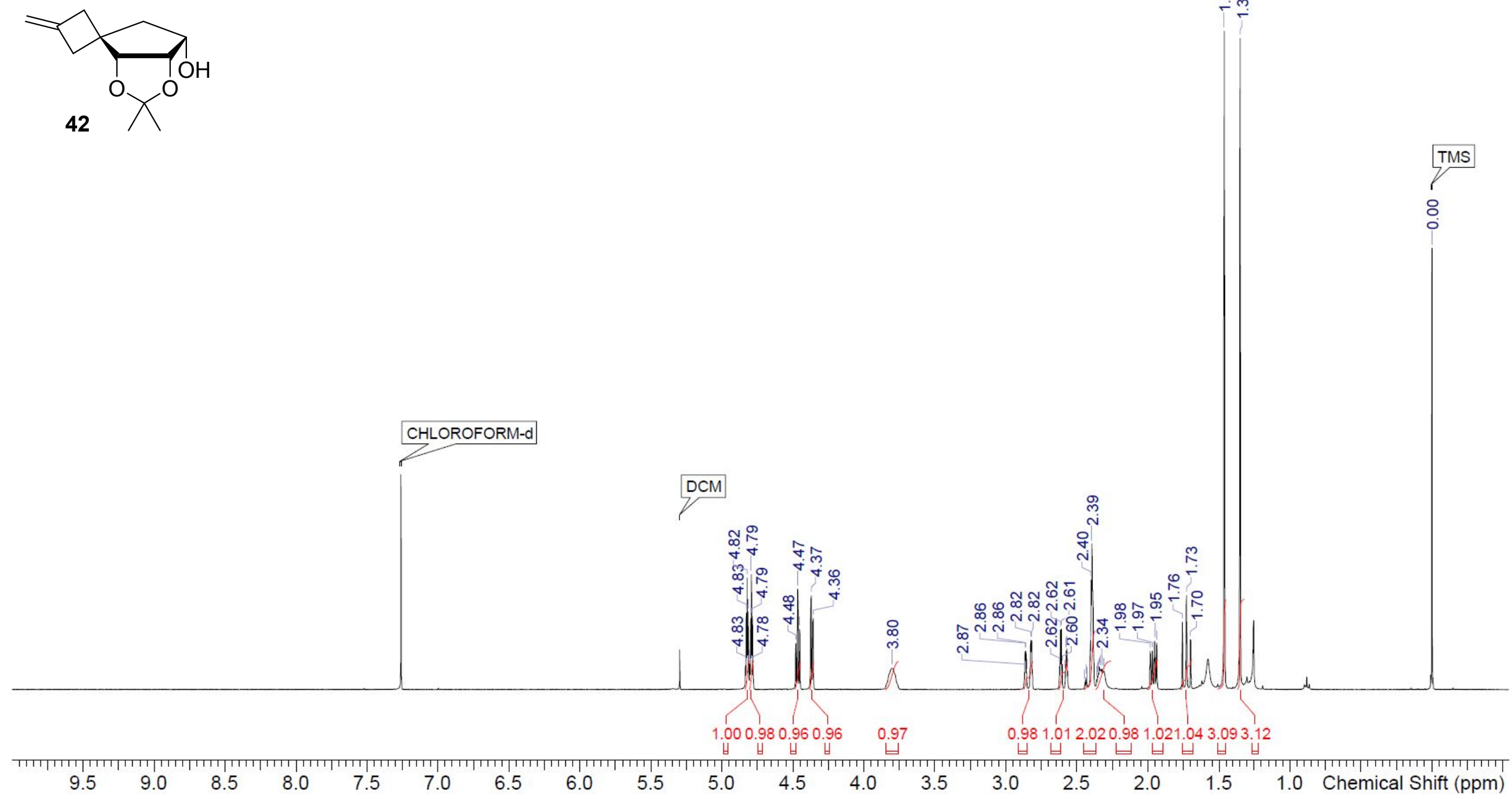


\section{SUPPORTING INFORMATION}

Frequency: $101 \mathrm{MHz}$
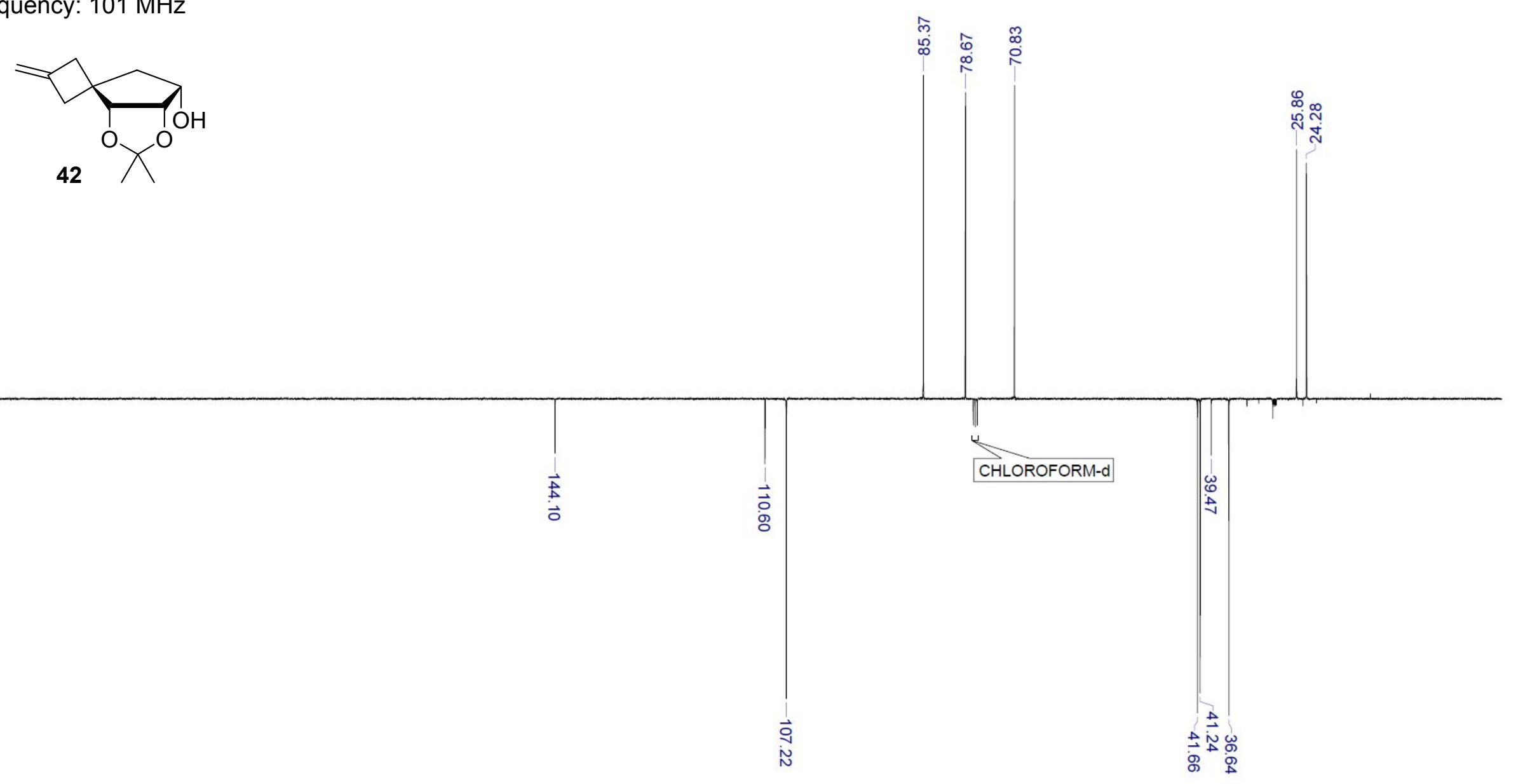


\section{SUPPORTING INFORMATION}

Frequency: $400 \mathrm{MHz}$
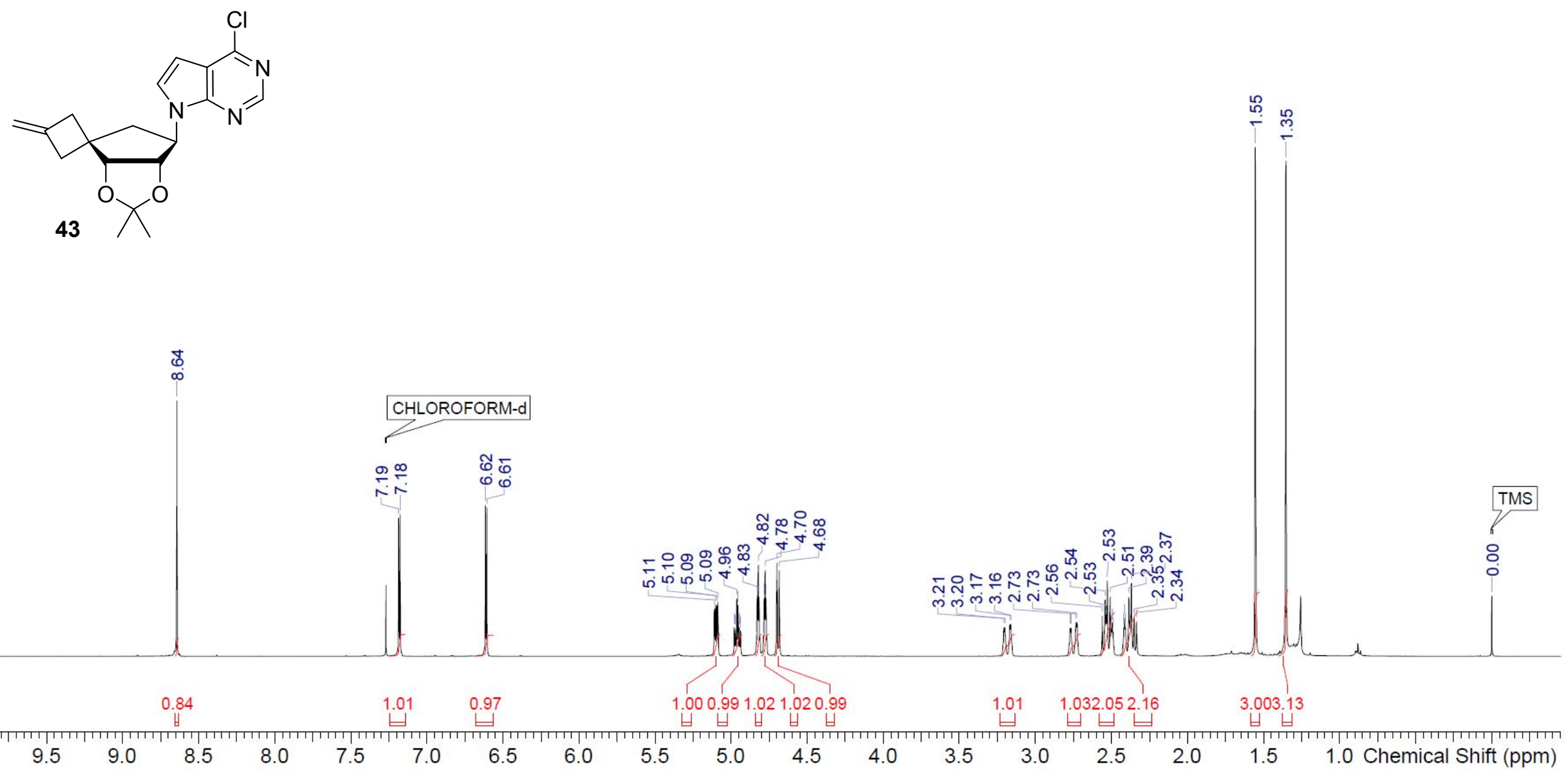


\section{SUPPORTING INFORMATION}

Frequency: $101 \mathrm{MHz}$
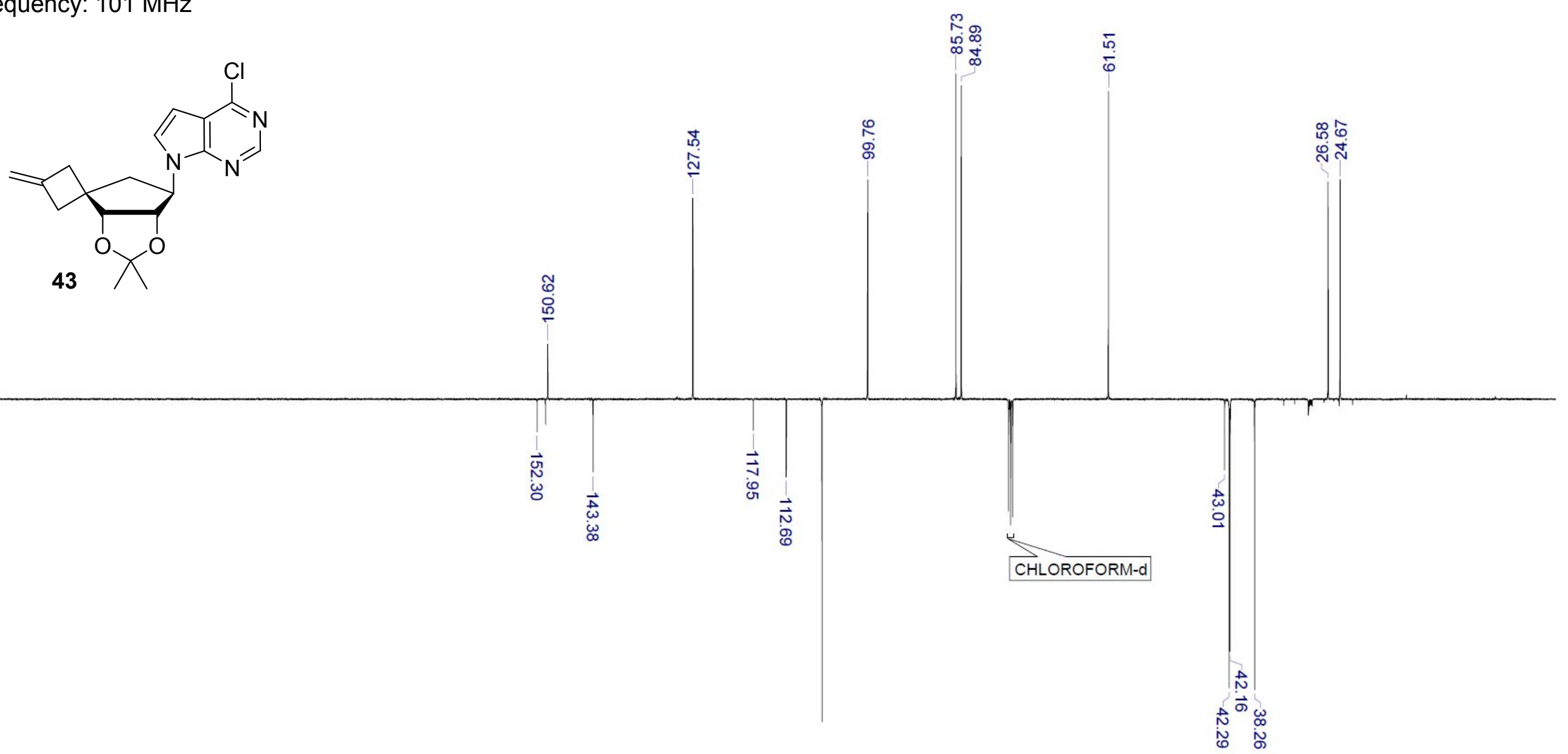


\section{SUPPORTING INFORMATION}

\section{Frequency: $400 \mathrm{MHz}$}

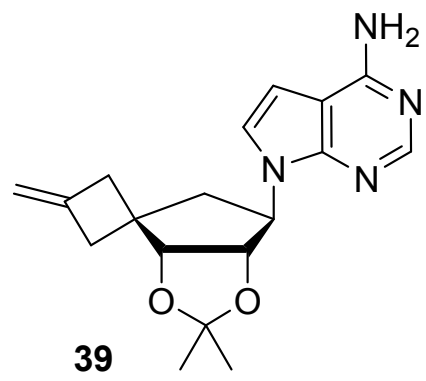

菅

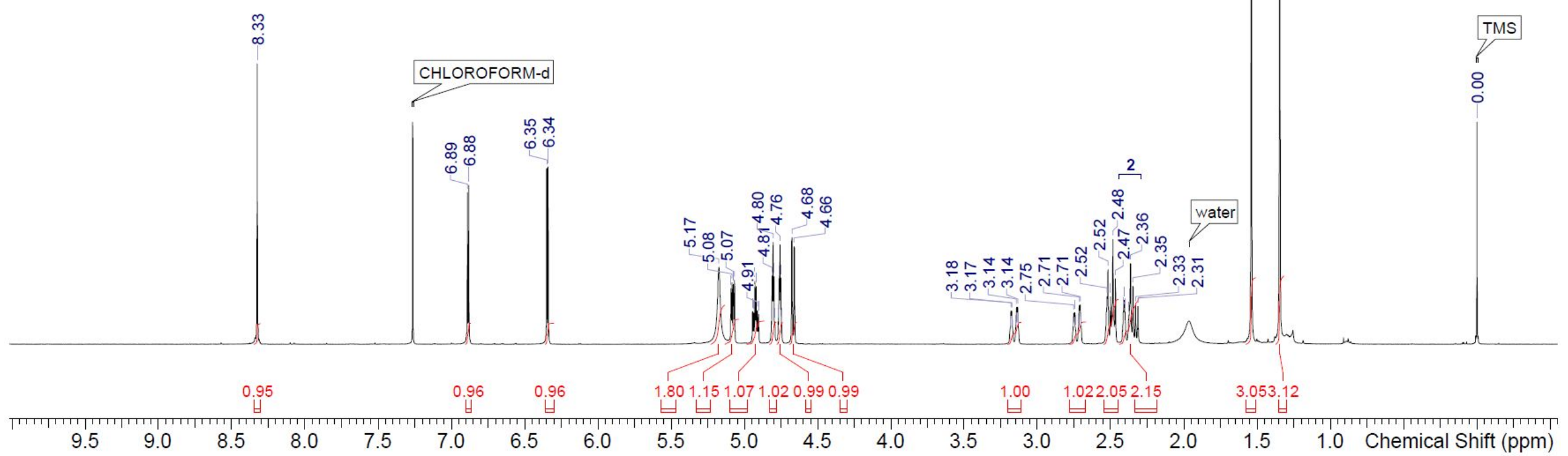




\section{SUPPORTING INFORMATION}

Frequency: $101 \mathrm{MHz}$

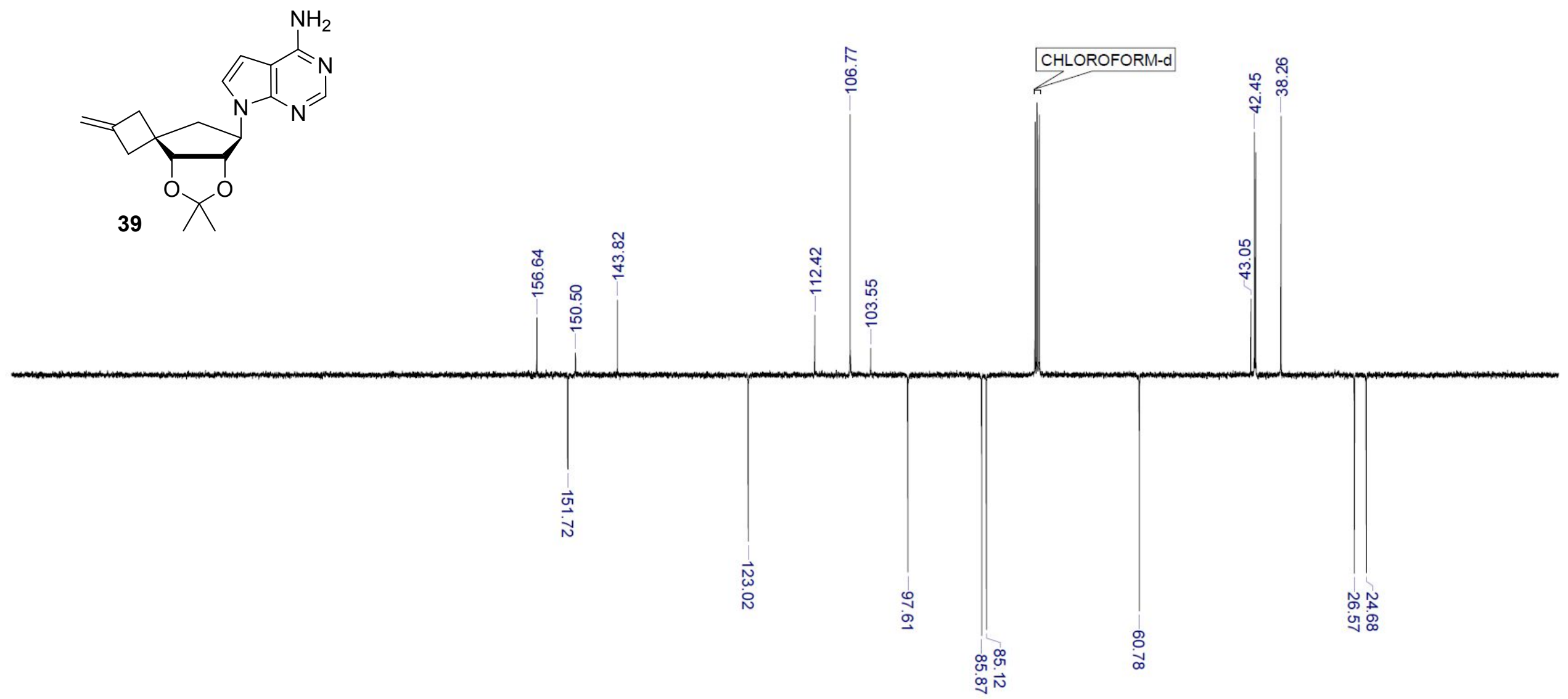




\section{SUPPORTING INFORMATION}

Frequency: $400 \mathrm{MHz}$
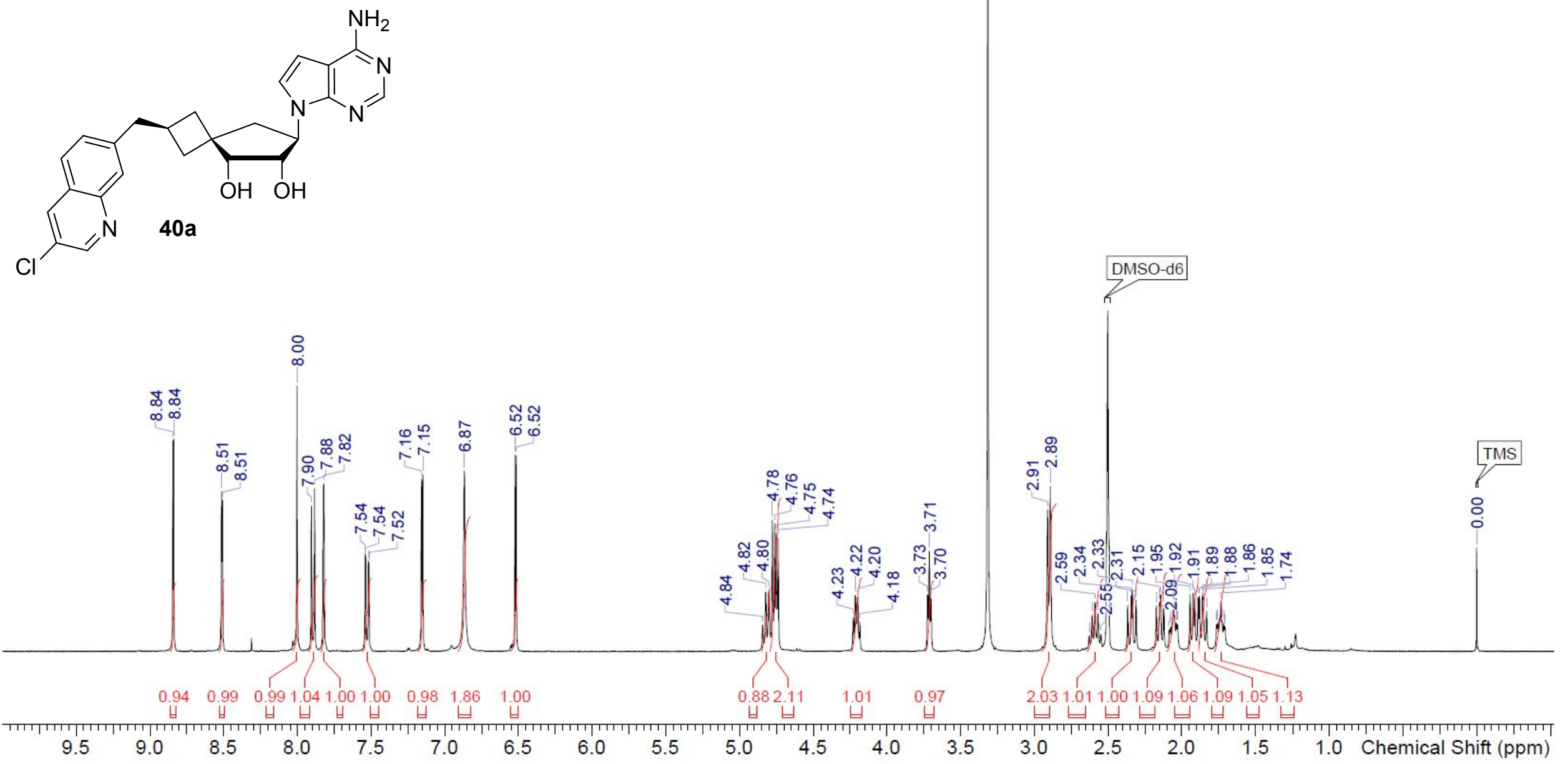


\section{SUPPORTING INFORMATION}

Frequency: $101 \mathrm{MHz}$
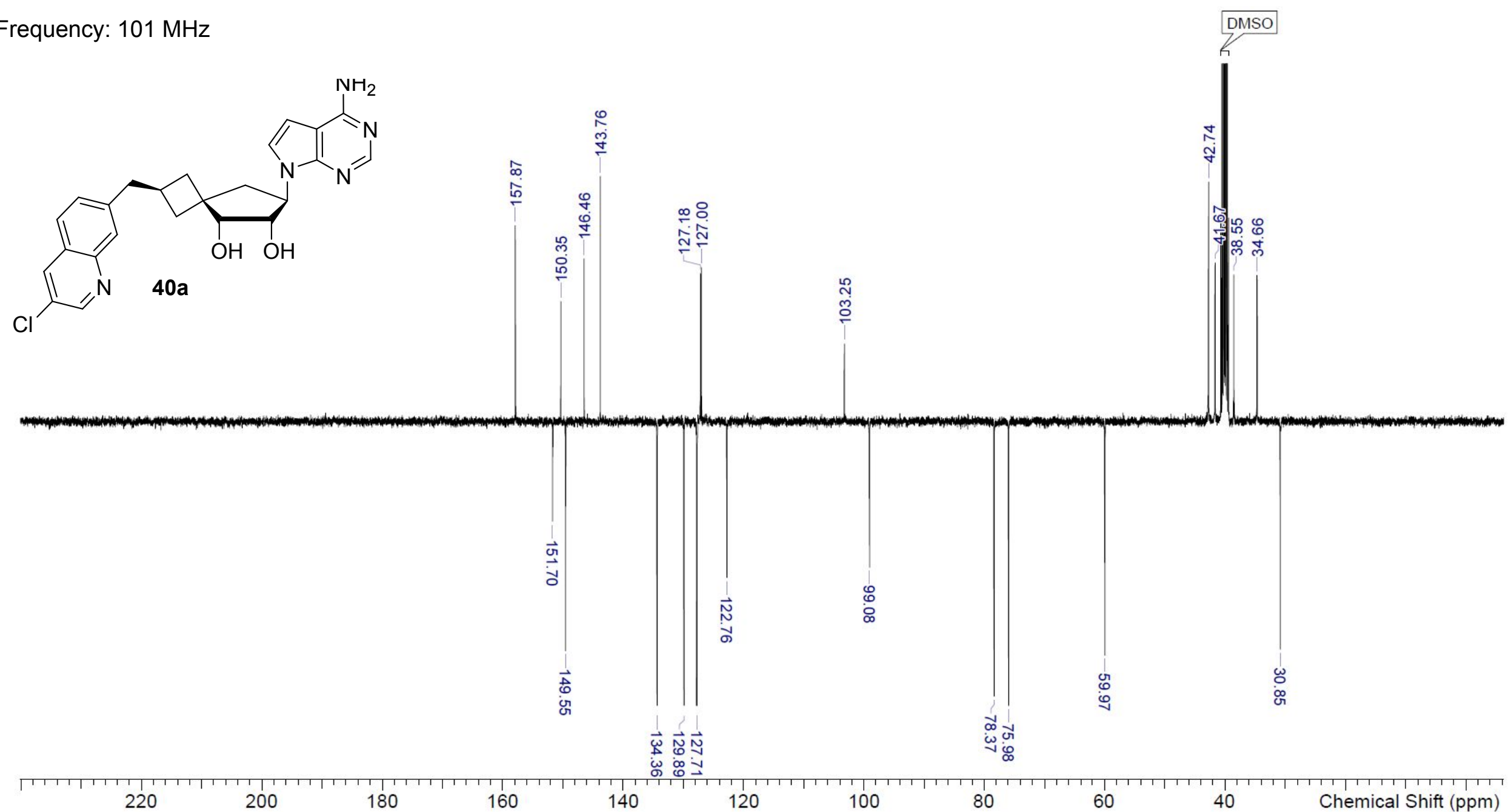


\section{SUPPORTING INFORMATION}

Frequency: $400 \mathrm{MHz}$
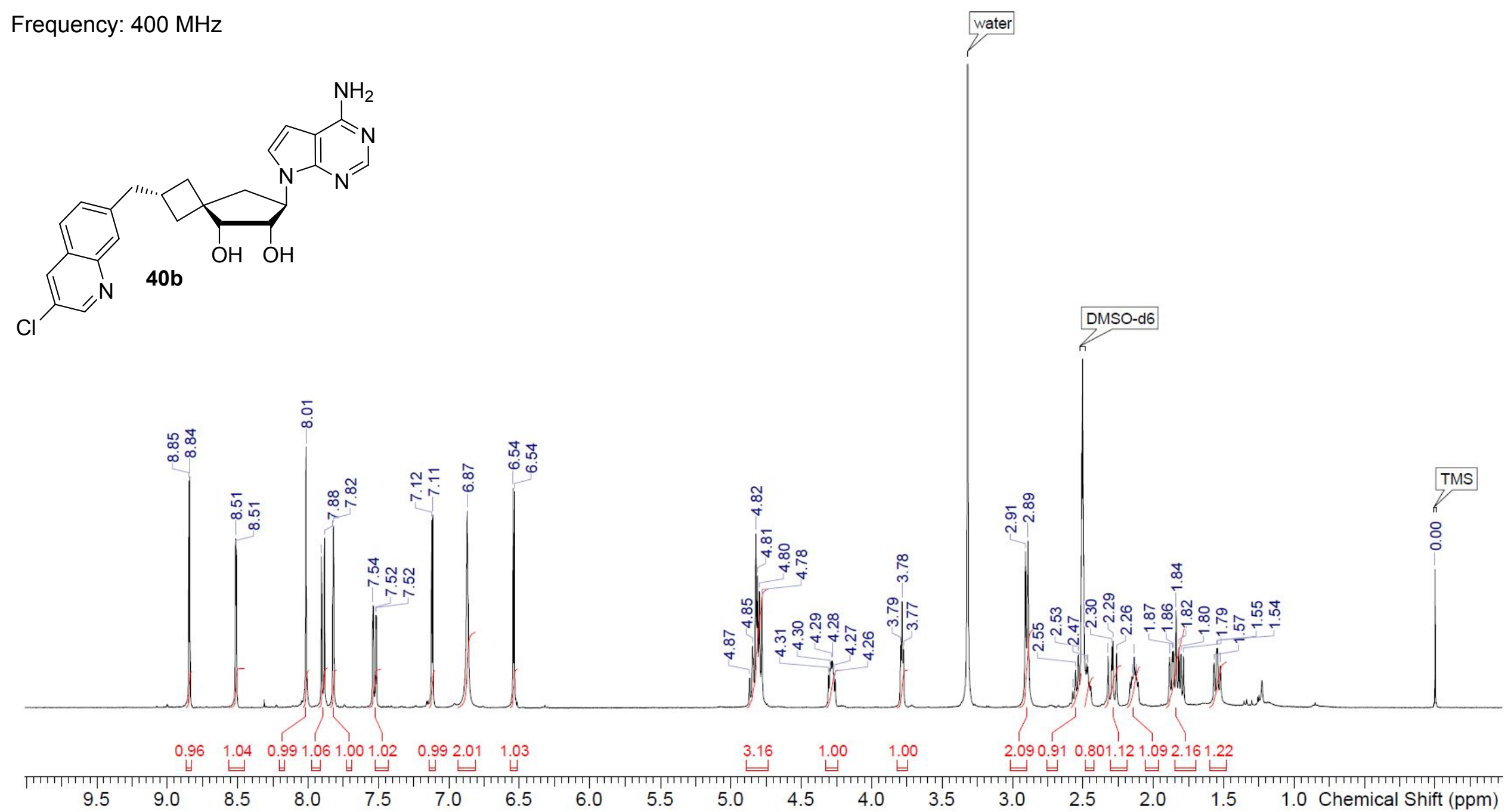


\section{SUPPORTING INFORMATION}

Frequency: $101 \mathrm{MHz}$
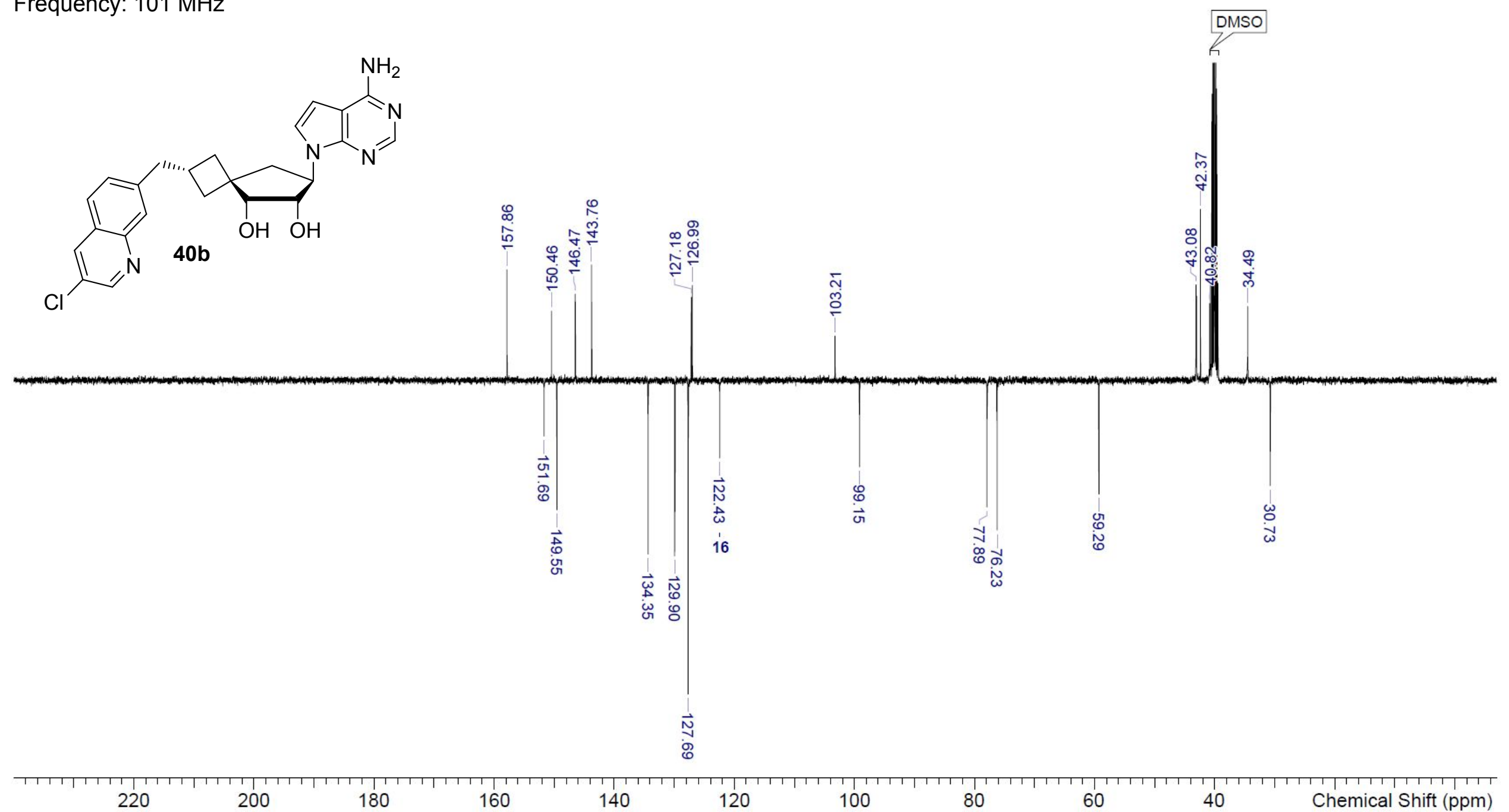
SUPPORTING INFORMATION

S101 